\title{
Scaling of internal wall temperatures in enclosure fires
}

Ying Zhen Li, Tommy Hertzberg

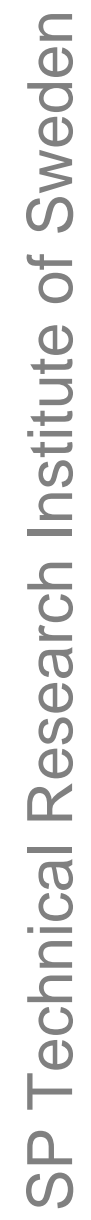

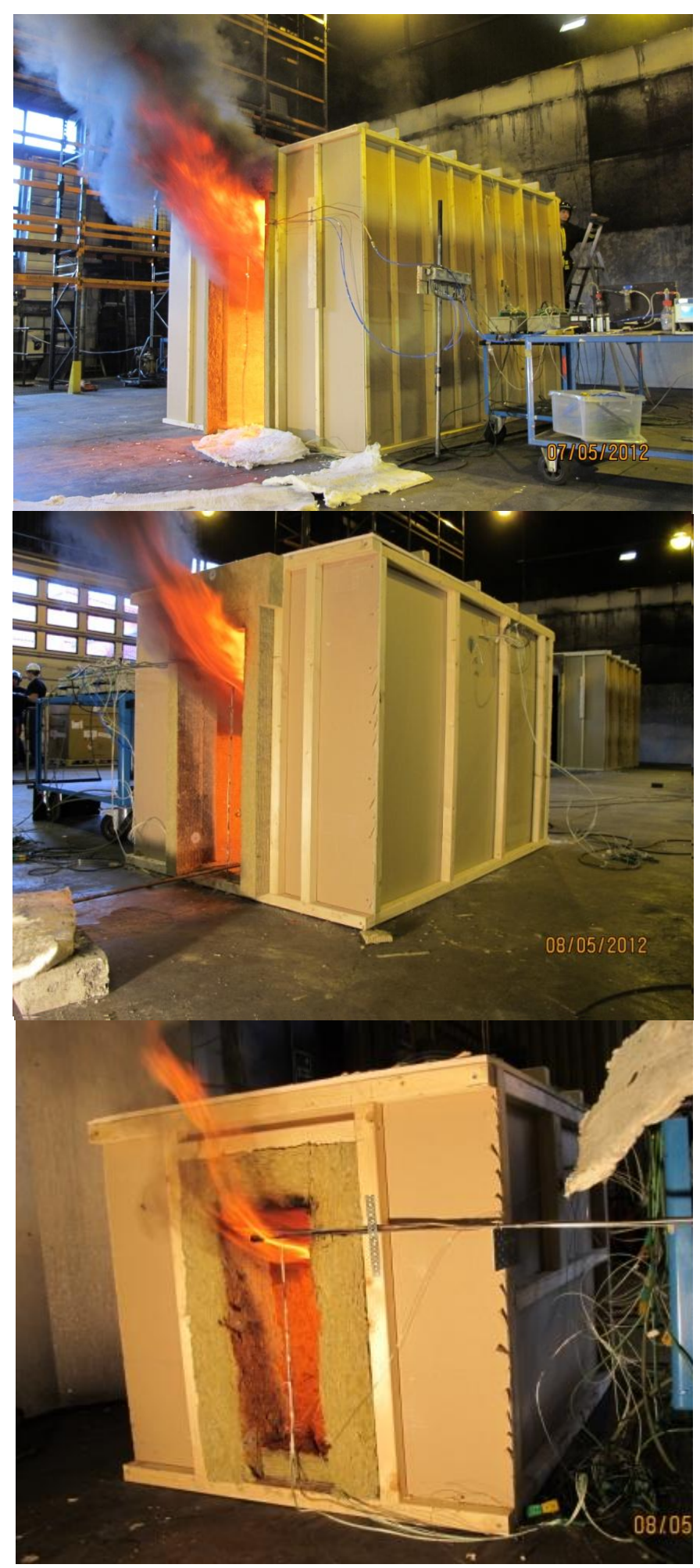


Scaling of internal wall temperatures in enclosure fires

Ying Zhen Li, Tommy Hertzberg 


\section{Abstract}

\section{Scaling of internal wall temperatures in enclosure fires}

The scaling of enclosure fires, including internal wall temperatures and heat fluxes, are thoroughly analyzed and a method of scaling internal wall temperatures is proposed. Two series of room fires were tested in three different scales, i.e. full scale (1:1), medium scale (1:2) and small scale (1:3.5), according to the theory. The fire source was either placed at the center or in the corner of the enclosures.

The measured internal wall temperatures, incident heat fluxes, gas temperatures, gas velocities and gas concentrations in different scales are compared and analyzed. The results show that the proposed scaling method is able to scale the internal wall temperature very well, especially in medium scale. The incident heat fluxes and gas velocities are also scaled very well. The gas temperatures and gas concentrations are scaled generally relatively well.

The calculation of upper layer gas temperatures in the compartment fires are investigated. The results show that both the maximum ceiling excess gas temperatures and average ceiling excess gas temperatures at the upper layer for corner fires are higher than those for center fires, and the ratio is 1.25 for both the maximum and the average ceiling excess gas temperatures. The maximum excess gas temperature normally exists right above the fire and is approximately $19 \%$ higher than the average values for both center fires and corner fires. The widely used $\mathrm{MQH}$ equation cannot directly be used to estimate the gas temperatures in compartments covered with insulating materials. A correlation has been proposed to estimate the upper layer gas temperatures in compartments covered by similar insulating materials for both center fires and corner fires. The equation is expected to be valid for gas temperatures below $700{ }^{\circ} \mathrm{C}$ or slightly higher, but not over $1000{ }^{\circ} \mathrm{C}$.

Key words: scaling; enclosure fire; internal wall temperature; wall material; gas temperature

SP Sveriges Tekniska Forskningsinstitut

SP Technical Research Institute of Sweden

SP Report 2013:12

ISBN 978-91-87017-96-4

ISSN 0284-5172

Borås 2013 


\section{Contents}

$\begin{array}{ll}\text { Abstract } & 3\end{array}$

$\begin{array}{lr}\text { Contents } & 4\end{array}$

$\begin{array}{ll}\text { Preface } & 5\end{array}$

$\begin{array}{lr}\text { Summary } & 6\end{array}$

$\begin{array}{ll}\text { Nomenclature } & 7\end{array}$

$\begin{array}{lll}1 & \text { Introduction } & 8\end{array}$

$2 \quad$ Scaling theory $\quad 10$

$3 \quad$ Test set-up 12

$\begin{array}{lll}3.1 & \text { Room geometry and structure } & 13\end{array}$

$\begin{array}{ll}3.2 & 13\end{array}$

$\begin{array}{lll}3.3 & \text { Fire sources } & 14\end{array}$

3.4 Measurements and instrumentation 14

$\begin{array}{llr}4 & \text { Test procedure } & 18\end{array}$

$5 \quad$ Summary of tests data $\quad 19$

5.1 Gas temperatures 19

$\begin{array}{lll}5.2 & 19\end{array}$

$\begin{array}{lll}5.3 & \text { Gas flow and concentration } & 19\end{array}$

$\begin{array}{lll}5.4 & \text { Temperatures inside the wall } & 19\end{array}$

$6 \quad$ Discussion of results $\quad 24$

$\begin{array}{lll}6.1 & \text { Scaling of gas temperature } & 24\end{array}$

$\begin{array}{ll}\text { 6.2 Scaling of gas flow and concentration } & 31\end{array}$

6.3 Scaling of incident heat flux 34

$\begin{array}{lll}6.4 & \text { Scaling of internal wall temperature } & 37\end{array}$

6.5 Calculation of ceiling gas temperatures 43

6.6 Applications in lightweight constructions 49

$7 \quad$ Conclusions $\quad 50$

$8 \quad$ References $\quad 52$

Appendix A - Scaling of internal wall temperatures 54

Appendix B - Layout of instrumentation in model scales 63

Appendix D - Test Results $\quad 76$ 


\section{Preface}

This project was sponsored by the SP Competence platform "New designs at sea", which is gratefully acknowledged.

Acknowledgement to our colleagues Michael Försth and Michael Rahm for their contribution to the test plan at the early stage of the project. Without them this project would not have been possible.

Thanks to Glenn Appel for his valuable assistance during the tests. The other personnel at SP Fire Technology, Michael Magnusson, Henrik Fredriksson, Sven-Gunnar Gustafsson and Emil Norberg are acknowledged for the construction of the test rig and the assistance during the performance of the tests. Thanks to Prof. Haukur Ingason and Dr Margaret Simonson McNamee for their valuable suggestions and comments. 


\section{Summary}

A total of 7 tests were carried out in a full scale room, a medium scale room and a small scale room. The main focus of these tests is scaling of internal wall temperatures inside the wall materials.

The scaling of enclosure fires, including internal wall temperatures and heat fluxes, is thoroughly analyzed and a method for scaling internal wall temperatures is proposed. Two series of room fire tests were carried out in three different scales, i.e. full scale (1:1), medium scale (1:2) and small scale (1:3.5), according to the theory. The fire source was either placed at the center or in the corner of the enclosures.

The measured internal wall temperatures in different scales of enclosure fires were compared and analyzed. The results show that the proposed scaling method is able to scale internal wall temperatures very well, especially in medium scale. The gas temperatures, gas concentrations, gas flows through the door and incident heat fluxes generally are also scaled well.

The gas temperatures at upper layer are also investigated. The results show that both maximum ceiling excess gas temperatures and average ceiling excess gas temperatures for corner fires are higher than those for center fires, and the ratio is 1.25 for both the maximum and the average ceiling excess gas temperatures. The maximum excess gas temperature normally exists right above the fire and is approximately $19 \%$ higher than the average values for both center fires and corner fires. For center fires, the widely used $\mathrm{MQH}$ equation can be used to estimate the maximum ceiling gas temperatures, but it significantly overestimates the average gas temperatures. For corner fires, the MQH equation can be used to approximately estimate the average ceiling gas temperatures, but it significantly underestimates the maximum ceiling gas temperatures. An correlation has been proposed to estimate the upper layer gas temperatures in compartments covered by similar insulating materials for both center fires and corner fires. The equation is expected to be valid for gas temperatures below $700{ }^{\circ} \mathrm{C}$ or slightly higher, but not over $1000{ }^{\circ} \mathrm{C}$. The proposed equation could only be used for estimation of upper layer gas temperatures in compartments covered by similar insulating materials. 


\section{Nomenclature}

\begin{tabular}{|c|c|c|c|}
\hline$a$ & thermal diffusivity $\left(\mathrm{m}^{2} / \mathrm{s}\right)$ & \multicolumn{2}{|c|}{ Greek symbols } \\
\hline$A$ & $\operatorname{area}\left(\mathrm{m}^{2}\right)$ & $\rho$ & gas density $\left(\mathrm{kg} / \mathrm{m}^{3}\right)$ \\
\hline$A_{b}$ & bounding area of hot gases $\left(\mathrm{m}^{2}\right)$ & $\beta$ & expansion coefficinet $(1 / \mathrm{K})$ \\
\hline$c$ & heat capacity $(\mathrm{kJ} / \mathrm{kg} \cdot \mathrm{K})$ & $\delta$ & physical or thermal thickness (m) \\
\hline$C_{d}$ & flow coefficient & $\varepsilon$ & emissivity \\
\hline$C_{\text {heat }, \beta}$ & lumped heat capacity $(\mathrm{kJ} / \mathrm{kg} \cdot \mathrm{K})$ & $\sigma$ & Stefan-Boltzmann constant \\
\hline $\begin{array}{l}=1 / 3 \\
C_{2}\end{array}$ & coefficient & & $\begin{array}{l}\left(\mathrm{kW} / \mathrm{m}^{2} \cdot \mathrm{K}^{4}\right) \\
\text { absorption coefficient }(1 / \mathrm{m})\end{array}$ \\
\hline$D$ & burner diameter (m) & & ratio of the effective radiation \\
\hline E & energy content $(\mathrm{kJ})$ & & area to the area of opening \\
\hline$g$ & gravity acceleration $(\mathrm{m} 2 / \mathrm{s})$ & & \\
\hline$h$ & heat transfer coefficient $\left(\mathrm{kJ} / \mathrm{m}^{2} \cdot \mathrm{K}\right)$ & Sup & and subscript \\
\hline$H$ & height of an opening (m) & & ambient \\
\hline$\Delta H_{c}$ & heat of combustion $(\mathrm{kJ} / \mathrm{kg})$ & & bounding \\
\hline$k$ & thermal conductivity $(\mathrm{kW} / \mathrm{m} \cdot \mathrm{K})$ & $b w$ & backside of the wall \\
\hline$K_{\text {cond }}$ & Correction factor $(\mathrm{kW} / \mathrm{m} \cdot \mathrm{K})$ & $c$ & convection \\
\hline$l$ & length scale $(\mathrm{m})$ & $f$ & Fuel \\
\hline$L_{f}$ & flame length (m) & & full scale \\
\hline$L_{m}$ & mean beam length $(\mathrm{m})$ & & Gas \\
\hline$m$ & fuel mass $(\mathrm{kg})$ & & ith step \\
\hline$\dot{m}_{g}^{\prime \prime}$ & mass flow rate $\left(\mathrm{kg} / \mathrm{m}^{2} \cdot \mathrm{s}\right)$ & inc & incident heat flux \\
\hline $\mathrm{Nu}$ & Nusselt Number & & conduction \\
\hline$P$ & pressure $(\mathrm{Pa})$ & & mean \\
\hline$\Delta P$ & pressure difference $(\mathrm{Pa})$ & & model scale \\
\hline $\operatorname{Pr}$ & Prandtl number & net & net value \\
\hline$Q$ & heat release rate $(\mathrm{kW})$ & & opening or reference \\
\hline$\dot{q}^{\prime \prime}$ & heat flux $\left(\mathrm{kW} / \mathrm{m}^{2}\right)$ & $P T$ & Plate thermometer \\
\hline$R$ & heat resistance $\left(\mathrm{m}^{2} \cdot \mathrm{K} / \mathrm{kJ}\right)$ & & radiation \\
\hline $\bar{R}$ & lumped resistance $\left(\mathrm{m}^{2} \cdot \mathrm{K} / \mathrm{kJ}\right)$ & $s$ & Solid \\
\hline $\mathrm{Ra}_{l}$ & Rayleigh number & $t$ & Total \\
\hline $\mathrm{Re}$ & Reynold Number & $w$ & Wall \\
\hline$t$ & time $(\mathrm{s})$ & & \\
\hline$T$ & temperature $(\mathrm{K})$ & & Abbreviations \\
\hline$\Delta T$ & excess temperature $(\mathrm{K})$ & $\mathrm{BP}$ & bi-directional probe \\
\hline$u$ & velocity $(\mathrm{m} / \mathrm{s})$ & & gas anlaysis \\
\hline$v$ & kinematic viscosity $\left(\mathrm{m}^{2} / \mathrm{s}\right)$ & PT & plate thermometer \\
\hline$V$ & volume $\left(\mathrm{m}^{3} / \mathrm{s}\right)$ & $\mathrm{TP}$ & thermocouple pile \\
\hline$V_{b}$ & volume of the hot gases $\left(\mathrm{m}^{3}\right)$ & $\mathrm{TS}$ & thermocouple series \\
\hline$V_{i s}$ & visibility $(\mathrm{m})$ & & \\
\hline$V_{t}$ & total volume of fuels $\left(\mathrm{m}^{3}\right)$ & & \\
\hline$Y$ & gas concentration $(\mathrm{kg} / \mathrm{kg})$ & & \\
\hline$z$ & depth below surface (m) & & \\
\hline
\end{tabular}




\section{Introduction}

Due to the complexity of the fire phenomenon, full scale tests are still the main and the most credible tools while investigating any new fire related phenomenon or any new fire protection system. The full scale tests provide the most valuable data, however, are also very costly, especially in some scenarios at a huge scale. Model scale tests are therefore a tempting alternative to the full scale tests.

In the past few decades, the physical scaling method has been widely used in fire community. Its application nearly covers every field of fire research, from free plume to fire suppression in tunnel fires. Despite its simplifications in applications, the scaling technique has significantly improved our understanding of fire dynamics. Heskestad [1] reviewed the scaling techniques mainly including the pressure modeling and the Froude modeling that were used in the fire community. Quintiere [2] also reviewed the scaling applications in fire research on ceiling jets, burning rate, flame spread and enclosure fires. Perricone et al. [3] investigated the thermal response of steel tube covered by insulating materials, however, the scaling laws used for the thick insulating materials could be questioned. Cross and Xin [4] examined the scaling of wood crib fires and found good agreement between the different scales. Scaling of water-based fire suppression systems has also been conducted in open and enclosure fires. Heskestad [5,6] carried out a series of gas and pool fire suppression tests to investigate the credibility of the scaling of the interaction of water sprays and flames, and obtained a simple correlation for extinguishment of gas and pool fires using water sprays. Quintiere et al's work [7] shows that the scaling of pool and gas fires works well, however, the comparison of results in rack-storage fires between model and full scale doesn't show good correlation. Yu et al. [8-10] tested and investigated the scaling of suppression of gas fires and pool fires using water mist systems and obtained good agreement between model scale and full scale.

SP Fire Technology has carried out extensive model scale tunnel fire tests in the past decade. Ingason and Li investigated the key fire parameters and smoke control in model scale tunnels with longitudinal ventilation [11] and with point extraction ventilation [12]. Ingason [13] also carried out a series of 1:10 model scale railcar tunnel fire tests to investigate the effect of openings on the fire sizes. Li et al. [14-21] carried out several series of model scale tunnel fire tests to investigate the critical velocity, backlayering length, maximum ceiling gas temperature, smoke control in cross-passages, and smoke control in rescue stations in long railway tunnels. Lönnermark et al. [22] carried out a 1:3 model scale metrocar fire tests for the full scale fire tests in Brunsberg tunnel [23]. Ingason [24] tested the water spray system in tunnel fires using hollow cone nozzles and wood crib fires. Deluge system and water curtain system were also tested. Li and Ingason [25] investigated the automatic water spray system in tunnel fires using full cone nozzles and wood crib fires. Response time of individual sprinklers was modeled by a scaling theory.

Despite much work on the scaling of fires in the open and the enclosures, the credibility of scaling of the internal wall temperatures has not been explored. The internal wall temperatures have attracted our special attention partly due to the relation to fire resistance of the walls. For example, in case of a fire in a lightweight construction, the temperature at the load-bearing structure should not exceed a certain value, e.g. $140{ }^{\circ} \mathrm{C}$, or else the structure will lose strength and collapse [26,27]. Further, the scaling of wall heat fluxes affects the gas temperatures in the enclosures.

Therefore, the scaling of internal wall temperatures needs to be investigated carefully in order to be able to trust experimental data from scaled enclosure fires, which is the main focus of this project. 
In this report, the scaling of enclosure fires including internal wall temperatures and heat fluxes are thoroughly analyzed and a method for scaling of internal wall temperatures is proposed. According to the theory, compartment fire tests were carried out in different scales. Based on the tests data, the scaling of internal wall temperatures, incident heat fluxes, gas temperatures, gas velocities and gas concentrations are analyzed. Further, the calculation of upper layer gas temperatures in the compartment fires are investigated. 


\section{$2 \quad$ Scaling theory}

The widely used and well known Froude scaling technique has been applied in this project. Although it is impossible and in most cases not necessary to preserve all the terms obtained by scaling theory simultaneously, the terms that are most important and most related to the study are preserved.

The Froude scaling has been used widely in enclosure fire research and results from model scale tests could fit large scale data well, see references [2,5]. A large amount of model scale tunnel fire tests carried out at SP also show that there is good agreement between model scale and large scale test results on many focused issues [11-21,28].

However, in most of the model scale tests that have been carried out worldwide, the thermal properties of the involved material were not taken into account. One reason could be that for those studies the internal wall temperatures were not the focus, however, the wall materials have strong influence on the scaling of the fires, especially at small scale.

In this project, a theoretical scaling study on the heat conduction inside the walls and heat fluxes has been conducted, see Appendix A. The scaling laws for internal wall temperatures are proposed and are investigated in the following based on the data obtained from two series of fire tests carried out in different scales of enclosures.

The rooms were built in a scale of 1:1, 1:2 and 1:3.5 respectively, which means that the sizes of the rooms are scaled geometrically according to the scaling ratios. The scaling of the key parameters is presented in the following. The detailed information about scaling of heat fluxes and internal wall temperatures can be found in Appendix A.

It is shown in Table 1 that gas temperatures and internal wall temperatures should be the same in all scales. However, it should be kept in mind that the correlations shown in Table 1 only correspond to the perfect scaling. In reality, there are always some parameters that cannot be scaled well and thus induce errors to the other related parameters. The key error in this type of scaling is produced by the scaling of heat fluxes.

Note that the scaling of these parameters is obtained based on the assumption of the same heat of combustion. If different fuels are used in model scale, some parameters will not be scaled as shown in Table 1.

There are two source terms in the controlling equations for the mass transfer and heat transfer in an enclosure fire, that is, the heat release rate and the mass loss rate, respectively. If we focus on the scaling of the mass loss rate, the gas concentration can still not be scaled well due to the failure of scaling of the buoyancy force and gas temperature. In such cases, the only solution is to focus on the scaling of the heat release rate, regardless of the species production. As a consequence, the heat release rate, energy content, velocity, time, temperature, and pressure will approximately scale as shown in Table 1. However, the overall gas concentration cannot scale well, and according to the scaling of the energy content, the fuel mass will scale as:

$$
\frac{m_{M}}{m_{F}}=\left(\frac{\Delta H_{c, F}}{\Delta H_{c, M}}\right)\left(\frac{l_{M}}{l_{F}}\right)^{5 / 2}
$$

It should be kept in mind that different fuel types may affect the heat radiation due to the difference in soot yield and species production. 
Table 1 A list of scaling correlations for the model tunnel.

\begin{tabular}{|l|c|c|}
\hline \multicolumn{1}{|c|}{ Type of unit } & Scaling & $\begin{array}{c}\text { Equation } \\
\text { number }\end{array}$ \\
\hline Heat Release Rate (HRR) $(\mathrm{kW})$ & $Q_{M} / Q_{F}=\left(l_{M} / l_{F}\right)^{5 / 2}$ & $(2)$ \\
\hline Velocity $(\mathrm{m} / \mathrm{s})$ & $u_{M} / u_{F}=\left(l_{M} / l_{F}\right)^{1 / 2}$ & $(3)$ \\
\hline Time $(\mathrm{s})$ & $t_{M} / t_{F}=\left(l_{M} / l_{F}\right)^{1 / 2}$ & $(5)$ \\
\hline Energy $(\mathrm{kJ})$ & $E_{M} / E_{F}=\left(l_{M} / l_{F}\right)^{3}$ & $(6)$ \\
\hline Temperature $(\mathrm{K})$ & $T_{F} / T_{M}=1$ & $(7)$ \\
\hline Pressure $(\mathrm{Pa})$ & $P_{M} / P_{F}=\left(l_{F} / l_{M}\right)^{1}$ & $(8)$ \\
\hline heat flux $(\mathrm{kW})$ & $\dot{q}_{M}^{\prime \prime} / \dot{q}_{F}^{\prime \prime}=\left(l_{M} / l_{F}\right)^{1 / 2}$ & $(9)$ \\
\hline thermal inertia & $(k \rho c)_{s, M} /(k \rho c)_{s, F} \propto\left(l_{M} / l_{F}\right)^{3 / 2}$ & $(10)$ \\
\hline thickness & $(k / \delta)_{s, M} /(k / \delta)_{s, F} \propto\left(l_{M} / l_{F}\right)^{1 / 2}$ & \\
\hline
\end{tabular}

Assuming $\Delta H_{c, M}=\Delta H_{c, F} . l$ is the length scale. Index $M$ is related to the model scale and index $F$ to full scale. 


\section{$3 \quad$ Test set-up}

A total of 7 tests were carried out in three rooms with a scaling ratio of 1:1, 1:2 and 1:3.5 respectively. Photos of fully developed fires in the different scales are shown in Figure 1.

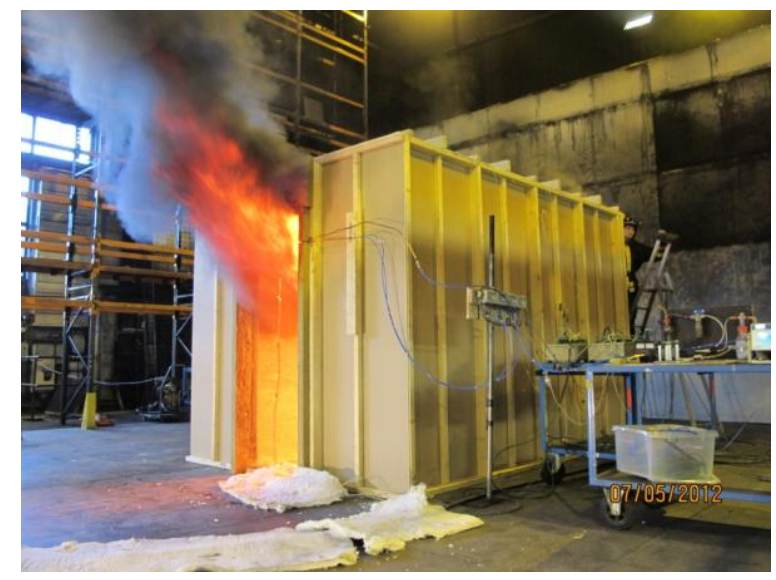

(a) $1: 1$

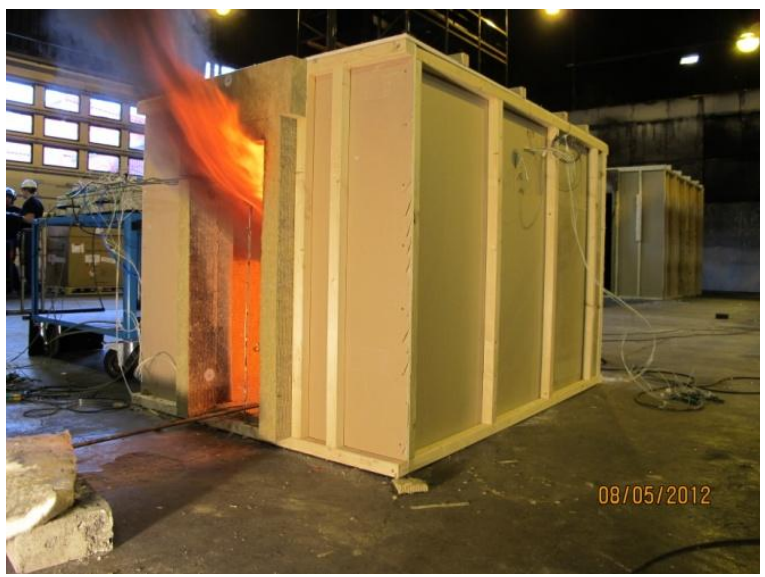

(b) $1: 2$

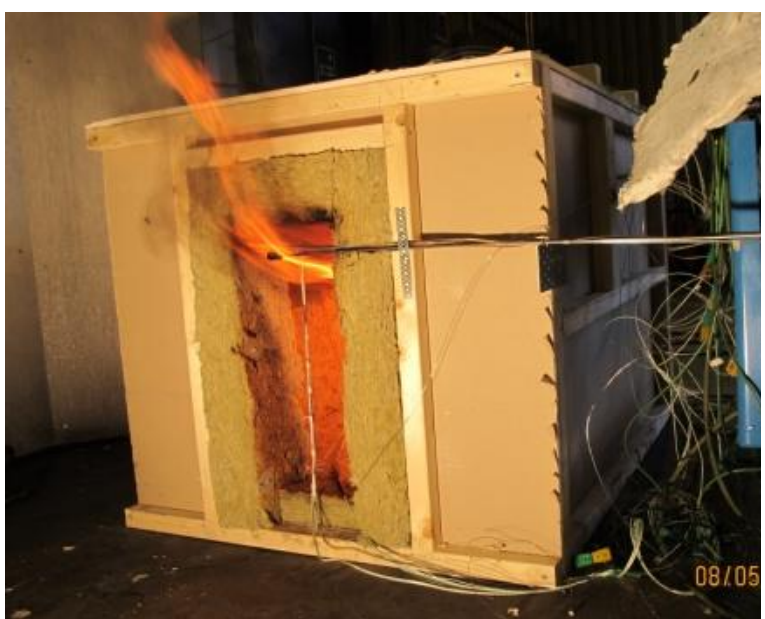

(c) $1: 3.5$

Figure 1 Photos of the room fires in different scales. The heat release rates were 1.2 MW at full scale. 


\subsection{Room geometry and structure}

All the three rooms were constructed using non-combustible $13 \mathrm{~mm}$ thick gypsum boards, mounted in wooden frames. The geometry of the room in full scale is $2.4 \mathrm{~m}(\mathrm{~W}) \times 2.4 \mathrm{~m}$ $(\mathrm{H}) \times 3.6 \mathrm{~m}(\mathrm{~L})$ and the door is $2 \mathrm{~m}$ high and $0.8 \mathrm{~m}$ wide, see Figure 2. In other words, the full scale room has the same geometry as in room corner test.

Note that the size of the room is scaled geometrically according to the scaling ratio. Therefore, in the 1:2 scale room, the geometry was $1.2 \mathrm{~m}(\mathrm{~W}) \times 1.2 \mathrm{~m}(\mathrm{H}) \times 1.8 \mathrm{~m}(\mathrm{~L})$ and the door had dimensions of $0.4 \mathrm{~m}(\mathrm{~W}) \times 1 \mathrm{~m}(\mathrm{H})$. In the 1:3.5 scale room, the geometry was $0.69 \mathrm{~m}(\mathrm{~W}) \times 0.69 \mathrm{~m}(\mathrm{H}) \times 1.03 \mathrm{~m}(\mathrm{~L})$ and the door had dimensions of $0.23 \mathrm{~m}(\mathrm{~W}) \times 0.57 \mathrm{~m}(\mathrm{H})$, see Figure 2 .

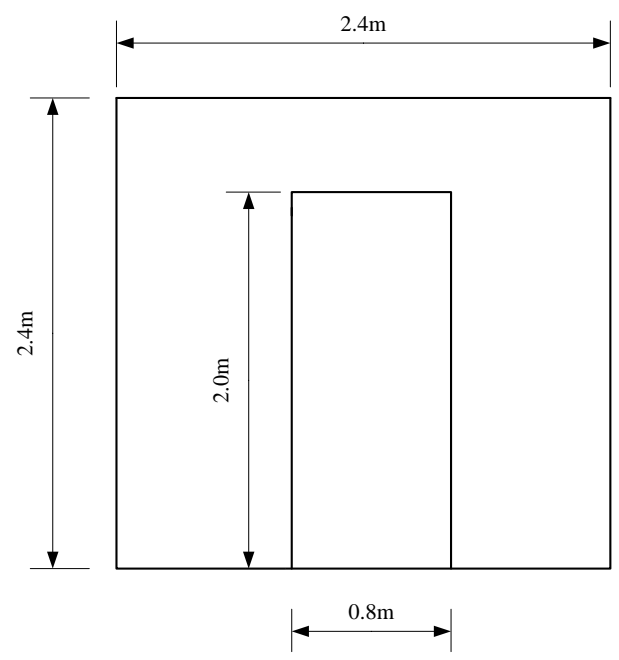

(a) $1: 1$

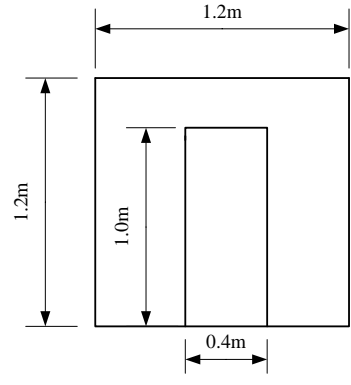

(b) $1: 2$

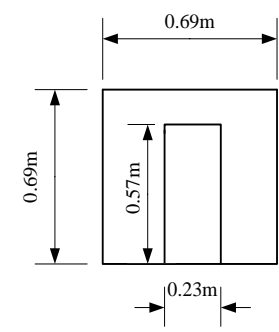

(c) $1: 3.5$

Figure 2 Front view of the rooms in different scales.

\subsection{Insulating wall materials}

The interior walls were covered with mineral wool (stone wool) in all tests. During the tests, the material was directly exposed to the hot gases. However, different types of mineral wools were used in different scales based on Eq. (4) and Eq. (5), see Table 2. In other words, more thermal resistant materials need to be used in a smaller scale. Note that the values of heat of capacity and conductivity in Table 2 are quite close to each other for these three mineral wools, however the density varies. For the scale ratio of 1 to 3.5 , it is difficult to find a mineral wool with the thickness of 109 required by the scaling laws, therefore the thickness of $120 \mathrm{~mm}$ were used instead. However, the redundancy in thickness was compensated for when measuring the temperatures inside the wall as the measurement points for the thermocouples inside the wall were situated based on the thickness of $109 \mathrm{~cm}$ as required by the scaling laws, rather than $120 \mathrm{~cm}$. Therefore it is expected that the influence of the difference in the thickness could be ignored.

Note that all the lining materials were attached to the $13 \mathrm{~mm}$ gypsum board mounted to the wooden frames. This means that the materials outside of the minerals wools were not scaled, and it may have some influence on the temperature distribution inside the wall 
after the heat penetrates the mineral wools. However, the temperature gradient at this depth is much smaller compared to in mineral wools. Further, it can be expected that the heat has not penetrated the mineral wools before $20 \mathrm{~min}$ at full scale. Therefore, it is reasonable to consider the influence of the gypsum boards as being insignificant, at least at the beginning of the tests. This effect will be investigated based on tests data.

The inner thick materials are the most important. The temperature gradients inside can be expected to be much greater than the other layers. Further, the temperatures at the backside of the insulating materials in the tests are expected to be low before $20 \mathrm{~min}$. Therefore, only the inner layer (fire resistant part) is scaled in the tests.

Further, note that the thermal conductivity could vary slightly with the temperature. However, it is expected that the conductivities for these three similar materials show similar dependences on the temperature and thus this effect is implicitly considered.

Table 2 The chosen materials based on the scaling Eqs. (9) and (10).

\begin{tabular}{ccccccccc}
\hline $\begin{array}{c}\text { Scale } \\
\text { ratio }\end{array}$ & Materials & $\begin{array}{c}\mathrm{k} \\
(\mathrm{W} / \mathrm{m} \cdot \mathrm{K})\end{array}$ & $\begin{array}{c}\rho \\
\left(\mathrm{kg} / \mathrm{m}^{3}\right)\end{array}$ & $\begin{array}{c}\mathrm{c} \\
(\mathrm{J} / \mathrm{kg} \cdot \mathrm{K})\end{array}$ & $\begin{array}{c}\text { Thermal } \\
\text { inertia } \mathrm{kpc} \\
\left(\mathrm{J}^{2} / \mathrm{m}^{4} \cdot \mathrm{K}^{2} \cdot \mathrm{s}\right)\end{array}$ & \multicolumn{2}{c}{$\begin{array}{c}\text { Thickness } \\
(\mathrm{mm})\end{array}$} \\
\hline $1: 1$ & $\begin{array}{c}\text { PAROC } \\
\text { FPS 17 }\end{array}$ & 0.038 & 170 & 750 & 4845 & 4845 & 60 & 60 \\
$1: 2$ & $\begin{array}{c}\text { PAROC ROX } \\
\text { COS 10 }\end{array}$ & 0.036 & 65 & 750 & 1713 & 1755 & 80 & 80 \\
$1: 3.5$ & PAROC UNS & 0.037 & 28 & 750 & 740 & 777 & 109 & 120 \\
\hline
\end{tabular}

\subsection{Fire sources}

Propane burners were used in the tests due to its simplicity and convenience to control the heat release rate. Note that the flame length can be expressed in such a simple form [29]:

$$
L_{f}=0.235 Q^{2 / 5}-1.02 D
$$

Note that $Q \propto l^{5 / 2}$. We obtain:

$$
D \propto l, \quad L_{f} \propto l
$$

This suggests that the geometry of the fire source should scales as the length scale. Cubic fire sources were used in the tests. The side lengths of the fire sources are $30 \mathrm{~cm}, 15 \mathrm{~cm}$ and $8.6 \mathrm{~cm}$ in different scales, respectively.

The propane burner on the floor was either placed at the center of the room or at the room corner opposite to the door, see Figure 3. The top surfaces were $30 \mathrm{~cm}, 15 \mathrm{~cm}$ and $8.6 \mathrm{~cm}$ above the floor in full scale, medium scale and small scale, respectively.

\subsection{Measurements and instrumentation}

The instrumentation of the full scale room is shown in Figure 4. In other scales, the geometrical dimensions are directly scaled by the scaling ratio. The layout of 
instrumentation in 1:2 and 1:3.5 model scales can be found in Appendix B. Here the measurements in full scale are described.

Various measurements were conducted during each test. Figure 3 shows the layout and identification of instruments in the full scale room.

One thermocouple tree was placed at the center of the room, i.e. T1 to T6. Another thermocouple tree was positioned at the centerline of the door, i.e. T7 to T11. Four thermocouples, i.e. T12 to T15 were placed $0.2 \mathrm{~m}$ below the ceiling, see Figure 3. All the thermocouples have a diameter of $0.25 \mathrm{~mm}$.

Five plate thermometers[30,31], i.e. PT1 to PT5, were either placed on the walls or on the floor of the room center. The incident heat fluxes are calculated by the following equation:

$$
\left[q_{i n c}^{\prime \prime}\right]^{i+1}=\frac{\varepsilon_{P T} \sigma\left[T_{P T}^{4}\right]^{i}+\left(h_{c, P T}+K_{\text {cond }}\right)\left(\left[T_{P T}^{4}\right]^{i}-T_{g}\right)+C_{\text {heat }, \beta=1 / 3} \frac{\left[T_{P T}\right]^{i+1}-\left[T_{P T}\right]^{i}}{t^{i+1}-t^{i}}}{\varepsilon_{P T}}
$$

where the conduction correction factor $K_{\text {cond }}=8.43 \mathrm{~W} / \mathrm{m}^{2} \cdot \mathrm{K}$, and the lumped heat capacity coefficient $C_{\text {heat }, \beta=1 / 3}=4202 \mathrm{~J} / \mathrm{m}^{2} \cdot \mathrm{K}$, the surface emissivity of Plate thermometer $\varepsilon_{P T}=0.8$.

Hot gas flow velocity through the door, i.e. BP1, was measured using a bi-directional tube [32] placed beside the centerline of the door and $0.2 \mathrm{~m}$ below the upper edge of the door in full scale. The pressure difference was measured with a pressure transducer with a measuring range of $+/-30 \mathrm{~Pa}$.

Gas concentrations through the door, including $\mathrm{CO}_{2}, \mathrm{CO}$ and $\mathrm{O}_{2}$, i.e. $\mathrm{G} 1$ to $\mathrm{G} 3$, were sampled by one probe consisting of open copper tube beside the bi-directional probe, i.e. $0.2 \mathrm{~m}$ below the upper edge of the door. 


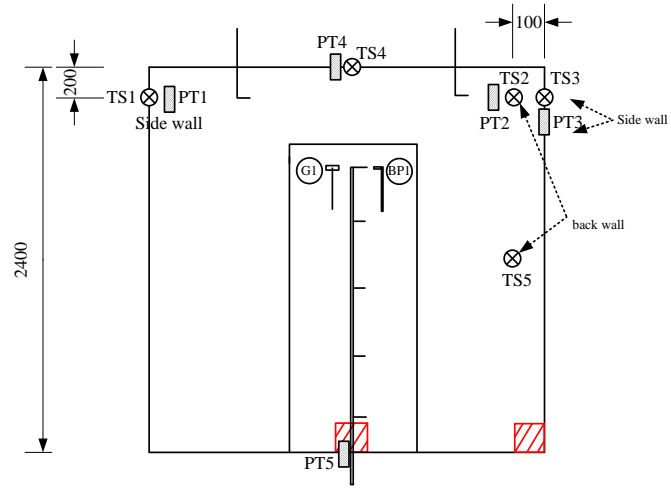

(a) front view

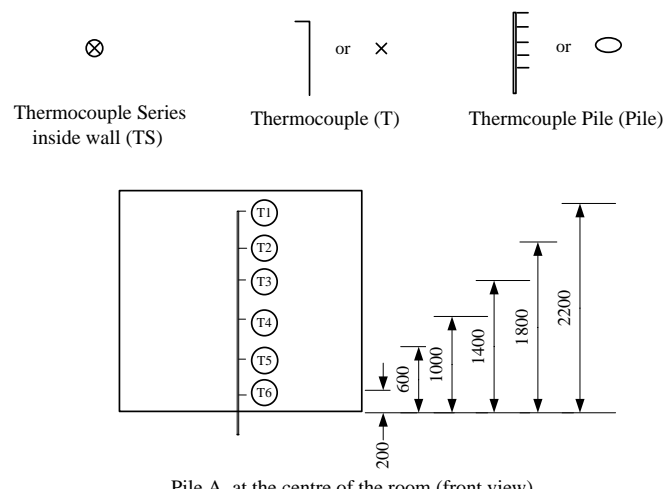

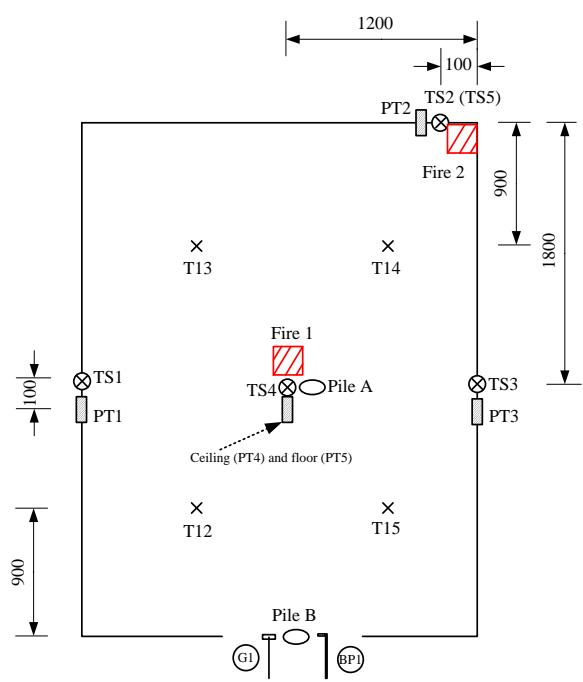

(b) top view
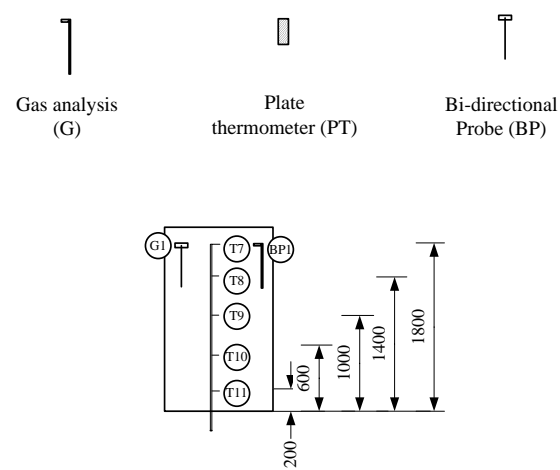

Pile B, at the door (front view)

Figure 3 Layout of instrumentation in the full scale tests (Dimensions in: $\mathrm{mm}$ ).

The thermocouple series inside the walls were placed at 5 positions in the room, i.e. TS1 to TS5, see Figure 4. At each position, a thermocouple series consisting of 5

thermocouples was placed in different depths below the interior wall surface, see Figure 5. The five depths from the interior wall surfaces are 10\%, 20\%, 40\%, $60 \%$ and $80 \%$ of the thickness (60, 80 and $109 \mathrm{~mm}$ in 3 scales respectively). Note that in different scales, the positions of corresponding thermocouples are different since the total thicknesses are different.

All the thermocouples, pressure transducers, gas analysers, flux meters, flow meter, and activation equipments were all connected to IMP 5000 KE Solotron loggers. The data were recorded by a laptop computer at a rate of approximately one scan per second. 


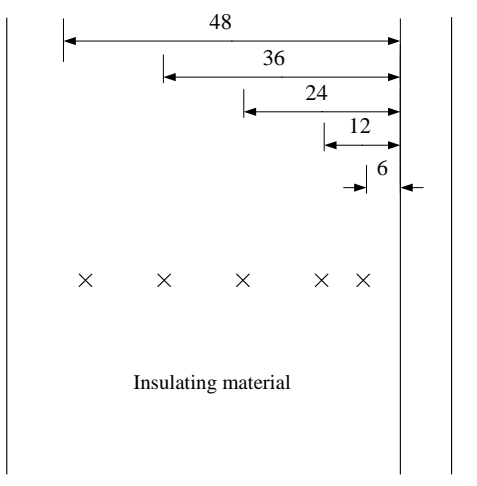

(a) 1:1

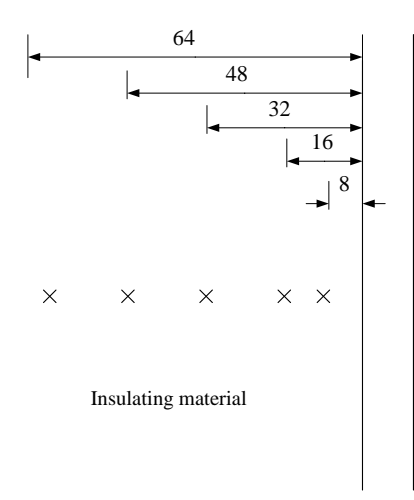

(b) $1: 2$

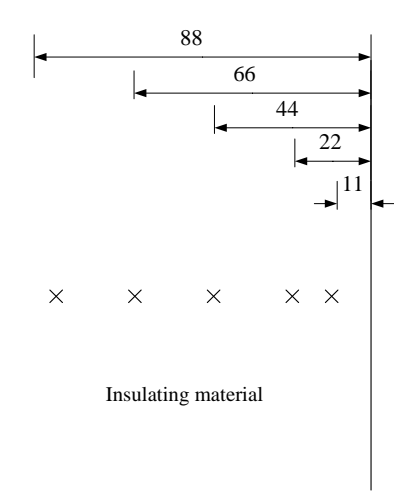

(c) $1: 3.5$

Figure 4 Locations of thermocouples inside the walls (Dimensions in: $\mathrm{mm}$ ). 


\section{Test procedure}

In total, 7 tests were carried out in different scales, see Table 2 . The main variable is the room geometry, lining materials, fire sources location and fire curves. During each test, the heat release rate was increased in a stepwise manner. In tests 1 to 3 , the heat release rate was $100 \mathrm{~kW}$ for the early $10 \mathrm{~min}$ at full scale $(0 \mathrm{~min}$ to $10 \mathrm{~min})$, and $300 \mathrm{~kW}$ for another $10 \mathrm{~min}$ at full scale (10 $\mathrm{min}$ to $20 \mathrm{~min}$ ), and then immediately turn the burner off. In tests 4 to 7 , the heat release rate was $1.2 \mathrm{MW}$ for further $7 \mathrm{~min}$ at full scale $(20 \mathrm{~min}$ to $27 \mathrm{~min}$ ) and then immediately turn the burner off. In other scales, the transition time was determined based on scaling law, i.e. Eq. (2) in Table 1. The gas burners were placed at the corner towards the door in tests 4 to 6 and placed at the center of the room in other tests. The burners were always set at floor level.

Table 3 HRR in different scales (Center and corner fires).

\begin{tabular}{ccccccccc}
\hline \multirow{2}{*}{$\begin{array}{c}\text { Test } \\
\text { nr. }\end{array}$} & Fire location & Scaling ratio & \multicolumn{2}{c}{ 1st stage } & \multicolumn{2}{c}{ 2nd stage } & \multicolumn{2}{c}{ 3rd stage } \\
\cline { 4 - 9 } & & $\begin{array}{c}\mathrm{HRR} \\
\mathrm{kW}\end{array}$ & $\begin{array}{c}\mathrm{t}_{1} \\
\text { Min }\end{array}$ & $\begin{array}{c}\mathrm{HRR} \\
\mathrm{kW}\end{array}$ & $\begin{array}{c}\mathrm{t}_{2} \\
\mathrm{~min}\end{array}$ & $\begin{array}{c}\mathrm{HRR} \\
\mathrm{kW}\end{array}$ & $\begin{array}{c}\mathrm{t}_{3} \\
\text { min }\end{array}$ \\
\hline 1 & Center & $1: 1$ & 100 & 10 & 300 & 20 & & \\
2 & Center & $1: 2$ & 18 & 7.1 & 53 & 14.1 & & \\
3 & Center & $1: 3.5$ & 4.4 & 5.3 & 13.1 & 10.7 & & \\
4 & Corner & $1: 1$ & 100 & 10 & 300 & 20 & 1200 & 27.0 \\
5 & Corner & $1: 2$ & 18 & 7.1 & 53 & 14.1 & 212 & 19.1 \\
6 & Corner & $1: 3.5$ & 4.4 & 5.3 & 13.1 & 10.7 & 52.4 & 14.4 \\
7 & Center & $1: 2$ & 18 & 7.1 & 53 & 14.1 & 212 & 19.1 \\
\hline
\end{tabular}

In each test, a cube of fibreboard was used as the ignition source. It was soaked in heptane and then placed beside the gas burner. This cube was ignited at 2 minutes from starting the logging system, and then moved after the burner was ignited. The ambient temperature was approximately $20{ }^{\circ} \mathrm{C}$ and the humidity is around $50 \%$.

Some photos of the tests are presented in Appendix C. 


\section{$5 \quad$ Summary of tests data}

A short summary of tests data are presented here for the further analysis. These data include gas temperatures measured beneath the ceiling and at the thermocouple trees, heat fluxes, gas flow and gas concentration measured at the door and temperatures inside the walls. Note that in the tables presented in this chapter, the time in model scales have been scaled up for comparison, but not for the other parameters.

All the detailed tests data can be found in Appendix D.

\subsection{Gas temperatures}

The gas temperatures measured in all the tests are given in Table 4. T1 to T6 correspond to the thermocouple tree located at the center of the room, and T7 to T11 correspond to the thermocouple tree located along the centerline of the door. T12 to T15 are the gas temperatures measured $20 \mathrm{~cm}$ beneath the ceiling.

Note that in each test, the fire heat release rate can be divided into several stages. In tests 1 to 3 , the heat release rate was $100 \mathrm{~kW}$ for the early $10 \mathrm{~min}$ in full scale $(0 \mathrm{~min}$ to 10 $\mathrm{min}$ ), and $300 \mathrm{~kW}$ for another $10 \mathrm{~min}$ in full scale (10 $\mathrm{min}$ to $20 \mathrm{~min}$ ). In tests 4 to 7 , the heat release rate was $1.2 \mathrm{MW}$ for 7 more min in full scale (20 min to $27 \mathrm{~min})$. Note that the gas temperatures increase with time at each stage, therefore, the maximum values generally correspond to the values at the last moment of the corresponding stage.

\subsection{Incident heat flux}

Incident heat fluxes measured by the plate thermometers are presented in Table 5. The maximum values generally correspond to the value at the last moment of a stage.

\subsection{Gas flow and concentration}

Gas flow velocity measured by bi-directional pressure tube and gas concentrations, including $\mathrm{CO}_{2}, \mathrm{CO}$ and $\mathrm{O}_{2}$, are presented in Table 5. The maximum values generally correspond to the value at the last moment of a stage.

\section{$5.4 \quad$ Temperatures inside the wall}

The internal wall temperatures measured using thermocouples embedded inside the insulation materials at different positions are presented in Table 6 and Table 7. Only the data corresponding to the end of each stage are listed here. Each thermocouple series (TS), includes 5 thermocouples, named 1 to 5 in the tables. Position 1 corresponds to the thermocouple closest to the interior wall surface, i.e. $10 \%$ of thermal wall thickness below the interior wall surface, and position 5 is the deepest, i.e. $80 \%$ of thermal wall thickness below the interior wall surface. 
Table 4 Test results relevant to gas temperatures.

\begin{tabular}{|c|c|c|c|c|c|c|c|c|c|c|c|c|c|c|c|c|}
\hline \multirow{2}{*}{ Test no. } & \multirow{2}{*}{$\begin{array}{l}\text { Full-scale } \\
\text { time } t \text { (min) }\end{array}$} & $\mathrm{T} 1$ & $\mathrm{~T} 2$ & T3 & $\mathrm{T} 4$ & T5 & T6 & $\mathrm{T} 7$ & $\mathrm{~T} 8$ & T9 & $\mathrm{T} 10$ & T11 & T12 & $\mathrm{T} 13$ & T14 & T15 \\
\hline & & ${ }^{\circ} \mathrm{C}$ & ${ }^{\circ} \mathrm{C}$ & ${ }^{\circ} \mathrm{C}$ & ${ }^{\circ} \mathrm{C}$ & ${ }^{\circ} \mathrm{C}$ & ${ }^{\circ} \mathrm{C}$ & ${ }^{\circ} \mathrm{C}$ & ${ }^{\circ} \mathrm{C}$ & ${ }^{\circ} \mathrm{C}$ & ${ }^{\circ} \mathrm{C}$ & ${ }^{\circ} \mathrm{C}$ & ${ }^{\circ} \mathrm{C}$ & ${ }^{\circ} \mathrm{C}$ & ${ }^{\circ} \mathrm{C}$ & ${ }^{\circ} \mathrm{C}$ \\
\hline \multirow{2}{*}{1} & 10 & 265 & 304 & 430 & 625 & 700 & 87 & 196 & 109 & 52 & 30 & 28 & 210 & 216 & 215 & 209 \\
\hline & 20 & 554 & 636 & 750 & 837 & 829 & 295 & 390 & 289 & 139 & 59 & 56 & 453 & 446 & 447 & 466 \\
\hline \multirow{2}{*}{2} & 10 & 266 & 290 & 403 & 616 & 841 & 113 & 212 & 182 & 56 & 36 & 31 & 225 & 229 & 227 & 226 \\
\hline & 20 & 504 & 512 & 637 & 695 & 811 & 213 & 386 & 343 & 143 & 76 & 54 & 466 & 421 & 435 & 462 \\
\hline \multirow{2}{*}{3} & 10 & 208 & 213 & 227 & 273 & 327 & 71 & 187 & 115 & 38 & 24 & 22 & 202 & 203 & 198 & 197 \\
\hline & 20 & 376 & 372 & 408 & 277 & 374 & 154 & 332 & 225 & 63 & 40 & 31 & 393 & 374 & 354 & 368 \\
\hline \multirow{3}{*}{4} & 10 & 289 & 231 & 113 & 94 & 76 & 62 & 237 & 73 & 50 & 30 & 27 & 267 & 274 & 297 & 269 \\
\hline & 20 & 558 & 462 & 328 & 274 & 233 & 189 & 422 & 184 & 130 & 65 & 59 & 498 & 521 & 672 & 523 \\
\hline & 27 & 981 & 1034 & 977 & 843 & 801 & 649 & 957 & 730 & 529 & 427 & 376 & 1003 & 1131 & 1181 & 1021 \\
\hline \multirow{3}{*}{5} & 10 & 312 & 236 & 110 & 97 & 80 & 68 & 245 & 84 & 57 & 34 & 24 & 290 & 307 & 335 & 300 \\
\hline & 20 & 547 & 467 & 320 & 268 & 225 & 203 & 459 & 214 & 155 & 69 & 46 & 511 & 551 & 635 & 554 \\
\hline & 27 & 923 & 925 & 885 & 827 & 686 & 701 & 873 & 708 & 518 & 310 & 179 & 914 & 1023 & 1049 & 956 \\
\hline \multirow{3}{*}{6} & 10 & 306 & 219 & 134 & 113 & 91 & 83 & 219 & 71 & 37 & 25 & 24 & 265 & 293 & 336 & 283 \\
\hline & 20 & 495 & 388 & 297 & 256 & 210 & 191 & 351 & 147 & 68 & 37 & 34 & 430 & 484 & 590 & 460 \\
\hline & 27 & 892 & 830 & 739 & 661 & 616 & 559 & 728 & 519 & 239 & 134 & 120 & 804 & 901 & 978 & 834 \\
\hline \multirow{3}{*}{7} & 10 & 274 & 370 & 498 & 594 & 807 & 102 & 214 & 173 & 54 & 37 & 33 & 229 & 227 & 225 & 226 \\
\hline & 20 & 507 & 593 & 642 & 729 & 825 & 267 & 383 & 340 & 133 & 79 & 71 & 441 & 413 & 419 & 458 \\
\hline & 27 & 896 & 888 & 866 & 784 & 902 & 623 & 983 & 861 & 598 & 463 & 397 & 962 & 857 & 857 & 933 \\
\hline
\end{tabular}


Table 5 Test results relevant to heat flux, gas flow velocity and gas concentration.

\begin{tabular}{|c|c|c|c|c|c|c|c|c|c|c|}
\hline \multirow{2}{*}{$\begin{array}{l}\text { Test } \\
\text { no. }\end{array}$} & \multirow{2}{*}{$\begin{array}{c}\text { Full-scale } \\
\text { time } t \\
\text { (min) }\end{array}$} & PT1 & PT2 & PT3 & PT4 & PT5 & $u$ & $\mathrm{CO} 2$ & $\mathrm{CO}$ & $\mathrm{O} 2$ \\
\hline & & $\mathrm{kW} / \mathrm{m}^{2}$ & $\mathrm{~kW} / \mathrm{m}^{2}$ & $\mathrm{~kW} / \mathrm{m}^{2}$ & $\mathrm{~kW} / \mathrm{m}^{2}$ & $\mathrm{~kW} / \mathrm{m}^{2}$ & $\mathrm{~m} / \mathrm{s}$ & $\%$ & $\%$ & $\%$ \\
\hline \multirow{2}{*}{1} & 10 & 3.7 & 2.3 & 3.4 & 4.7 & 3.7 & 3.1 & 1.05 & 0.0025 & 19.4 \\
\hline & 20 & 16.2 & 12.9 & 17.6 & 18.3 & 11.1 & 5.1 & 2.45 & 0.022 & 17.1 \\
\hline \multirow{2}{*}{2} & 10 & 4.2 & 2.0 & 3.8 & 5.7 & 3.7 & 1.6 & 1.13 & 0.0043 & 19.1 \\
\hline & 20 & 13.0 & 9.8 & 14.4 & 15.8 & 11.0 & 2.5 & 2.20 & 0.049 & 17.2 \\
\hline \multirow{2}{*}{3} & 10 & 3.4 & 1.8 & 2.9 & 4.0 & 2.9 & 0.9 & - & 0.0033 & 19.5 \\
\hline & 20 & 9.2 & 5.6 & 6.9 & 9.7 & 7.0 & 1.4 & - & 0.016 & 17.8 \\
\hline \multirow{3}{*}{4} & 10 & 4.7 & 14.2 & 5.2 & 3.6 & 2.7 & 2.3 & 1.04 & 0.0077 & 19.2 \\
\hline & 20 & 20.2 & 87.6 & 32.0 & 18.5 & 11.4 & 4.6 & 2.12 & 0.037 & 17.6 \\
\hline & 27 & 197.2 & 291.8 & 207.6 & 198.0 & 131.5 & 8.5 & 7.43 & 0.14 & 8.5 \\
\hline \multirow{3}{*}{5} & 10 & 3.4 & 11.7 & 3.8 & 3.1 & 2.6 & 1.0 & 1.05 & 0.014 & 18.8 \\
\hline & 20 & 17.1 & 67.5 & 23.0 & 16.6 & 12.4 & 2.0 & 2.37 & 0.14 & 16.6 \\
\hline & 27 & 120.7 & 200.1 & 139.4 & 123.3 & 100.1 & 4.1 & 9.52 & 0.53 & 5.5 \\
\hline \multirow{3}{*}{6} & 10 & 3.1 & 6.3 & 3.2 & 1.9 & 2.9 & 0.5 & 0.67 & 0.011 & 19.4 \\
\hline & 20 & 10.4 & 32.2 & 13.1 & 8.7 & 9.9 & 1.1 & 1.42 & 0.044 & 18.0 \\
\hline & 27 & 71.6 & 121.5 & 81.4 & 71.6 & 61.2 & 2.6 & 8.91 & 0.86 & 5.5 \\
\hline \multirow{3}{*}{7} & 10 & 4.3 & 2.1 & 4.6 & 6.0 & 3.4 & 1.7 & 1.31 & 0.0036 & 19.0 \\
\hline & 20 & 11.9 & 9.3 & 12.9 & 15.0 & 11.5 & 3.0 & 2.49 & 0.017 & 17.1 \\
\hline & 27 & 107.8 & 84.9 & 106.2 & 110.6 & 89.3 & 5.1 & - & 0.39 & 3.1 \\
\hline
\end{tabular}

"_"measurement error or out of range. 
Table 6 Test results relevant to internal wall temperatures (TS1 to TS3).

\begin{tabular}{|c|c|c|c|c|c|c|c|c|c|c|c|c|c|c|c|c|}
\hline \multirow[t]{2}{*}{$\begin{array}{l}\text { Test } \\
\text { no. }\end{array}$} & \multirow{2}{*}{$\begin{array}{c}\begin{array}{c}\text { Full scale } \\
\text { time } t\end{array} \\
\text { min }\end{array}$} & \multicolumn{5}{|c|}{ TS1 (Positions 1to 5) } & \multicolumn{5}{|c|}{$\mathrm{TS} 2$} & \multicolumn{5}{|c|}{ TS3 } \\
\hline & & 1 & 2 & 3 & 4 & 5 & 1 & 2 & 3 & 4 & 5 & 1 & 2 & 3 & 4 & 5 \\
\hline \multirow{2}{*}{1} & 10 & 81 & 66 & 29 & 22 & 20 & 97 & 64 & 34 & 27 & 21 & 102 & 58 & 38 & 25 & 21 \\
\hline & 20 & 235 & 200 & 83 & 41 & 26 & 271 & 191 & 101 & 71 & 30 & 294 & 185 & 119 & 56 & 30 \\
\hline \multirow{2}{*}{2} & 10 & 79 & 55 & 33 & 24 & 22 & 95 & 69 & 36 & 26 & 22 & 116 & 89 & 43 & 26 & 23 \\
\hline & 20 & 228 & 168 & 91 & 40 & 27 & 248 & 195 & 100 & 51 & 30 & 311 & 263 & 139 & 61 & 33 \\
\hline \multirow{2}{*}{3} & 10 & 88 & 60 & 28 & 19 & 17 & 42 & 63 & 27 & 19 & 18 & 101 & 63 & 34 & 26 & 21 \\
\hline & 20 & 242 & 187 & 94 & 37 & 23 & 122 & 169 & 81 & 40 & 29 & 244 & 180 & 106 & 77 & 55 \\
\hline \multirow{3}{*}{4} & 10 & 95 & 76 & 30 & 21 & 19 & 302 & 193 & 83 & 53 & 23 & 123 & 67 & 41 & 24 & 20 \\
\hline & 20 & 272 & 234 & 99 & 46 & 27 & 778 & 669 & 489 & 393 & 119 & 408 & 287 & 195 & 84 & 38 \\
\hline & 27 & 883 & 813 & 460 & 279 & 182 & - & - & - & - & - & - & - & - & - & - \\
\hline \multirow{3}{*}{5} & 10 & 100 & 67 & 26 & 21 & 19 & 472 & 410 & 214 & 56 & 31 & 76 & 47 & 25 & 20 & 20 \\
\hline & 20 & 343 & 283 & 96 & 53 & 30 & 688 & 641 & 510 & 324 & 112 & 341 & 237 & 64 & 38 & 28 \\
\hline & 27 & 803 & 698 & 375 & 225 & 58 & - & - & - & - & - & - & - & - & - & - \\
\hline \multirow{3}{*}{6} & 10 & 123 & 84 & 37 & 23 & 21 & - & 381 & 194 & 70 & 38 & 152 & 95 & 50 & 42 & 30 \\
\hline & 20 & 298 & 233 & 121 & 48 & 28 & - & 588 & 474 & 336 & 234 & 378 & 302 & 209 & 182 & 135 \\
\hline & 27 & 706 & 630 & 448 & 192 & 61 & - & 754 & 633 & 508 & 391 & 760 & 681 & 571 & 532 & 450 \\
\hline \multirow{3}{*}{7} & 10 & 79 & 54 & 31 & 22 & 21 & 92 & 68 & 34 & 24 & 21 & 115 & 81 & 42 & 25 & 22 \\
\hline & 20 & 212 & 158 & 86 & 37 & 25 & 237 & 187 & 96 & 48 & 28 & 289 & 224 & 128 & 57 & 31 \\
\hline & 27 & 675 & 556 & 356 & 121 & 40 & 683 & 597 & 378 & 174 & 60 & 781 & 673 & 500 & 238 & 80 \\
\hline
\end{tabular}

“_" measurement failure. 
Table 7 Test results relevant to internal wall temperatures (TS4 to TS5).

\begin{tabular}{|c|c|c|c|c|c|c|c|c|c|c|c|}
\hline \multirow[t]{2}{*}{$\begin{array}{c}\text { Test } \\
\text { no. }\end{array}$} & \multirow{2}{*}{$\begin{array}{c}\text { Full scale } \\
\text { time } t \\
\text { (min) }\end{array}$} & \multicolumn{5}{|c|}{ TS4 } & \multicolumn{5}{|c|}{ TS5 } \\
\hline & & 1 & 2 & 3 & 4 & 5 & 1 & 2 & 3 & 4 & 5 \\
\hline \multirow{2}{*}{1} & 10 & 121 & 69 & 33 & 24 & 21 & 54 & 45 & 26 & 20 & 19 \\
\hline & 20 & 334 & 214 & 99 & 46 & 28 & 163 & 136 & 63 & 30 & 22 \\
\hline \multirow{2}{*}{2} & 10 & 117 & 94 & 49 & 30 & - & 66 & 45 & 28 & 22 & 21 \\
\hline & 20 & 314 & 270 & 154 & 72 & - & 177 & 123 & 63 & 32 & 24 \\
\hline \multirow{2}{*}{3} & 10 & 76 & 50 & 29 & 19 & 18 & 47 & 32 & 20 & 18 & 17 \\
\hline & 20 & 199 & 147 & 81 & 34 & 25 & 128 & 89 & 43 & 26 & 20 \\
\hline \multirow{3}{*}{4} & 10 & 129 & 68 & 32 & 21 & 19 & 443 & 367 & 158 & 40 & 25 \\
\hline & 20 & 342 & 216 & 100 & 43 & 27 & 733 & 672 & 466 & 215 & 65 \\
\hline & 27 & 977 & 735 & 420 & 202 & 175 & 1040 & 963 & 771 & 469 & 196 \\
\hline \multirow{3}{*}{5} & 10 & 104 & 71 & 31 & 22 & 20 & 507 & 427 & 222 & 58 & 28 \\
\hline & 20 & 326 & 254 & 119 & 51 & 32 & 638 & 584 & 447 & 270 & 80 \\
\hline & 27 & 765 & 541 & 257 & 74 & 45 & 859 & 787 & 634 & 463 & 203 \\
\hline \multirow{3}{*}{6} & 10 & 90 & 55 & 32 & 22 & 21 & 423 & 314 & 109 & 36 & 22 \\
\hline & 20 & 246 & 172 & 94 & 38 & 28 & 528 & 493 & 343 & 192 & 72 \\
\hline & 27 & 644 & 534 & 360 & 127 & 59 & 720 & 685 & 581 & 452 & 268 \\
\hline \multirow{3}{*}{7} & 10 & 121 & 91 & 53 & 28 & 24 & 62 & 42 & 26 & 21 & 20 \\
\hline & 20 & 293 & 239 & 155 & 61 & 34 & 172 & 119 & 60 & 30 & 22 \\
\hline & 27 & 697 & 531 & 356 & 105 & 44 & 733 & 653 & 486 & 171 & 42 \\
\hline
\end{tabular}

“_” measurement failure. 


\section{Discussion of results}

Firstly, the measured gas temperatures, gas flows, gas concentration, and incident heat fluxes are discussed. Then the internal wall temperatures and energy balance are analyzed. Further, the calculation of upper layer gas temperatures are investigated. Note that for comparison, the time in model scales has been scaled up in most of the figures.

\subsection{Scaling of gas temperature 6.1.1 Ceiling gas temperature}

Figure 5 shows the measured ceiling gas temperature as a function of the full-scale time for center fires. In other words, the time in medium scale and small scale has been scaled up for easy comparison.

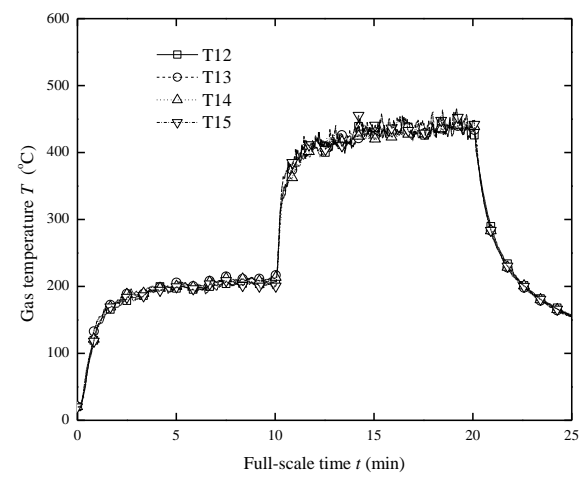

(a) Full scale

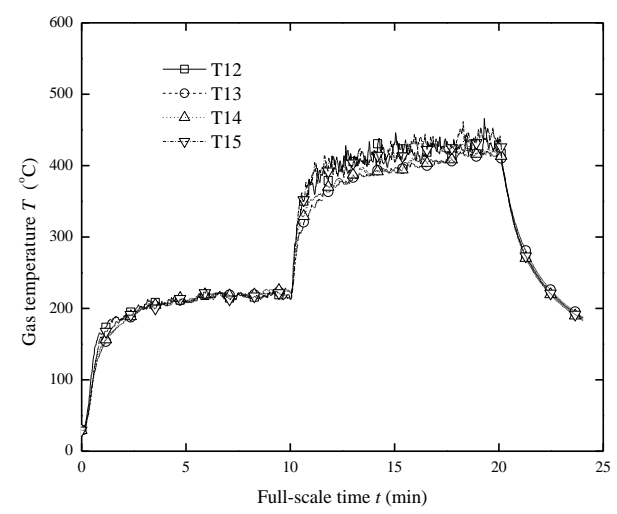

(b) Medium scale

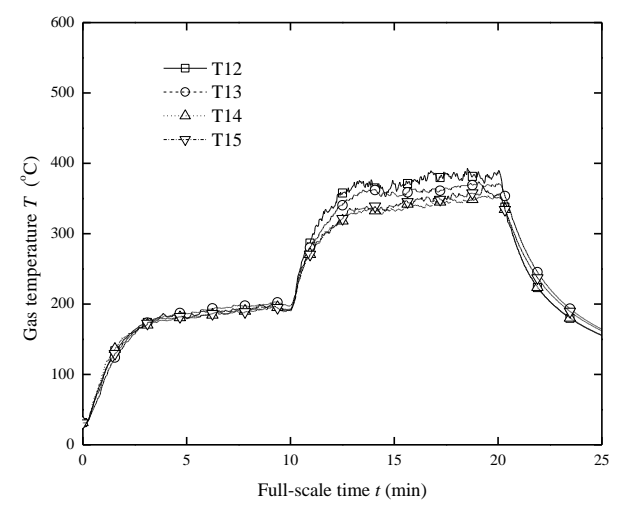

(c) Small scale

Figure 5 Ceiling gas temperatures for the center fires.

Clearly, it is shown in Figure 5 that the ceiling gas temperatures correlate well in different scales of center fires, especially in medium scale tests where a perfect match can be found. 
Note that in small scales, the ceiling gas temperatures are slightly lower, especially at the 2 nd stage when the gas temperatures are around $400{ }^{\circ} \mathrm{C}$. Further, the ceiling gas temperatures measured by the four ceiling thermocouples in full scale are closely of the same values, however, in model scales, small differences can be found for different thermocouples. In general, the ceiling gas temperatures are scaled very well in this series of tests.

Figure 6 shows the measured ceiling gas temperatures for corner fires. Note that for comparison, the time in model scales has also been scaled up.

Clearly, it is shown in Figure 6 that the ceiling gas temperatures in different scales correlate very well before $20 \mathrm{~min}$, and after $20 \mathrm{~min}$, the ceiling gas temperatures are highest in full scale, and slightly lower in medium scale and lowest in small scale. The maximum ceiling gas temperatures are $1181{ }^{\circ} \mathrm{C}$ in full scale, $1049{ }^{\circ} \mathrm{C}$ in medium scale and $978{ }^{\circ} \mathrm{C}$ in small scale. Note that these temperatures correspond to flame temperatures in the continuous combustion region and thus they partly indicate the combustion intensity. Another reason for the variations could be that the sooty smoke blocks the heat loss in larger scales.

Note that before $10 \mathrm{~min}$, all the temperatures measured at T12 to T15 are almost the same, however, after $10 \mathrm{~min}$, the temperature at T14 is much higher compared to other positions and the temperatures measured by the other thermocouples are very similar. This is due to that after $10 \mathrm{~min}$ the continuous flame region reached the ceiling and T14 directly measured the flame temperature. Also, note that the highest temperatures were registered at T14, then T13, T15 and T12. This is mainly due to that in the fully developed fires, more heat was lost at T15 and T12 which were close to the openings, and T12 is the farthest thermocouple from the fire source. This indicates that after the flame impinges the ceiling, the ceiling gas temperatures are not uniform, but depend on its location relative to the fire source and the opening.

In summary, for center fires, the upper layer gas temperatures correlate well in different scales, especially in medium scale tests where a perfect match can be found. In small scales, the ceiling gas temperatures are slightly lower, especially at the 2 nd stage. For corner fires, the ceiling gas temperatures are scaled very well before $20 \mathrm{~min}$ but are slightly underestimated in model scales after $20 \mathrm{~min}$ at a heat release rate of $1.2 \mathrm{MW}$ at full scale.

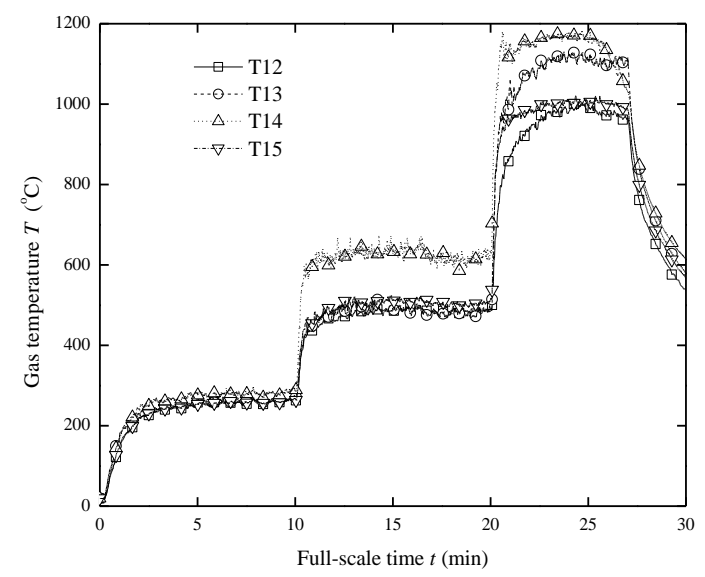

(a) Full scale 


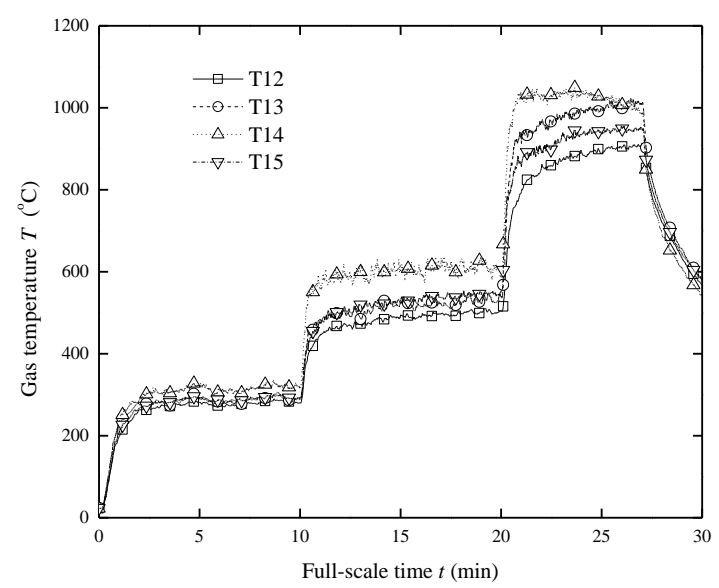

(b) Medium scale

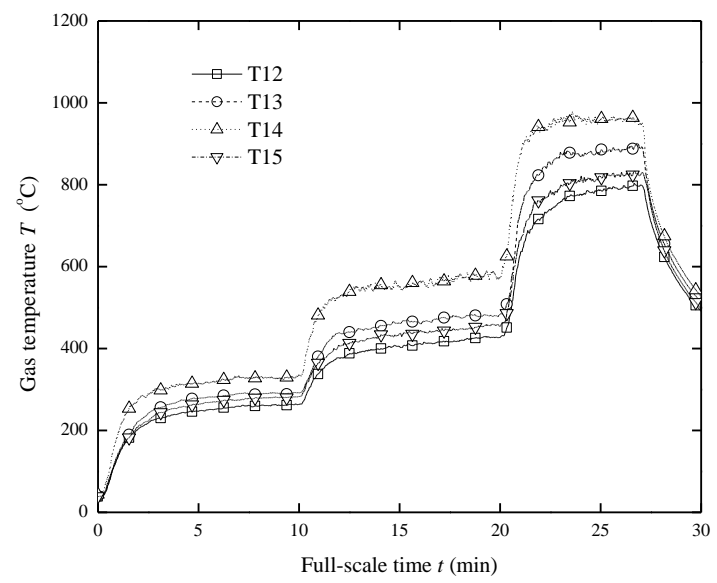

(c) Small scale

Figure $6 \quad$ Ceiling gas temperature in small scale (corner fire).

\subsubsection{Thermocouple tree at the room center}

Here the gas temperatures measured by the thermocouple trees placed in the centers of the rooms in different scales, i.e. T1 to T6, are compared.

Figure 7 shows the vertical temperature distribution in the center of the room for center fires. Note that for easy comparison, the time in model scales has been scaled up. Since the fire was placed in the center and on the floor, it was strongly affected by the air flows through the doors induced by the hot smoke flows. The scatter has been eased by averaging the measured values, i.e. $10 \mathrm{~s}$ average values in full scale, $7 \mathrm{~s}$ average in medium scale and $5 \mathrm{~s}$ in small scale according to the scaling of time. It can be seen that the measured temperatures at T4 and T5 are always instable and differ significantly in different scales. This is mainly due to the strong influence of the air flows through the openings. However, it can also be seen that there is good agreement in different scales for the measured temperatures at the other positions. 


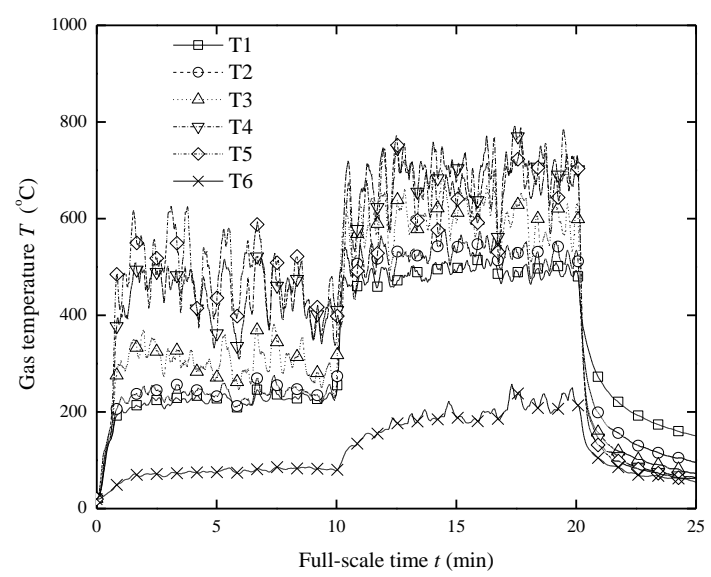

(a) Full scale

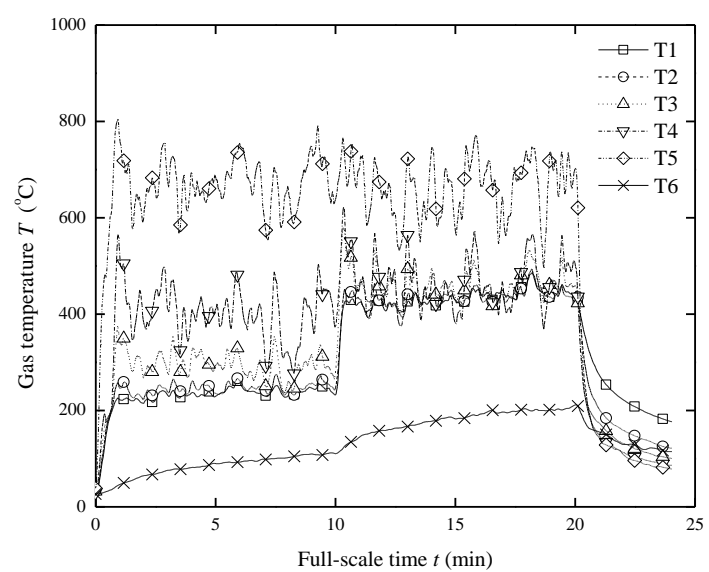

(b) Medium scale

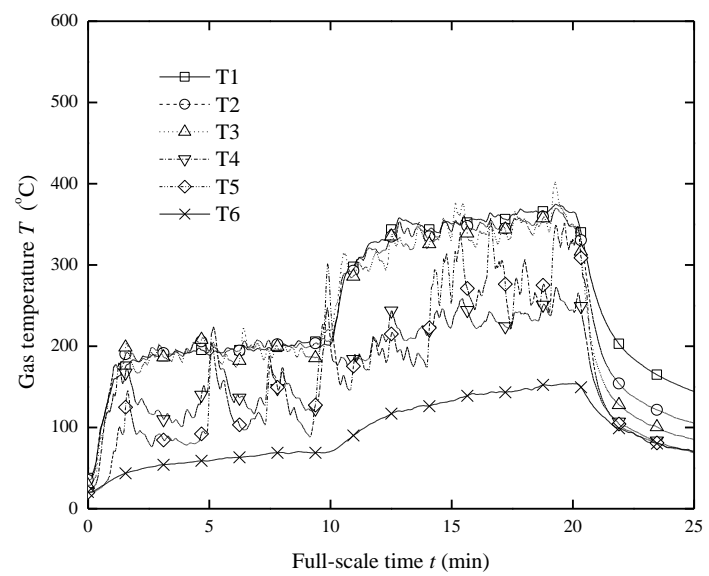

(c) Small scale

Figure $7 \quad$ Temperature distribution in center of the room for center fires.

Figure 8 shows the vertical temperature distribution in the center of the room for corner fires. Note that for comparison, the time in model scales has been scaled up. Clearly, the measured temperatures in different scales show high similarity. Before $10 \mathrm{~min}$, there was a strong stratification and the smoke layer height lay between T2 and T3. After $10 \mathrm{~min}$, the smoke layer slightly descended. After 20 min the floor temperature was over $600{ }^{\circ} \mathrm{C}$ and there was no clear stratification. 


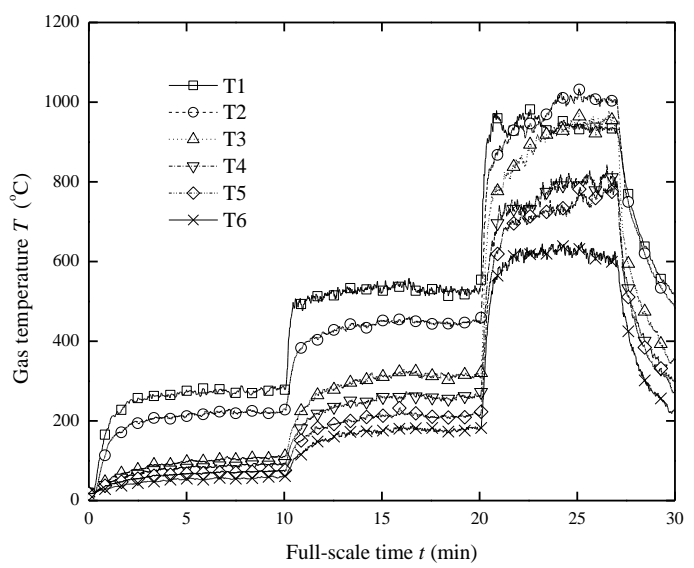

(a) Full scale

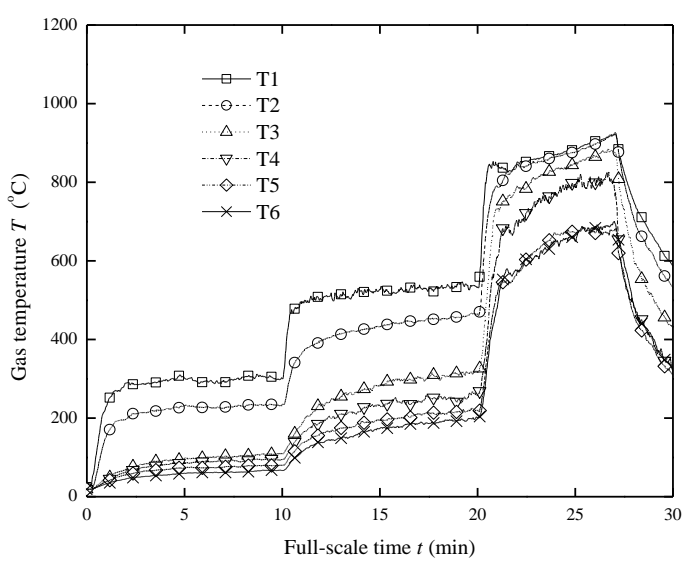

(b) Medium scale

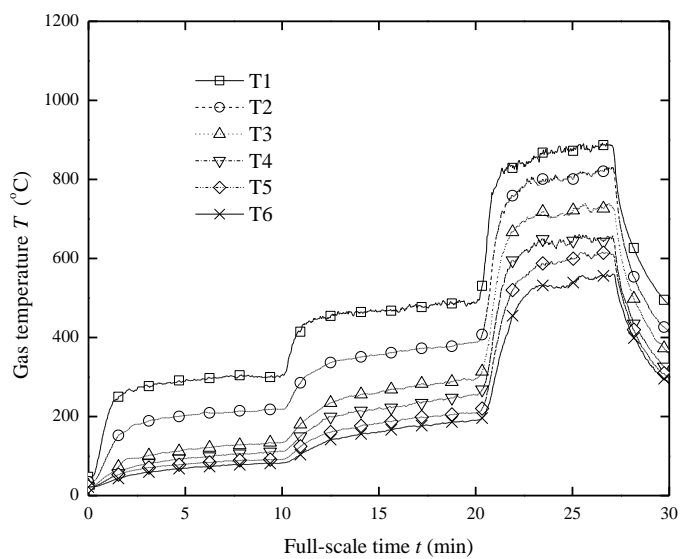

(c) Small scale

Figure 8 Temperature distribution in center of the room for corner fires.

In summary, the vertical temperature distribution at the center of the room is scaled well for both corner fires and center fires, except some data points in center fires scattered due to the strong influence of the air flows through the openings on the flame.

\subsubsection{Thermocouple tree at door}

Figure 9 shows the vertical temperature distribution at the doors for center fires in different scales. For comparison, the time in model scales has been scaled up. The smoke flow height can be estimated from the temperature distribution. It can be seen that the temperatures are scaled well in medium scale except at T8. This could be due to that the 
temperature gradient is very large and thus the gas temperature is sensitive to even slight deviation of the position of the thermocouple.

It can also be seen that in small scale the smoke layer height are slightly higher. This could be partly due to the larger roughness of the door and all the surfaces. Also, note that the temperature is lower in model scales. One reason could be that more heat is lost through the opening and the radiation is overestimated at this location in model scales. Note that for the sooty smoke inside the carriage and far away from the openings, the openings could be considered as invisible, and thus the gas temperatures can be scaled well. However, for the gases close to the opening, the radiation heat loss is significant

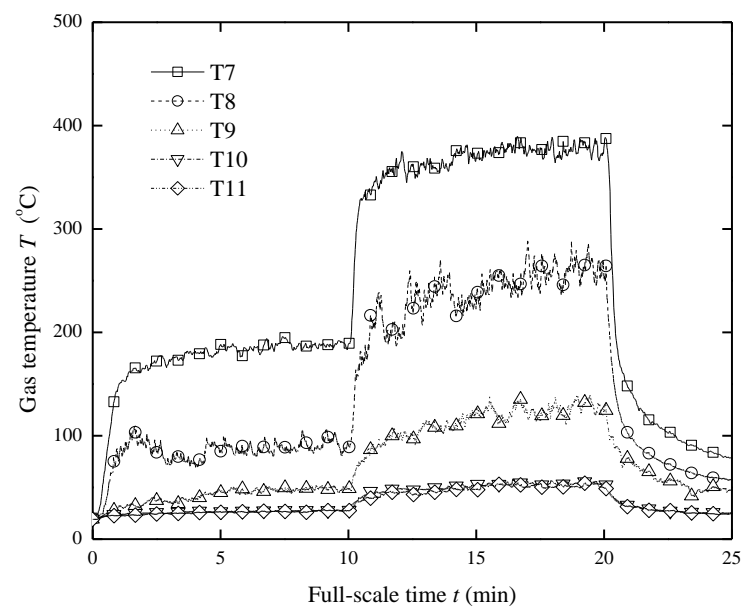

(a) Full scale

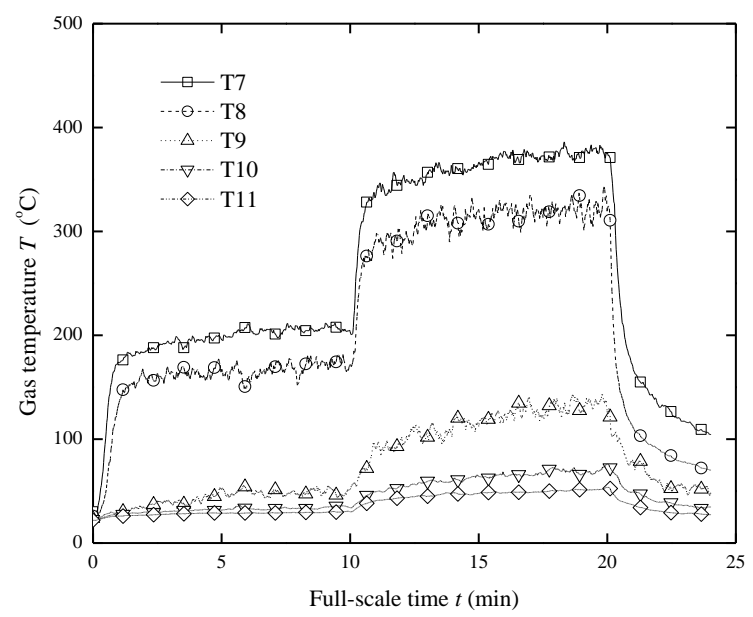

(b) Medium scale 


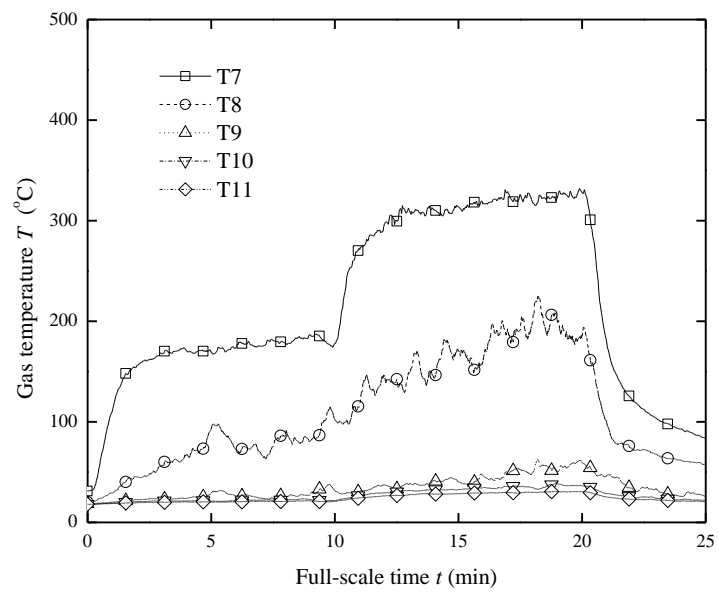

(c) Small scale

Figure 9 Temperature distribution at the doors for center fires.

Figure 10 shows the vertical temperature distribution at the doors for corner fires in different scales. For comparison, the time in model scales has been scaled up. The profiles of the temperatures have high similarities, especially between medium scale and full scale before $20 \mathrm{~min}$. However, after $20 \mathrm{~min}$, the gas temperatures at the top and at the bottom in model scales are lower than in full scale, especially in small scale.

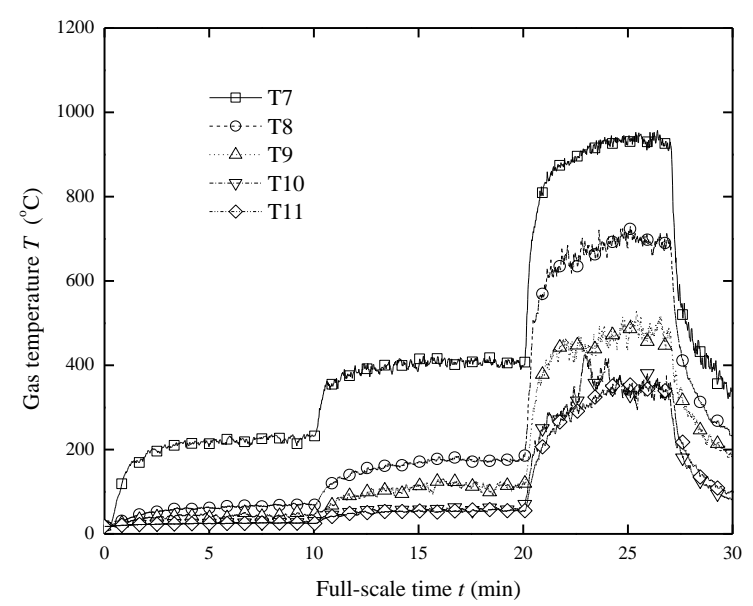

(a) Full scale

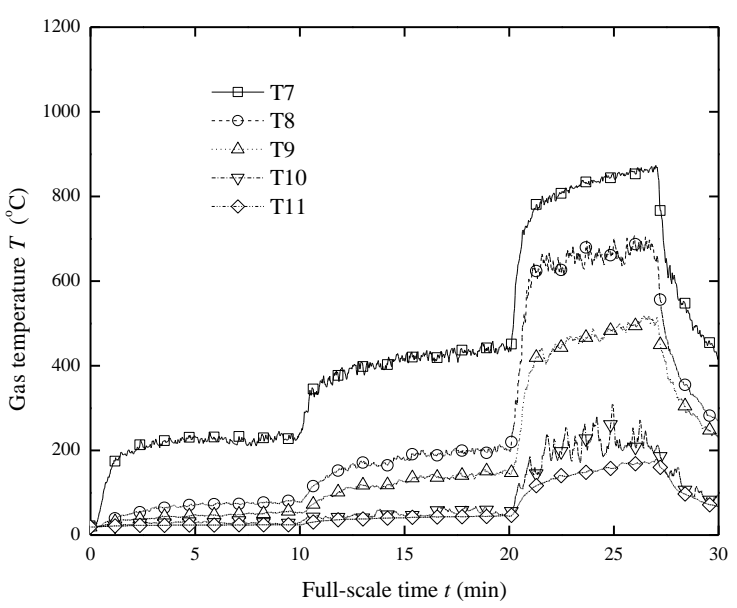

(b) Medium scale 


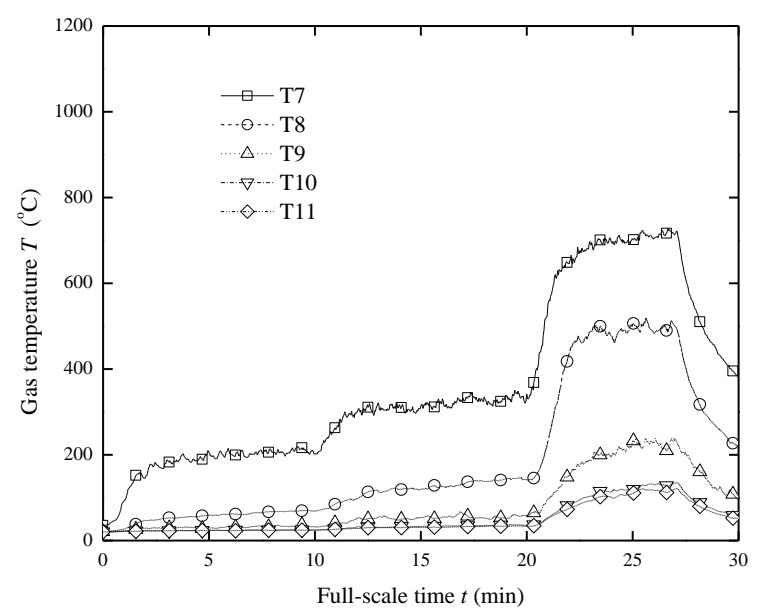

(c) Small scale

Figure 10 Temperature distribution at the doors for corner fires.

In short, the vertical temperature distribution at the door is relatively scaled well before $20 \mathrm{~min}$, however, after $20 \mathrm{~min}$, the gas temperatures at the top and at the bottom in model scales are lower in model scales compared to in full scale. This should be mainly explained by the relatively higher heat loss through the openings in model scales.

\subsection{Scaling of gas flow and concentration}

\subsubsection{Gas flow}

Figure 11 shows the gas velocities through the doors in different scales of center fires. Note that for easy comparison, the time in model scales has been scaled up. Clearly, it shows very good correlation in different scales. Note that after $10 \mathrm{~min}$, the gas velocity is slightly lower in small scales. This could be explained by the finding from the temperature distribution that the smoke layer height is slightly higher in small scale. An interesting phenomenon is the high similarity in the gas flow after 20 min when there was no fire source. Therefore, this process was a cooling process by natural ventilation, contrary to the heating process at the two early stages.

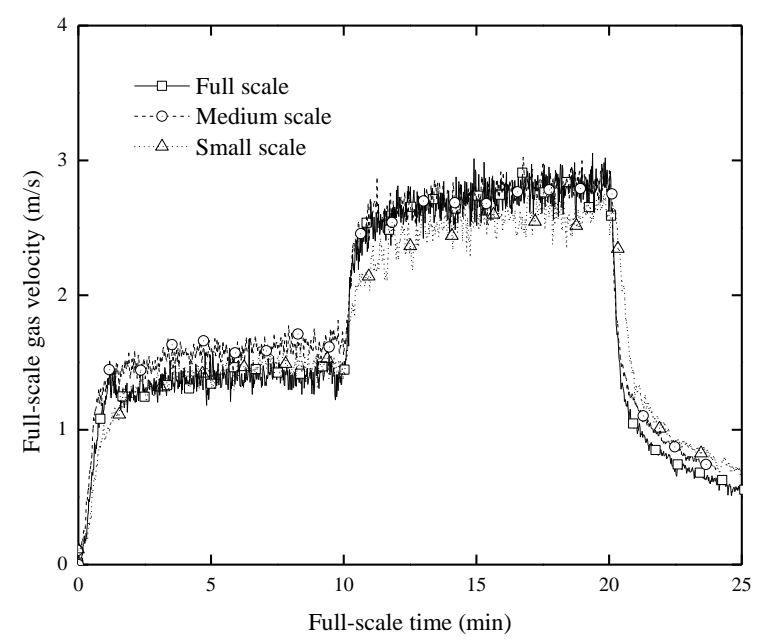

Figure $11 \quad$ Full-scale gas velocity through the doors for center fires (data scaled up). 
Figure 12 shows the gas velocities through the doors in different scales of corner fires. Also, there is good correlation between the scales. In small scale, the gas velocity is also slightly lower during $10 \mathrm{~min}$ to $20 \mathrm{~min}$, and the same reason can be used to explain this.

In summary, the gas velocities are scaled very well for both center fires and corner fires.

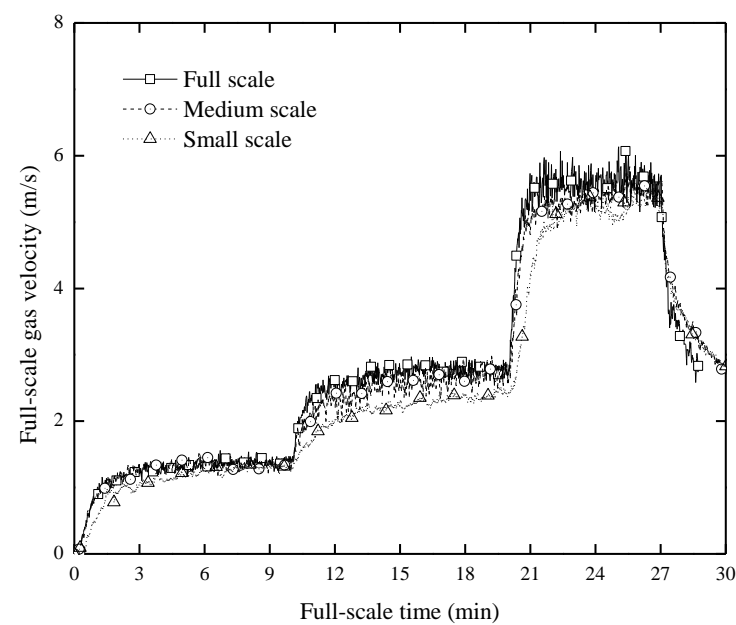

Figure 12 Full-scale gas velocity through the doors for corner fires (data scaled up).

\subsubsection{Gas concentration}

Figure 13 shows the measured oxygen concentration at the doors for center fires. Note that for easy comparison, the time in model scales has been scaled up. Clearly it shows that the medium scale perfectly matches the full scale. The oxygen concentration in small scale is slightly higher and there appears to be a delay. The reasons could be that the response time to correct the fuel flow has been scaled up, and the smoke layer height could be slightly higher in small scale.

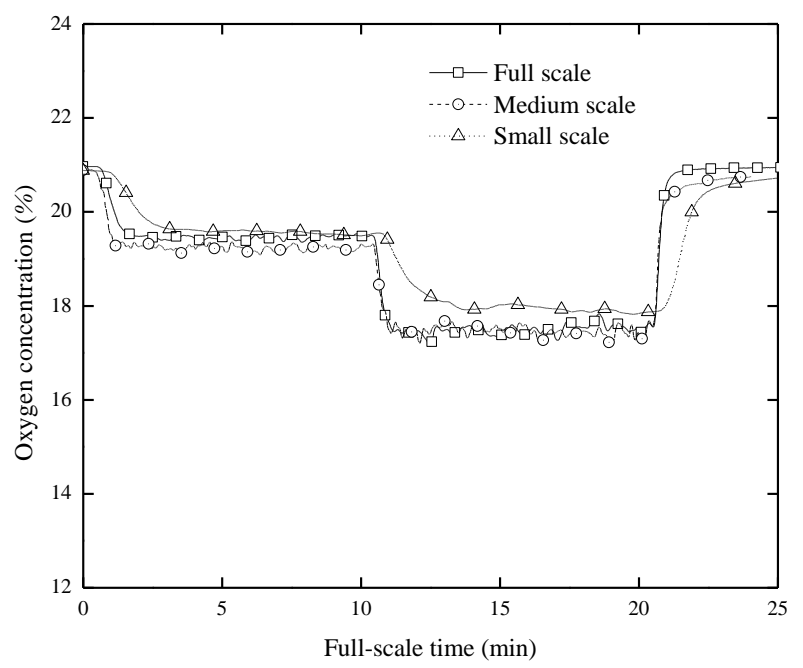

Figure $13 \mathrm{O}_{2}$ concentration at the doors for center fires.

Figure 14 shows the measured oxygen concentration at the doors for corner fires. Note that for easy comparison, the time in model scales has been scaled up. Before $20 \mathrm{~min}$, the same phenomenon can be observed as for center fires. After $20 \mathrm{~min}$, the oxygen concentration in model scales are lower. There could be three reasons for the underestimation for large fires. Firstly, the smoke layer at the door in model scales could be slightly thinner according to the vertical temperature distributions at the doors, 
especially in small scale. Secondly, the binders in the mineral wools may release some heat. Thirdly, slightly more fuel could be burnt in the room in model scales compared to the full scale due to the scaled-up experimental error.

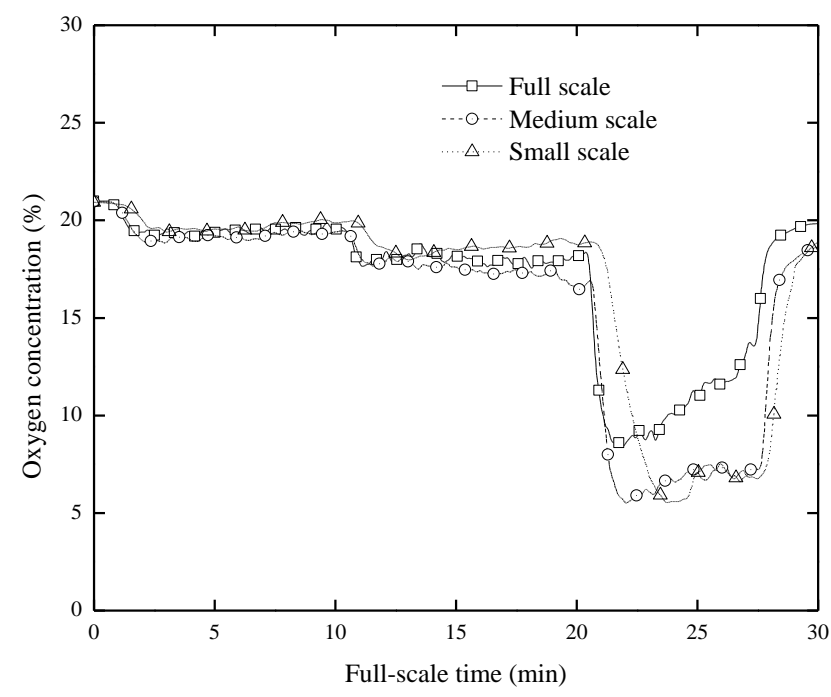

Figure $14 \mathrm{O}_{2}$ concentration at the doors for corner fires.

Figure 15 shows the measured $\mathrm{CO}_{2}$ concentration at the doors for center fires. Note that for easy comparison, the time in model scales has been scaled up. Clearly it shows that the medium scale perfectly matches the full scale. The data of small scale are not presented due to the failure of the measurement.

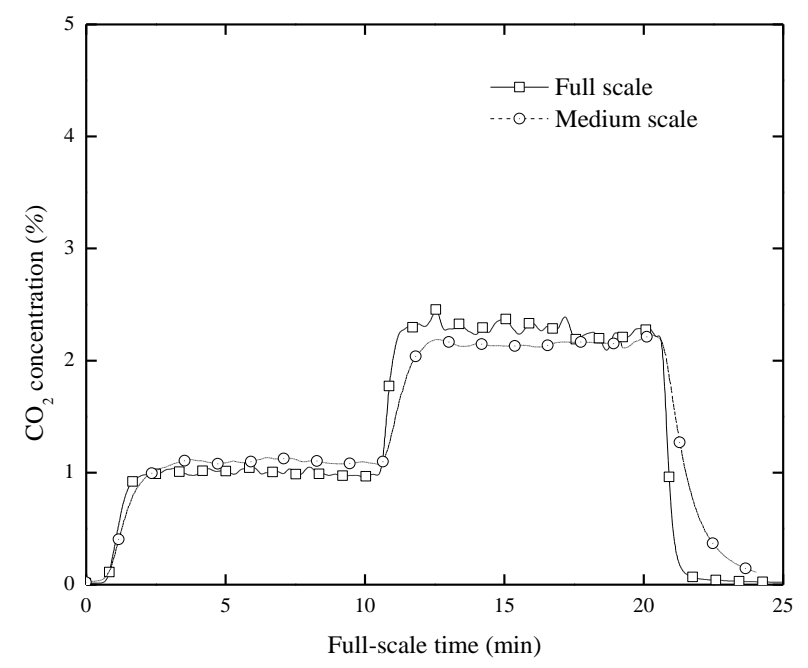

Figure $15 \mathrm{CO}_{2}$ concentration at door for center fires.

Figure 16 shows the measured $\mathrm{CO}_{2}$ concentration at the doors for corner fires. The same phenomenon as observed in $\mathrm{O} 2$ concentration can be found here. The same reasons can explain this. Note that the $\mathrm{CO} 2$ concentration increases very slowly with time at the early stage. The reason is that the smoke layers for the corner fires are slightly thinner compared to the center fires and the smoke layer height decreases gradually as the ceiling gas temperature increases with time due to the decreasing heat loss. 


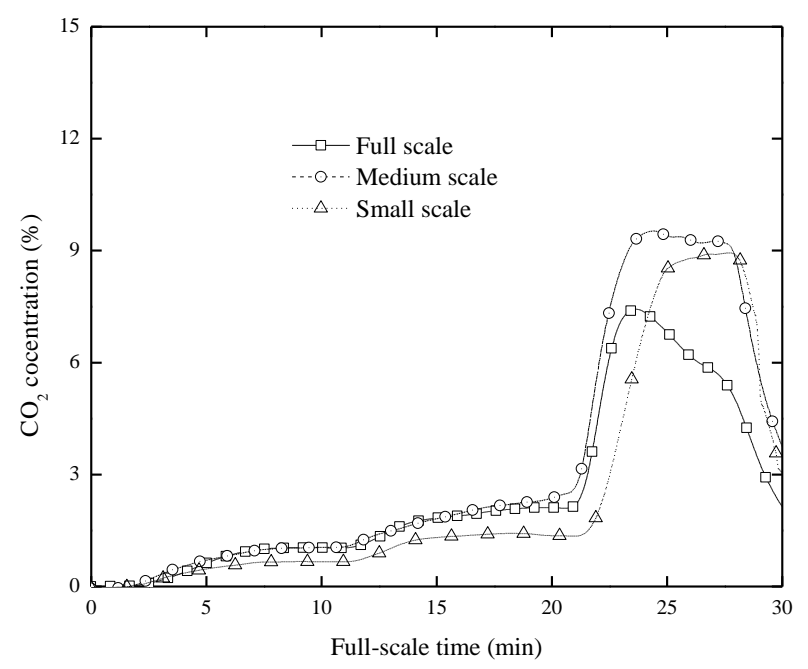

Figure $16 \mathrm{CO}_{2}$ concentration at door for corner fires.

In summary, the gas concentrations are scaled well for both center fires and corner fires. However, for large fires, the oxygen concentrations are slightly overestimated and the $\mathrm{CO} 2$ concentrations are overestimated. The reason could be mainly due to the smoke layer height at the door in model scales are slightly overestimated for large fires.

\subsection{Scaling of incident heat flux}

\subsubsection{Center fires}

Figure 17 shows the measured incident heat fluxes in different scales of center fires. Note that for comparison, both the time and the incident heat fluxes in model scales has been scaled up. It shows that there is very good agreement in different scales.

Before $10 \mathrm{~min}$, the radiation is slightly higher in model scales. The main reason is that during this period the main radiation comes from the flame. Note that the flame normally corresponds to a large emissivity, which results in slightly higher radiation in model scales after the data are scaled up.

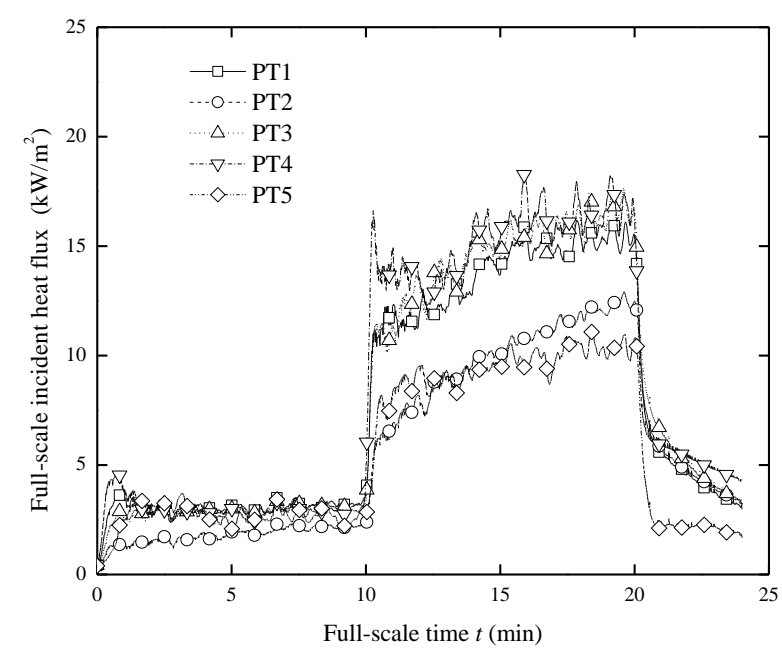

(a) Full scale 


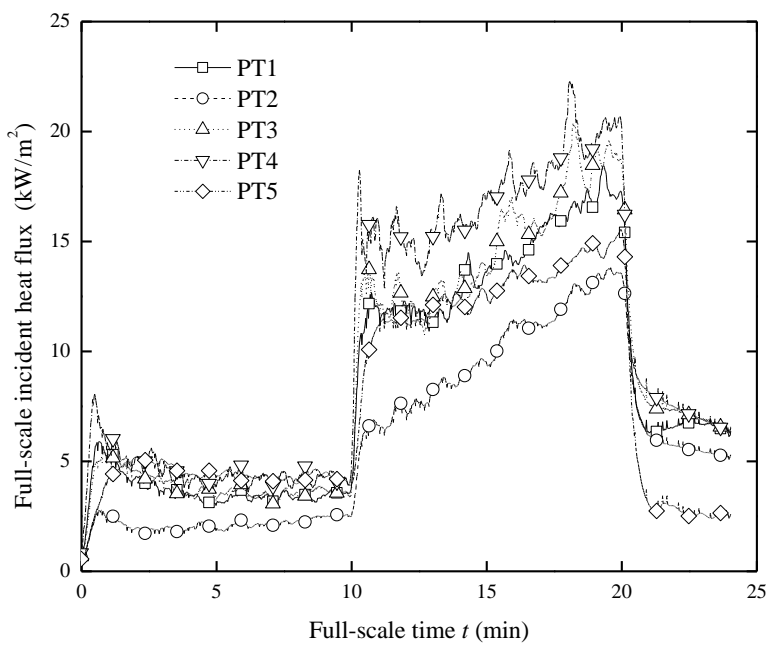

(b) Model scale

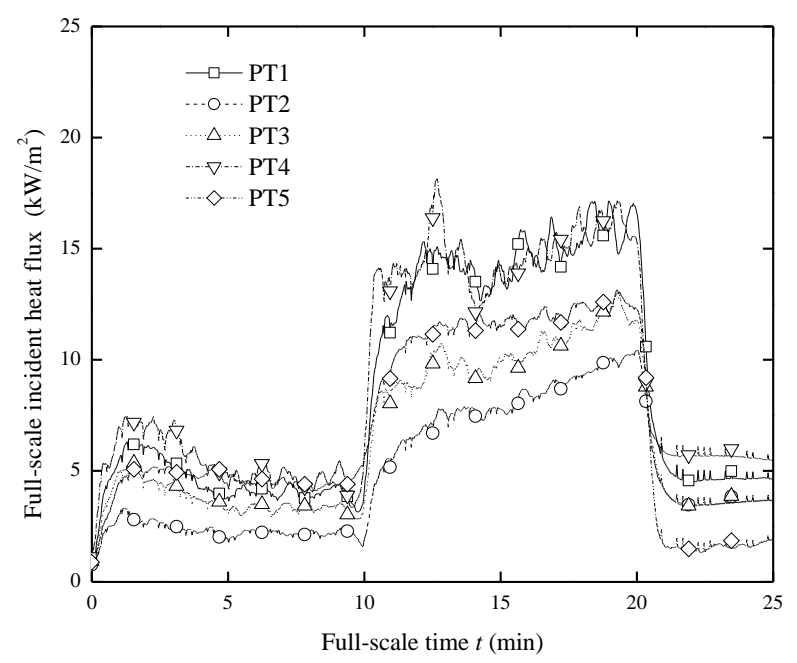

(c) Small scale

Figure 17 Incident heat fluxes in full scale room (center fires, data scaled up).

\subsubsection{Corner fire}

Figure 18 shows the measured incident heat fluxes in different scales of corner fires. Note that for comparison, both the time and the incident heat fluxes in model scales has been scaled up. It shows that the medium scale matches the full scale very well at each stage. The heat fluxes are scaled relatively well in small scale before 20 min, except at PT2 where the heat flux are underestimated after $10 \mathrm{~min}$. After $20 \mathrm{~min}$, the heat fluxes in model scale are generally lower compared to full scale. Part of the reason could be that the convection heat fluxes are overestimated in model scales, especially in small scale with rough walls. Therefore, the overall heat flux imposed on the wall surfaces should be scaled better in small scale than what is shown here. 


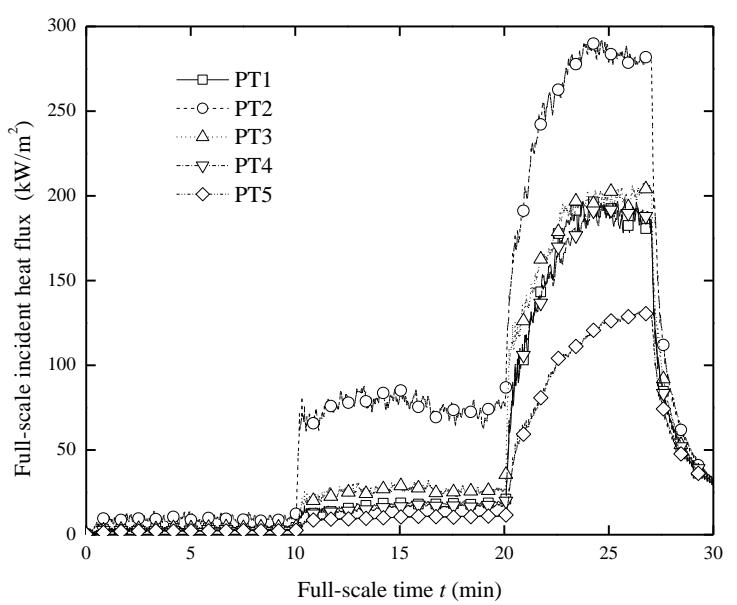

(a) Full scale

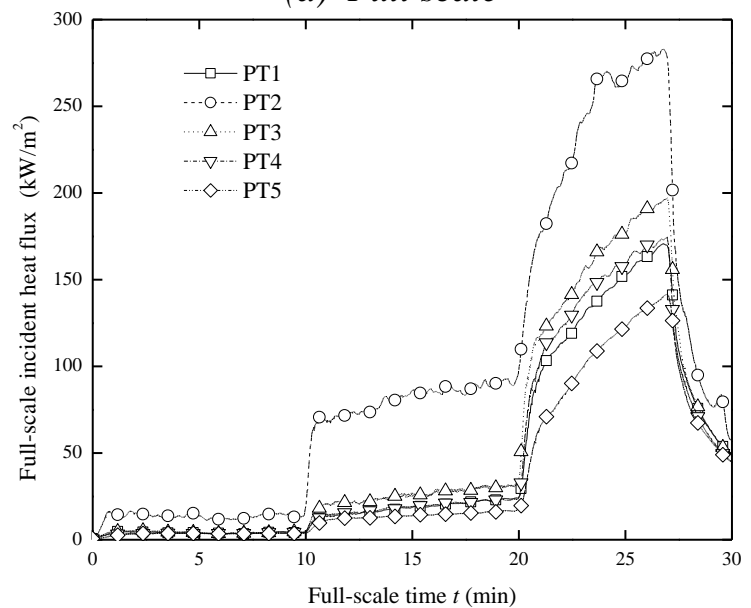

(b) Medium scale

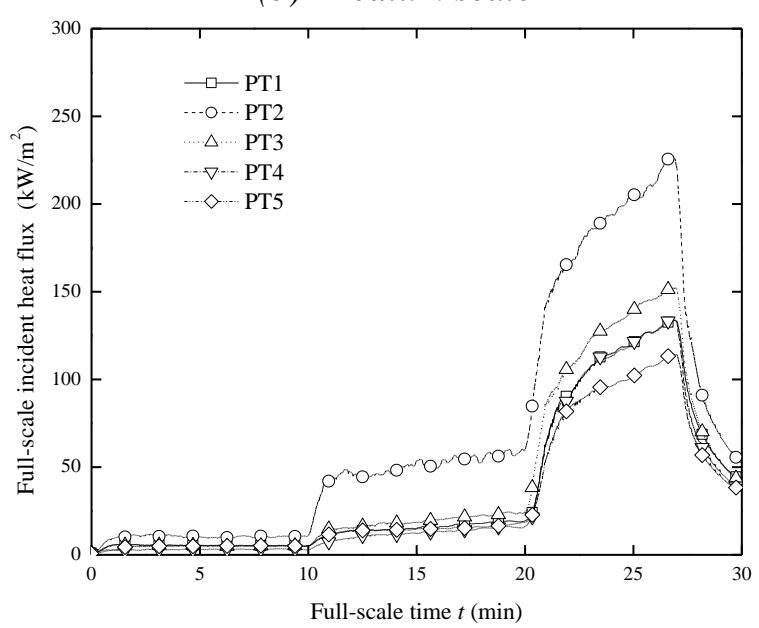

(c) Small scale

Figure 18 Incident heat fluxes in full scale room (corner fire, data scaled up).

Figure 19 and Figure 20 show the comparisons between incident heat fluxes measured in full scale and model scales for center fires and corner fires respectively. Note that all the data have been scaled up to the full scale. Clearly, it can be seen that the incident heat fluxes scales well, especially in medium scales. However, it can also be seen that for small fires with a full-scale heat release rate of $100 \mathrm{~kW}$, the incident heat fluxes are slightly overestimated in model scales, and for large fires with a full-scale heat release rate of 1.2 MW, the incident heat fluxes in small scale are slightly underestimated. 


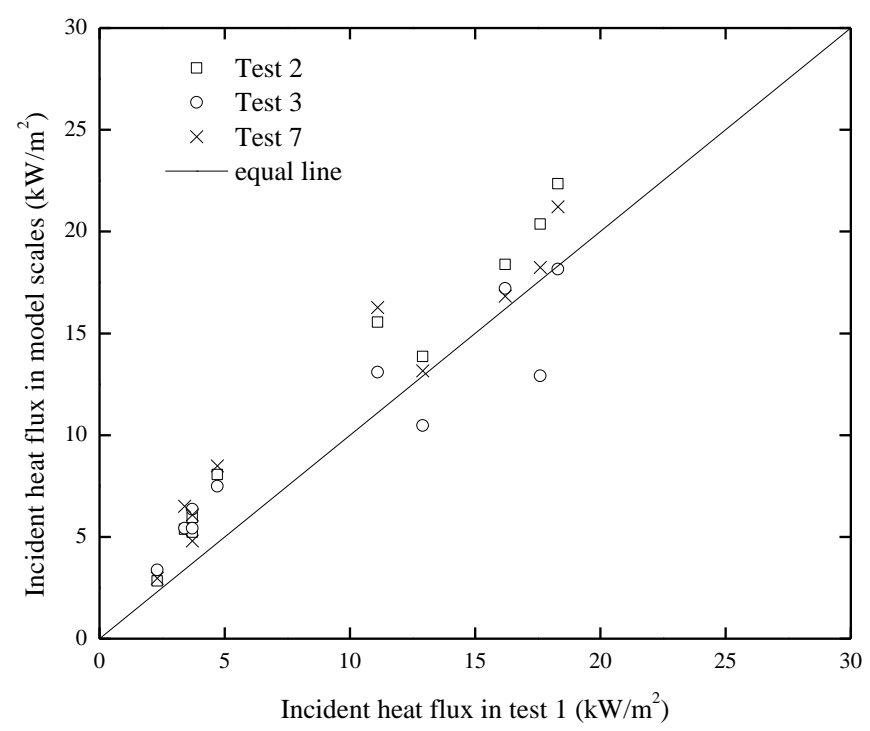

Figure 19 Comparison of incident heat fluxes for center fires (data scaled up).

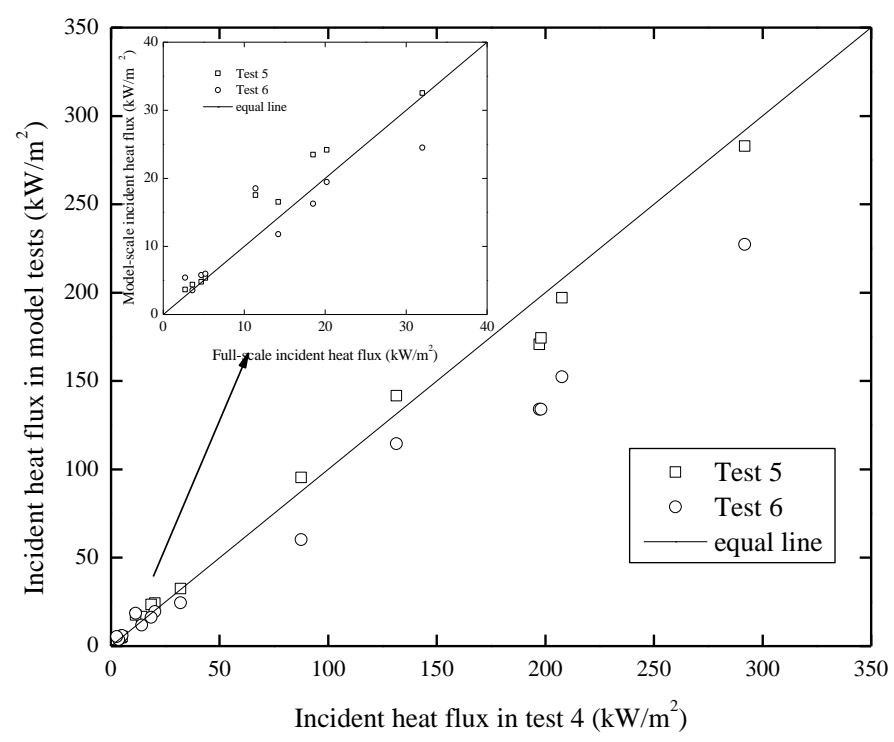

Figure 20 Comparison of incident heat fluxes for corner fires (data scaled up).

In summary, the incident heat flues are scaled very well, especially in medium scale. The incident heat fluxes are only slightly overestimated in model scales for a full-scale heat release rate of $100 \mathrm{~kW}$, and are slightly underestimated in small scale for larger fires.

\subsection{Scaling of internal wall temperature}

\subsubsection{Center fire}

The measured internal wall temperatures at TS1 in different scales for center fires are shown in Figure 21, Figure 22 and Figure 23. Note that for comparison, the time in model scales has been scaled up. $10 \%$ indicates the thermocouple was placed at $10 \%$ of the thickness below the wall surface, and et cetera. Clearly, a perfect correlation can be found 
between the different scales, except that the data measured at $20 \%$ of the thickness below the surface are obviously higher in full scale than model scales, which should be due to that this thermocouple was wrongly positioned.

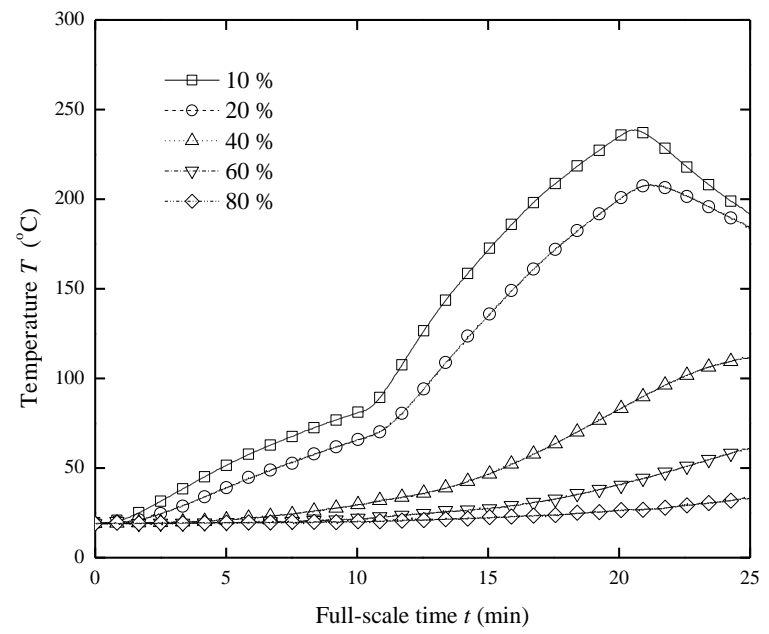

Figure 21 Internal wall temperatures at TS1 in full scale (center fire).

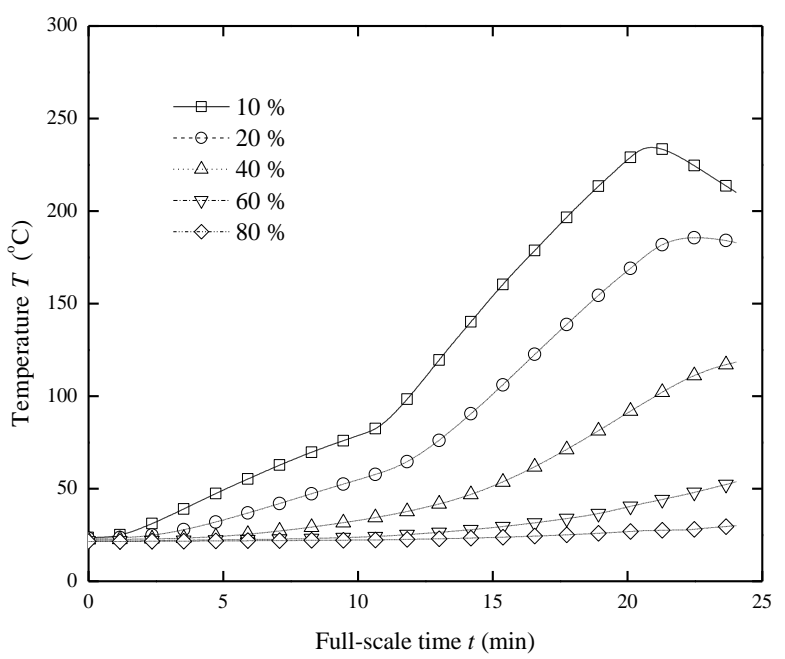

Figure 22 Internal wall temperatures at TS1 in medium scale (center fire).

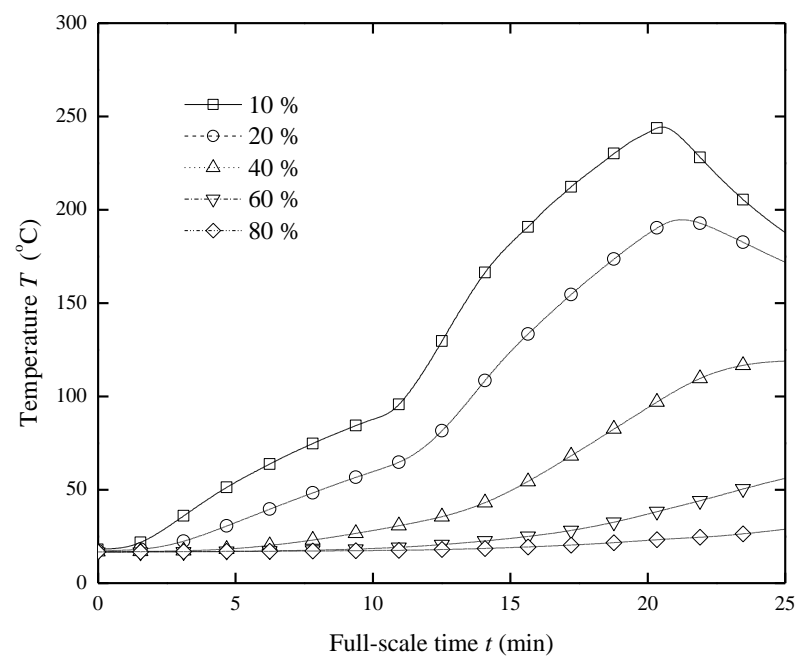

Figure 23 Internal wall temperatures at TS1 in small scale (center fire). 
For clearer comparison, in the following we make a comparison of the internal wall temperatures measured at full-scale time $5 \mathrm{~min}, 10 \mathrm{~min}, 15 \mathrm{~min}, 20 \mathrm{~min}, 23.5 \mathrm{~min}$ and 27 min for center fires. In other words, the time has also been scaled up while picking up the data in mode scale tests.

Figure 24 shows the comparison of the internal wall temperatures in full scale and medium scales of the center fires. Clearly, it shows that there is very good correlation between the full scale and the medium scale. Most data lie closely beside the equal line.

Further, it can be seen that the data scatter slightly. This could be partly due to the error induced by the placement of the thermocouples inside the walls in the tests. Note that the thermocouples are embedded inside the insulating materials and fixed to the exterior frame. Further, the interval distance between the thermocouples are generally very small, that is, the minimum interval is $6 \mathrm{~mm}$ in full scale, $8 \mathrm{~mm}$ in medium scale and $11 \mathrm{~mm}$ in small scale. Therefore, it is difficult to place them perfectly into the right positions, especially in full scale. This also indicates that larger errors could be induced in full scales than in model scales.

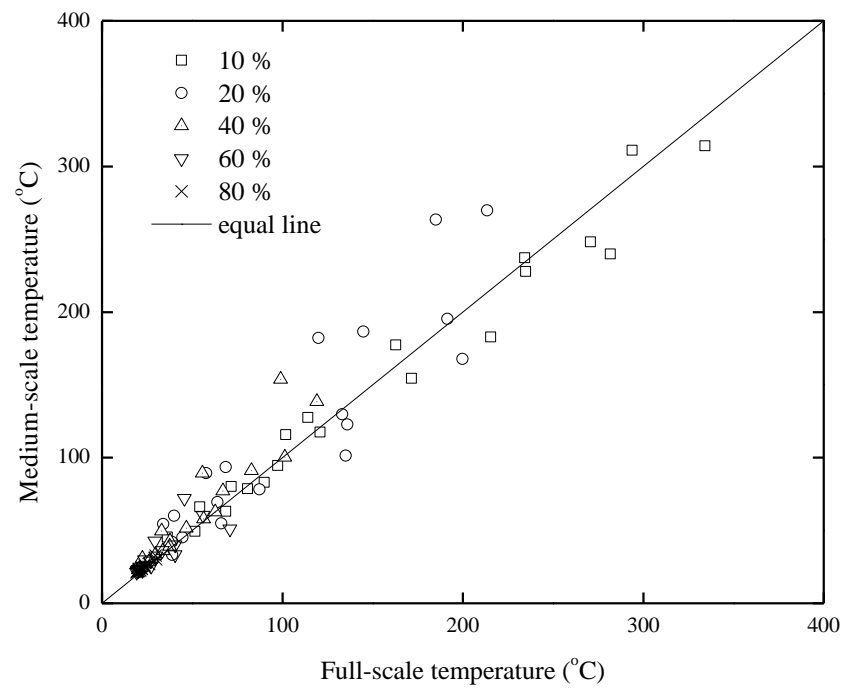

Figure 24 Internal wall temperatures in full scale vs. medium scale (center fires).

Figure 25 shows the comparison of the internal wall temperature in full scale and small scales of the center fires. Clearly, it shows that although the internal wall temperatures in the small scale is slightly lower at some positions, very good correlation can be found between the full scale and the small scale. 


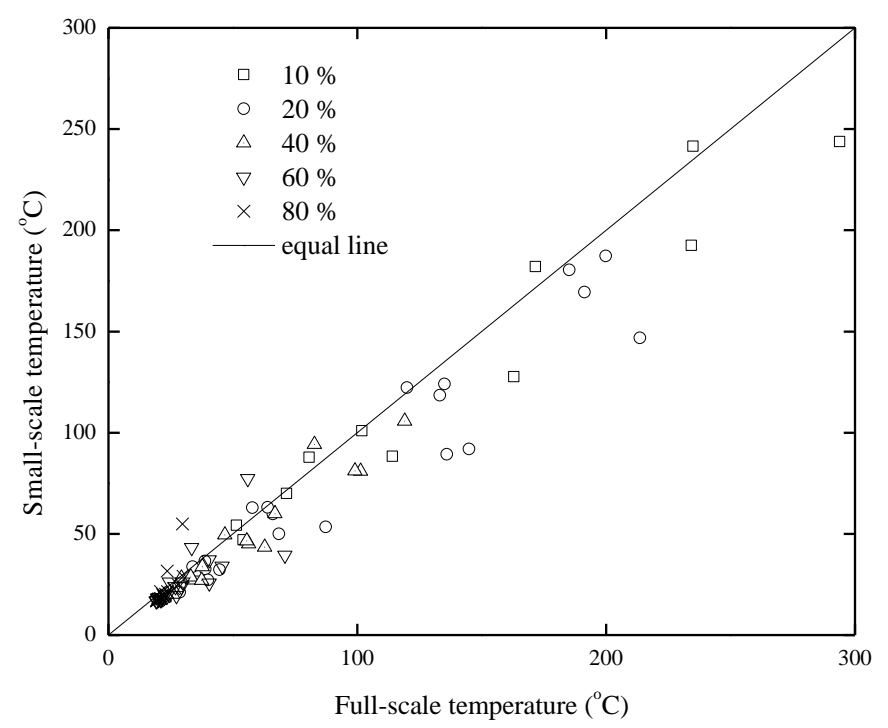

Figure 25 Internal wall temperatures in full scale vs. small scale (center fires).

\subsubsection{Corner fires}

\subsubsection{Small fires}

In this section, the fires before $20 \mathrm{~min}$ at full scale are focused on at first. The corresponding heat release rates are lower than $300 \mathrm{~kW}$ at full scale and these fires called as small fires.

Figure 26 shows the comparison of the internal wall temperature in full scale and medium scale of the corner fires. Note that only tests data before $20 \mathrm{~min}$ are used for comparison. Clearly, it shows that there is very good correlation between the full scale and the medium scale although some data scatters between $100{ }^{\circ} \mathrm{C}$ and $300{ }^{\circ} \mathrm{C}$.

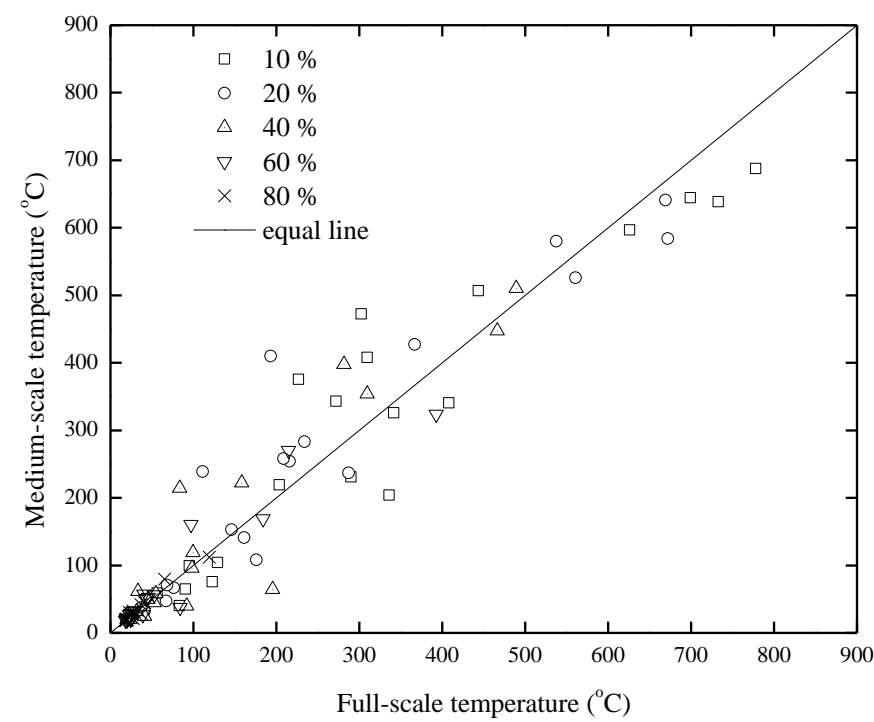

Figure 26 Internal wall temperatures in full scale vs. medium scale (corner fires, within 20min).

Figure 27 shows the comparison of the internal wall temperature in full scale and small scale of the corner fires within 20min. Clearly, it shows that there is good correlation between the full scale and the medium scale although the internal wall temperatures measured in small scale are slightly lower after $600{ }^{\circ} \mathrm{C}$. 


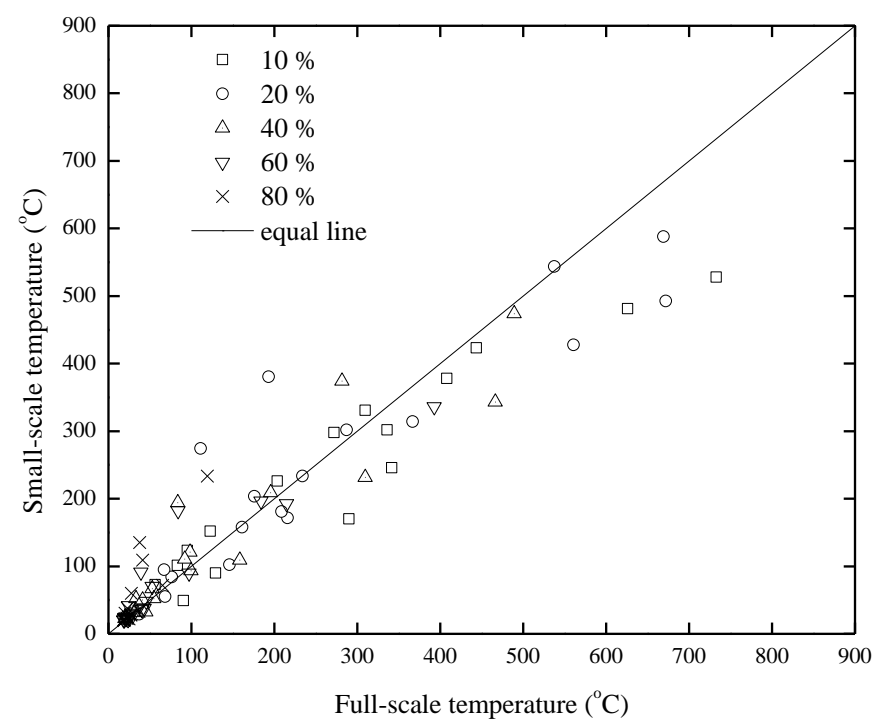

Figure $27 \quad$ Internal wall temperatures in full scale vs. small scale (small corner fires).

\subsubsection{Large fires}

The fires with a full-scale heat release rate of 1.2 MW are considered here, which are called large fires in the report.

Note that in full scale corner fire tests with a heat release rate of $1.2 \mathrm{MW}$, the mineral wool panels in the vicinity of the fire source, started to melt on the surface at around 23 min and some portion of the materials fell down, while they were exposed to gases with high temperatures, see Figure 28. The thicknesses of the insulating materials decreased which resulting in the change of the positions of the thermocouples relative to the wall surfaces. Therefore, the temperature increases significantly after $23 \mathrm{~min}$, which can be seen from Figure D8. The two location significantly affected by this in full scale are TS5 and TS3. These two positions corresponded to high flame temperatures above approximately $1100{ }^{\circ} \mathrm{C}$. Further, in the medium scale test, the panel above the fire source was slightly detached after the test and the measurements at these two positions, i.e. TS5 and TS3, were also affected. Due to these two reasons, the measured data at these two positions for large fires are not used for comparison in the following.

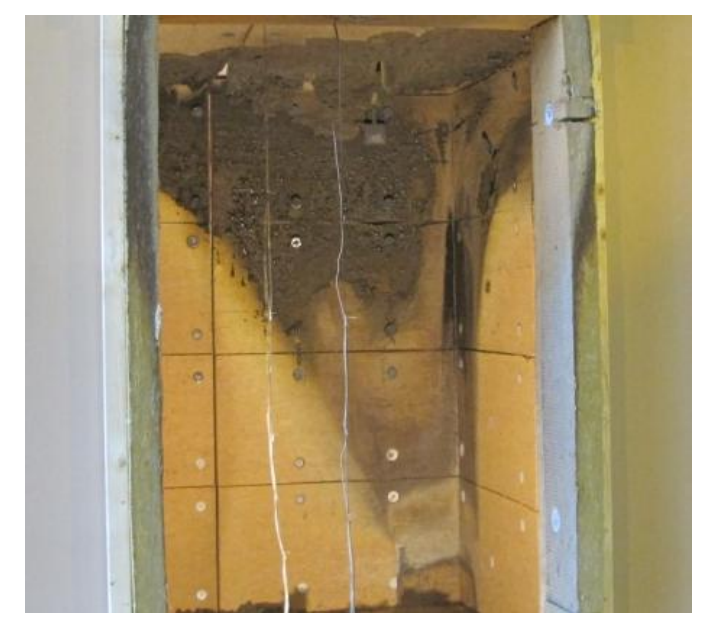

Figure 28 A photo after the full scale test 4.

Figure 29 shows the comparison of the full scale internal wall temperatures to the medium scale values. Figure 30 shows the comparison of the full scale internal wall 
temperatures to the small scale values. Clearly, it shows in Figure 29 that there is good agreement between the full scale and medium scale for internal wall temperatures lower than $800{ }^{\circ} \mathrm{C}$. Further, it shows in Figure 30 that there is good agreement between the full scale and the small scale for internal wall temperatures lower than $600{ }^{\circ} \mathrm{C}$.

However, it can also noticed that the internal wall temperatures are underestimated for temperatures over $800{ }^{\circ} \mathrm{C}$ in medium scale and over $600{ }^{\circ} \mathrm{C}$ in small scale. There are four reasons for this.

Firstly, as discussed previously, the wall materials in the vicinity of the fire source in full scale were melted on the surface and some portion of the materials fell down while exposed to such high temperatures. This also happened in other scales. From the observation of the wall panels after the tests the thickness of the wall panels changed most significantly in full scale, less in medium scale, and very slightly in small scale. Therefore, the internal wall temperatures measured by the thermocouples in full scale are much greater than the values measured in model scales where no such significant change in the thickness occurred.

Secondly, the upper layer gas temperatures in full scale are slightly higher than in medium scale, and the upper layer gas temperatures in small scale are the lowest.

Thirdly, note that the interval distance between the thermocouples are generally very small, that is, the minimum interval is $6 \mathrm{~mm}$ in full scale, $8 \mathrm{~mm}$ in medium scale and 11 $\mathrm{mm}$ in small scale. This indicates that the internal wall temperatures are most sensitive to the changes in the thickness in full scale, less sensitive in medium scale and least in small scale. This could results in the highest internal wall temperatures measured in full scale, higher in medium scale and the lowest in small scale. Further, note that the changes of the positions of the thermocouples have the strongest influence on the thermocouple close to the surface, and much less for the thermocouples deep in the walls. Therefore, the discrepancy between different scales should be much smaller in a deep thermocouple compared to one close to the surface as explained, that is, a much better agreement can be found for low temperatures. This can explain why still good agreement can be found between the full scale and model scales for large fires with gas temperatures below 800 ${ }^{\circ} \mathrm{C}$ in medium scale and below $600{ }^{\circ} \mathrm{C}$ in small scale.

Fourthly, during the large fires, the heat had penetrated the physical thickness of the mineral wools, reached the exterior structure consisting of $13 \mathrm{~mm}$ gypsum boards and the wooden frames. Note that the structures were not scaled. This indicates too much heat could be lost in model scales through the structure after the heat penetrated the insulating materials, which in turn results in a lower internal wall temperatures in model scales.

Despite all this factors which tend to reduce the internal wall temperatures measured in model scales, the scaling of internal wall temperatures in large fires works relatively well. 


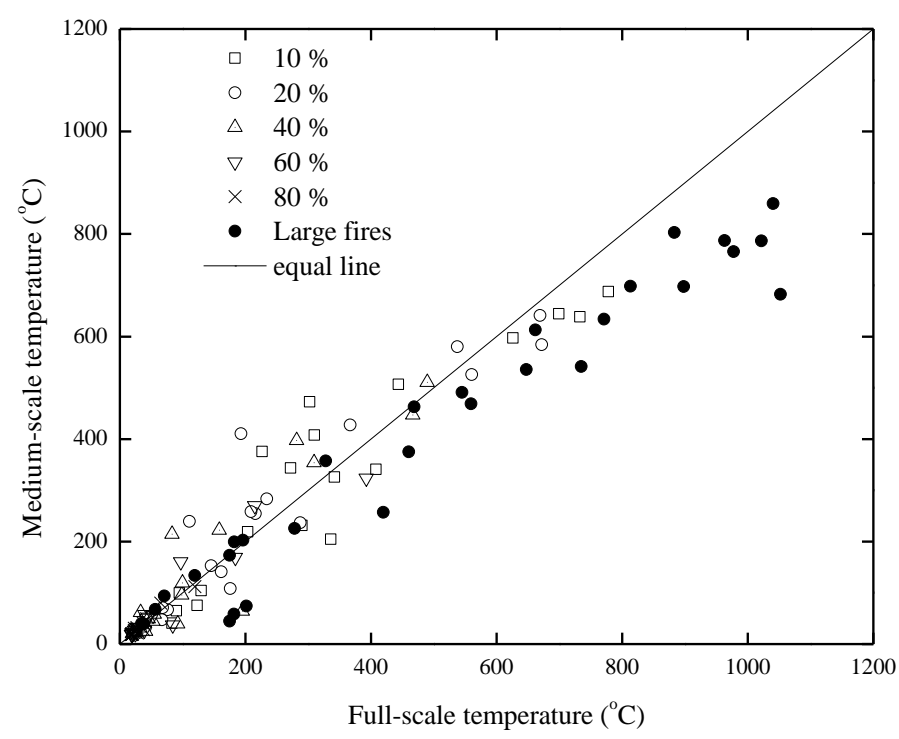

Figure 29 Internal wall temperatures in full scale vs. medium scale (corner fire, including large fires).

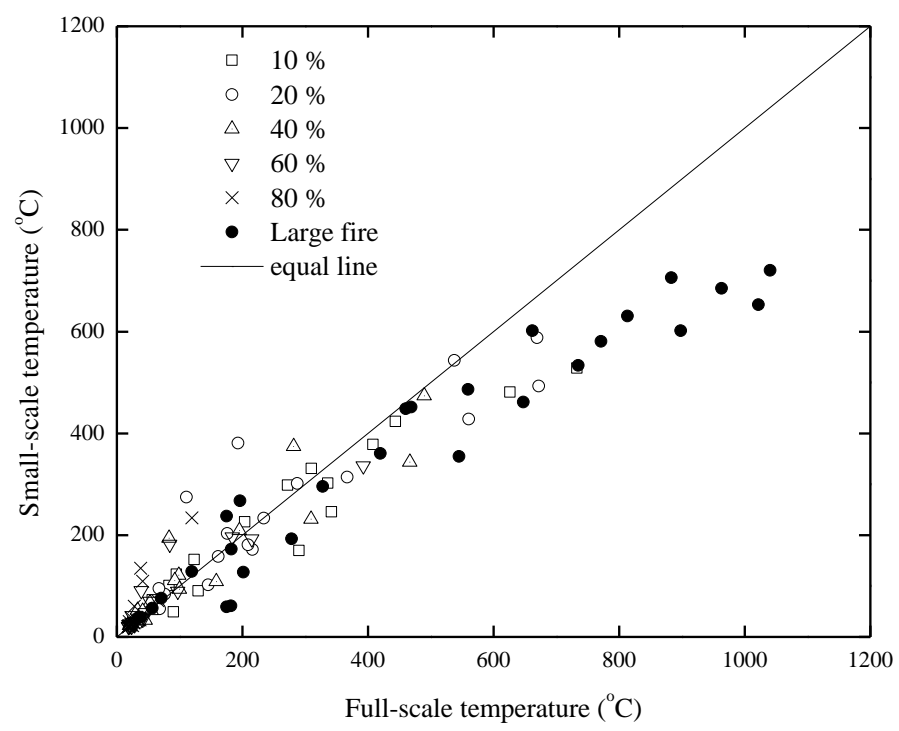

Figure $30 \quad$ Internal wall temperatures in full scale vs. small scale (corner fire, including large fires).

In summary, the scaling of internal wall temperatures for both small center fires and corner fires works very well. For large corner fires, the scaling of internal wall temperatures works relatively well, and slightly better in medium scale compared to small scale.

\subsection{Calculation of ceiling gas temperatures}

In post-flashover enclosure fires, the mass flow rate can be easily obtained due to the insensitivity of the mass flow through the openings, and in turn the gas temperature could be estimated based on the energy equation, assuming the heat released inside the compartment is known. 
In pre-flashover enclosure fires, the mass flow rate through the openings cannot be simply obtained, instead, a numerical solution is required for a quasi-steady fire. This in turn can be used to estimate the gas temperature based on the two-zone model assumption, which should be appropriate for normal enclosure fires. However, simple explicit empirical equation is much easier and more widely used in engineering applications, such as the well-known McCaffrey, Quintiere and Harkleroad (MQH) equation [33], which is expressed as:

$$
\Delta T_{g}=6.85\left(\frac{Q^{2}}{A_{o} \sqrt{H_{o}} h_{k} A_{T}}\right)^{1 / 3}
$$

Before thermal penetration, the conductive heat coefficient:

$$
h_{k}=\left(\frac{k_{s} \rho_{s} c_{s}}{t}\right)^{1 / 2}
$$

and after thermal penetration, the conductive heat coefficient:

$$
h_{k}=\frac{k_{s}}{\delta_{s}}
$$

The penetration time can be esitamted as:

$$
t_{p}=\frac{\delta_{s}^{2}}{4 a}
$$

This gas temperature equation is obtained from dimensional analysis and keeps a form similar to the theoretical implicit equation for the gas temperature. In any case, this equation is empirical.

Delichatsios et al. [34] also proposed a correlation for gas temperatures in enclosure fires based on the adiabatic gas temperature, which is mainly dependent on the heat release rate. This equation has a form similar to the $\mathrm{MQH}$ equation, but the comparison made by Delichatsios et al. [34] showed that the MQH overestimates the gas temperatures.

However, they assumed the smoke layer height as $50 \%$ of the smoke layer height and the calculated adiabatic gas temperature could be as high as $2000 \mathrm{~K}$. Further, the proposed equation is implicit and needs numerical solution and therefore not discussed further here.

Mowrer and Williamsson [35] investigated the gas temperature in fires that were flush against walls or were positioned in a corner. They found the MQH equation still works by by using a correction factor. they recommended that the results from the MQH equation are multiplied by 1.3 for fires flush against walls and by 1.7 for fires in corners.

In the following, the upper layer gas temperature for small fires, i.e. pre-flashover fires, are investigated. The data corresponding to a full-scale heat release rate of $1.2 \mathrm{MW}$, which are related to post-flashover fires, are not taken into account.

\subsubsection{Center fires}

Note that the gas temperatures below the ceiling are dependent on the location relative to the fire source. The gas temperature above the fire is normally higher than at other 
positions. At first, we focuses on the maximum gas temperature measured below the ceiling, i.e. the measured maximum value within thermocouples T1, and T12 to T15. Figure 31 shows the measured maximum ceiling excess gas temperatures for center fires. The full-scale data of the maximum excess gas temperatures for center fires suggest the best fit line:

$$
\Delta T_{g}=6.8\left(\frac{Q^{2}}{A_{o} \sqrt{H_{o}} h_{k} A_{T}}\right)^{1 / 3}
$$

It is shown in Figure 31 that that the data for maximum ceiling excess gas temperature for center fires comply well with the equation which is almost the same as the MQH equation. Note that the maximum excess temperatures were mostly measured right above the fire, i.e. at $\mathrm{T} 1$.

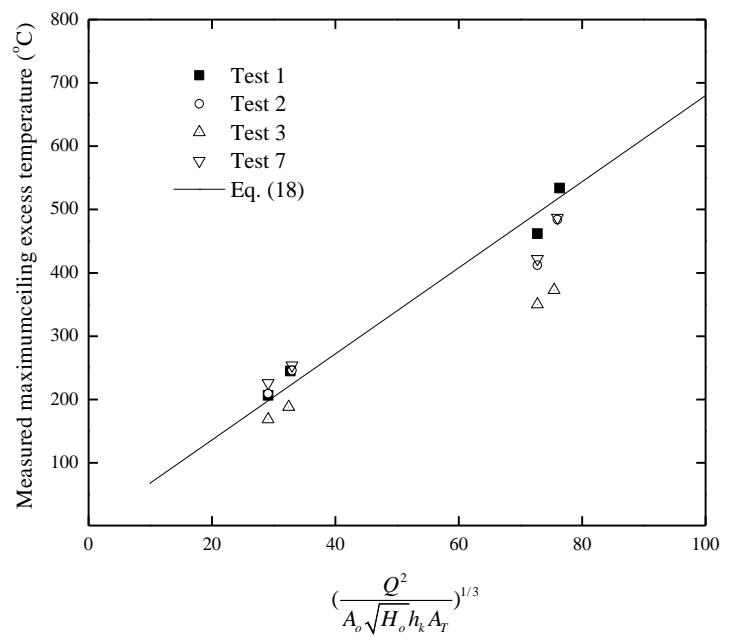

Figure 31 Measured maximum ceiling excess temperature (center fires)

Figure 32 shows the average ceiling excess gas temperature within T12 to T15 for center fires. The average values are representative of the excess temperature of the whole upper layer, and more meaningful in practice compared to the maximum ceiling excess gas temperature discussed above. The full-scale data of the average excess gas temperatures for center fires suggest the best fit line:

$$
\Delta T_{g}=5.7\left(\frac{Q^{2}}{A_{o} \sqrt{H_{o}} h_{k} A_{T}}\right)^{1 / 3}
$$

Comparing the equations for the maximum temperatures and for the average temperatures for center fires show that the average excess gas temperatures are approximately $84 \%$ of the maximum values for center fires. Further, the MQH equation significantly overestimates the average ceiling excess gas temperatures for center fires. 


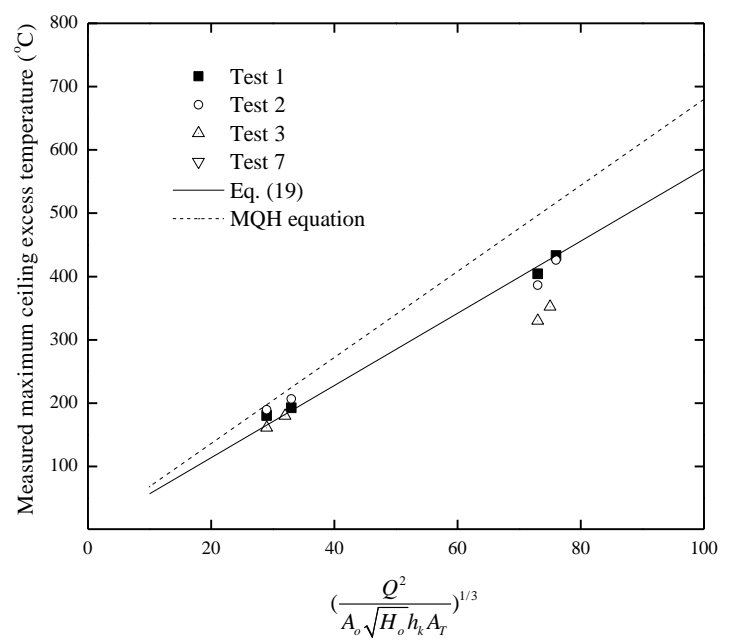

Figure 32 Measured average ceiling excess temperature (center fires)

\subsubsection{Corner fires}

Figure 33 shows the maximum ceiling excess gas temperature for corner fires. The fullscale data of the maximum excess gas temperatures for corner fires suggest the best fit line:

$$
\Delta T_{g}=8.5\left(\frac{Q^{2}}{A_{o} \sqrt{H_{o}} h_{k} A_{T}}\right)^{1 / 3}
$$

Comparing the above equation with the MQH equation shows that the maximum ceiling excess gas temperature for corner fires are much higher than those estimated using the $\mathrm{MQH}$ equation. Note that the maximum excess temperatures were all measured in the vicinity of the fire, i.e. at T14.

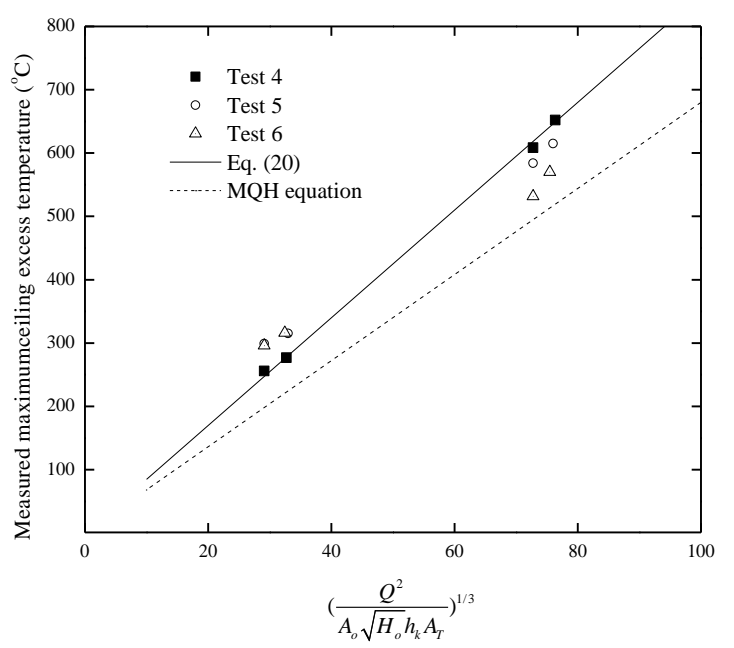

Figure 33 Maximum ceiling excess gas temperature (corner fires)

Figure 34 shows the average ceiling excess gas temperature for center fires. The full-scale data of the average excess gas temperatures for corner fires suggest the best fit line: 


$$
\Delta T_{g}=7.15\left(\frac{Q^{2}}{A_{o} \sqrt{H_{o}} h_{k} A_{T}}\right)^{1 / 3}
$$

Clearly, the average excess gas temperatures are also approximately $84 \%$ of the maximum values for center fires.

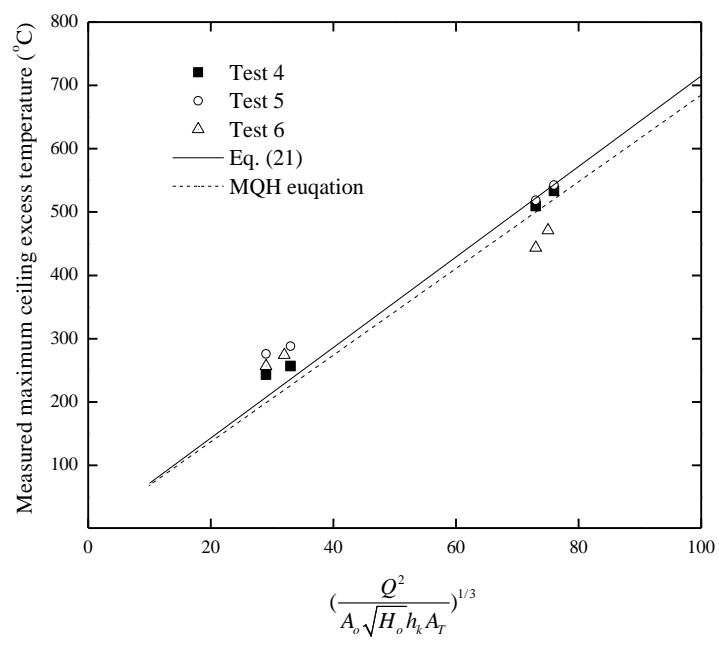

Figure 34 Measured average ceiling excess gas temperature (corner fires)

These results show that both maximum ceiling excess gas temperatures and average ceiling excess gas temperatures for corner fires are higher than those for center fires, and the ratio between corner fires and center fires is 1.25 , for both the maximum and the average ceiling excess gas temperatures. Compared to the coefficient of 1.7 for corner fires proposed by Mowrer and Williamsson [35], the correction factor of 1.25 obtained here is much lower. The correction factor for a fire source detached to the wall is supposed to be lower than this value. This may indicate that the placement of the fire source detached to the corner or the wall does not have strong influence on the gas temperatures. In these cases, the flame tilts towards the opposite side of the wall or corner and the total entrained flow rate by the flame and smoke plume is only slightly lower compared to center fires.

Further, the maximum temperature exists in the vicinity of the fire. The maximum excess gas temperatures are approximately $19 \%$ higher than the average values for both center fires and corner fires.

For center fires, the MQH equation can be used to estimate the maximum ceiling gas temperatures, but it significantly overestimates the average gas temperatures. For corner fires, the MQH equation can be used to approximately estimate the average ceiling gas temperatures, but it significantly underestimates the maximum ceiling gas temperatures.

Based on the above analysis we may propose the following equation for calculation of upper layer excess gas temperatures in enclosure fires:

$$
\Delta T_{g}=5.7 C_{1} C_{2}\left(\frac{Q^{2}}{A_{o} \sqrt{H_{o}} h_{k} A_{T}}\right)^{1 / 3}
$$

where 
and

$$
C_{1}= \begin{cases}1 & \text { for center fires } \\ 1.25 & \text { for corner fires }\end{cases}
$$

$$
C_{2}= \begin{cases}1 & \text { for average temperatures } \\ 1.20 & \text { for maximum temperatures }\end{cases}
$$

Note that the center fires discussed here could be equivalent to any fire in other location in the room except the locations detached to a wall or a corner or placed right in front of one opening. The equation is expected to be valid for gas temperatures below $700{ }^{\circ} \mathrm{C}$ or slightly higher, but not over $1000{ }^{\circ} \mathrm{C}$.

Now we can make an estimation of the transient temperatures in full scale. Figure 35 and Figure 36 show comparisons of measured average temperatures to the calculated values using the proposed equation, i.e. Eq. (22), for test 1 and test 4 respectively. Clearly, the proposed equation correlates well with the tests data. However, the gas temperatures are overestimated during the first $1 \mathrm{~min}$ and slightly underestimated during the first $10 \mathrm{~min}$.

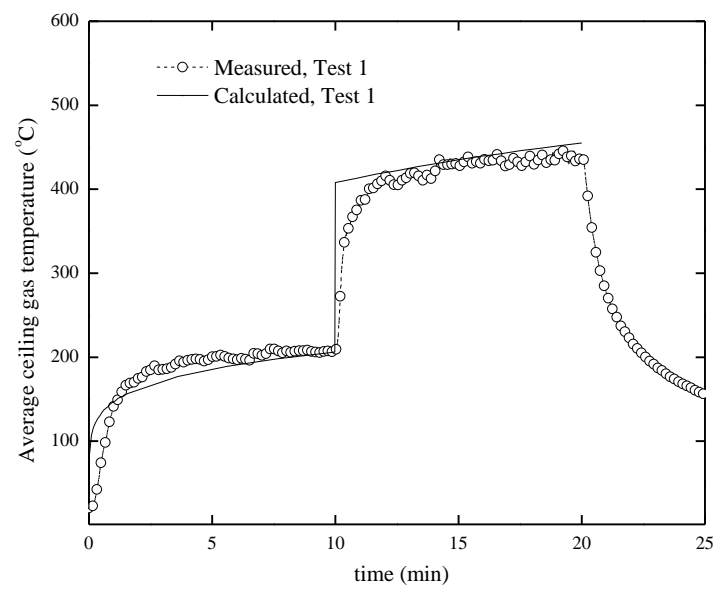

Figure 35 Measured average ceiling excess temperature (center fires test 1)

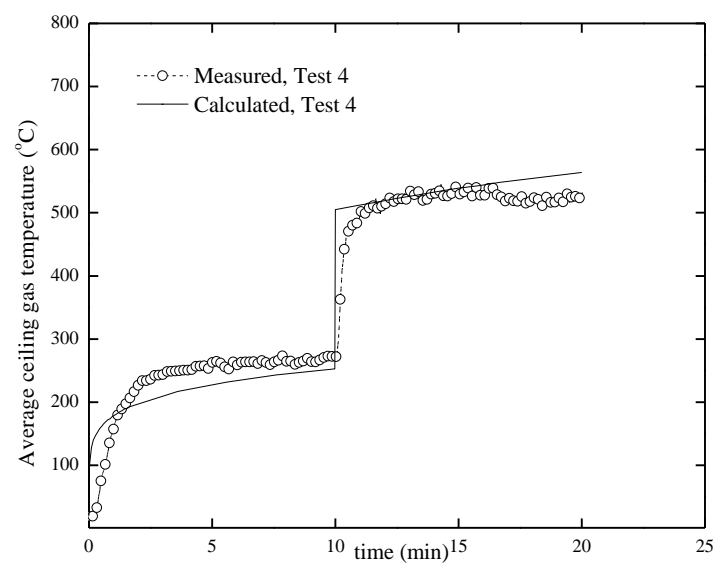

Figure 36 Measured average ceiling excess temperature (corner fires test 4)

It should be kept in mind that the mineral wools are used in the tests. Therefore, the proposed equation could only be used for estimation of gas temperatures in compartments covered by similar insulating materials. 


\subsection{Applications in lightweight constructions}

The above analysis has proved that the internal temperatures of the insulating materials can be scaled well except for the fact that the insulating materials start to melt and fall down while exposed to high temperatures over $1000^{\circ} \mathrm{C}$. For realistic lightweight constructions there are always some panels placed above the insulating materials, which can prevent the collapse of the insulating materials even when it partly performs as thermoplastic materials.

The scaling method proposed in this project can be used to scale the scenarios of lightweight constructions even for large fires if the exterior surfaces of the insulating materials are appropriately covered by a panel. 


\section{$7 \quad$ Conclusions}

The scaling of enclosure fires including internal wall temperatures and heat fluxes is thoroughly analyzed and a method for scaling of internal wall temperatures is proposed. Two series of room fire tests were carried out in three different scales, i.e. full scale (1:2), medium scale (1:2) and small scale (1:3.5), according to the theory. The fire sources were either placed at the center or in the corner of the enclosures. The main focus of these tests was scaling of internal wall temperatures inside the insulating materials. Based on the tests data, the scaling of internal wall temperatures, incident heat fluxes, gas temperatures, gas velocities and gas concentrations is analyzed. Further, the calculation of upper layer gas temperatures in the compartment fires is investigated.

The scaling of internal wall temperatures for both small center fires and corner fires works very well. For large corner fires, the internal wall temperatures are scaled relatively well, and slightly better in medium scale compared to small scale.

The incident heat flues are scaled very well, especially in medium scale. The incident heat fluxes are only slightly overestimated in model scales for a full-scale heat release rate of $100 \mathrm{~kW}$, and are slightly underestimated in small scale for larger fires.

For center fires, the measured upper layer gas temperatures correlate well in different scales, especially in medium scale tests where a perfect match can be found. In small scales, the upper layer gas temperatures are slightly lower, especially at the 2 nd stage. For corner fires, the ceiling gas temperatures are scaled very well before $20 \mathrm{~min}$ but are slightly underestimated in model scales after $20 \mathrm{~min}$ at a heat release rate of $1.2 \mathrm{MW}$ at full scale. The vertical temperature distribution at the center of the room is scaled well for both corner fires and center fires, with the exception that some data points in center fires scattered due to the strong influence of the air flow through the openings on the flame. The vertical temperature distribution at the door is relatively scaled well before $20 \mathrm{~min}$, however, after $20 \mathrm{~min}$, the gas temperatures at the top and at the bottom in model scales are lower in model scales compared to full scale mainly due to relatively higher heat loss through the openings in model scales.

The gas velocities are scaled very well for both center fires and corner fires. The gas concentrations are scaled well for both center fires and corner fires. However, for large fires, the oxygen concentrations are slightly overestimated and the $\mathrm{CO}_{2}$ concentrations are overestimated. The reason could be mainly due to the smoke layer height at the door in model scales are slightly overestimated for large fires.

Both maximum ceiling excess gas temperatures and average ceiling excess gas temperatures for corner fires are higher than those for center fires, and the ratio is 1.25 for both the maximum and the average ceiling excess gas temperatures. The maximum temperature normally exists right above the fire. The maximum excess gas temperatures are approximately $19 \%$ higher than the average values for both center fires and corner fires. For center fires, the MQH equation can be used to estimate the maximum ceiling gas temperatures, but it significantly overestimates the average gas temperatures. For corner fires, the $\mathrm{MQH}$ equation can be used to approximately estimate the average ceiling gas temperatures, but it significantly underestimates the maximum ceiling gas temperatures. A correlation has been proposed to estimate the upper layer gas temperatures in compartments covered by similar insulating materials for both center fires and corner fires. The equation is expected to be valid for gas temperatures below $700{ }^{\circ} \mathrm{C}$ or slightly higher, but not over $1000{ }^{\circ} \mathrm{C}$. Note that the proposed equation could only be used for estimation of upper layer gas temperatures in compartments covered by similar insulating materials. 
This report should raise our concerns on the importance of wall materials in the scaling of enclosure fires. The scaling of the thermal properties of wall materials should be taken into account in model scale fire tests in the future. 


\section{$8 \quad$ References}

1. Heskestad G (1975) Physical Modeling of Fire. Journal of Fire \& Flammability 6:p. $253-273$

2. Quintiere JG (1989) Scaling Applications in Fire Research. Fire Safety Journal 15:3-29

3. Perricone J, Wang M, Quintiere J (2007) Scale Modeling of the Transient Thermal Response of Insulated Structural Frames Exposed to Fire. Fire Technology 44 (2):113136. doi:10.1007/s10694-007-0029-1

4. Croce PA, Xin Y (2005) Scale modeling of quasi-steady wood crib fires in enclosures. Fire Safety Journal Vol. 40:245-266

5. Heskestad G (2002) Scaling the interaction of water sprays and flames. Fire Safety Journal 37:535-548

6. Heskestad G (2003) Extinction of gas and liquid pool fires with water spray. Fire Safety Journal 38:301-317

7. Quintiere J.G., Su G.Y., N. S (2007) Physical scaling for water mist fire suppression a design application. International Journal on Engineering Performance-Based Fire Codes 9 (2):87-108

8. Yu H.Z., Zhou X.Y., Ditch B.D. Experimental validation of Froude-modeling-based physical scaling of water mist cooling of enclosure fires. In: 9th International Symposium on Fire Safety Science (Poster), Karlsruhe, Germany, 21-26 September 2008. IAFSS, pp 553-564

9. Jayaweera T.M., Yu H.Z. (2008) Scaling of fire cooling by water mist under low drop Reynolds number conditions. Fire Safety Journal 43:7

10. Yu H.Z. Physical scaling of water mist suppression of pool fires in enclosures. In, College Park, MD, 2011. 10th International Symposium on Fire Safety Science.

11. Ingason $\mathrm{H}, \mathrm{Li} \mathrm{YZ} \mathrm{(2010)} \mathrm{Model} \mathrm{scale} \mathrm{tunnel} \mathrm{fire} \mathrm{tests} \mathrm{with} \mathrm{longitudinal} \mathrm{ventilation.}$ Fire Safety Journal 45:371-384

12. Ingason $\mathrm{H}, \mathrm{Li} \mathrm{YZ}$ (2011) Model scale tunnel fire tests with point extraction ventilation. Journal of Fire Protection Engineering 21 (1):5-36

13. Ingason H (2007) Model Scale Railcar Fire Tests. Fire Safety Journal 42 (4):271-282

14. Li YZ, Lei B, Ingason H (2010) Study of critical velocity and backlayering length in longitudinally ventilated tunnel fires. Fire Safety Journal 45:361 - 370

15. Li YZ, Lei B, Ingason H (2011) The maximum temperature of buoyancy-driven smoke flow beneath the ceiling in tunnel fires. Fire Safety Journal 46 (4):204-210

16. Li YZ, Ingason H (2012) The maximum ceiling gas temperature in a large tunnel fire. Fire Safety Journal 48:38-48

17. Li YZ, Ingason H (2012) Position of Maximum Ceiling Temperature in a Tunnel Fire. Fire Technology. doi:10.1007/s10694-012-0309-2

18. Li YZ, Ingason H (2010) Maximum Temperature beneath Ceiling in a Tunnel Fire. SP Report 2010:51, SP Technical Research Institute of Sweden,

19. Li YZ, Lei B, Ingason H (2011) Theoretical and Experimental Study of Critical Velocity for Smoke Control in a Tunnel Cross-Passage. Fire Technology. doi:10.1007/s10694-010-0170-0

20. Li YZ, Lei B, Ingason H (2012) Scale modeling and numerical simulation of smoke control for rescue stations in long railway tunnels. Journal of Fire Protection Engineering 22 (2):101-131. doi:10.1177/1042391512445409

21. Li YZ, Ingason H The Fire Growth Rate in a Ventilated Tunnel Fire. In: 10th International Symposium on Fire Safety Science (IAFSS), , Maryland, USA, 2011. pp 347-358. doi:10.3801/iafss.fss.10-347

22. Lönnermark A, Lindström J, Li YZ (2011) Model-scale metro car fire tests. SP Technical research Institute of Sweden, Borås, Sweden

23. Lönnermark A, Lindström J, Li YZ, Claesson A, Kumm M, Ingason H (2012) Fullscale fire tests with a commuter train in a tunnel. SP Technical Research Institute of Sweden, Borås, Sweden 
24. Ingason H (2008) Model scale tunnel tests with water spray. Fire Safety Journal 43 (7):pp 512-528

25. Li YZ, Ingason H (2011) Model scale tunnel fire tests - Automatic sprinklers SP Report 2011:31

26. Hertzberg T, Arvidson M, Axelsson J Large-scale Fire Tests in a Passenger Cabin. In: Fire and Materials 2009 - Proceedings of the 11th International Conference and Exhibition, Fisherman's Wharf, San Fransisco, USA, 26-28 January 2009. Interscience Communications, pp 527-533

27. Hertzberg T (2009) LASS, Lightweight Construction Applications at Sea. SP Report 2009:13

28. Li YZ, Ingason H, Lönnermark A (2013) Correlations in different scales of metro carriage fire tests. SP Report 2013:13

29. Heskestad G (1983) Luminous Heights of Turbulent Diffusion Flames. Fire Safety Journal 5:103-108

30. Ingason H, Wickström U (2007) Measuring incident radiant heat flux using the plate thermometer Fire Safety Journal Vol. 42 (2):161-166

31. Häggkvist A (2009) The Plate Thermometer as a Mean of Calculating Incident Heat Radiation - A practical and theoretical study. Luleå University of Technology,

32. McCaffrey BJ, Heskestad G (1976) Brief Communications: A Robust Bidirectional Low-Velocity Probe for Flame and Fire Application. Combustion and Flame 26:125-127

33. McCaffrey B.J., Quintiere J.G., Harkleroad M.F. (1981) Estimating room fire temperatures and the likelihood of flashover using fire test data correlations. Fire Technology 17 (2):98-119

34. Delichatsios M, Lee YP, Tofilo P (1981) A new correlation for gas temperature inside a burning enclosure. Fire Safety Journal 44 (8):1003-1009

35. Mowrer F, Williamson R (1987) Estimating room temperatures from fires along walls and in corners. Fire Technology 23 (2):133-145. doi:10.1007/bf01040428

36. Quintiere JG Fire behaviour in building compartments. In: Proceedings of the Combustion Institutes, 2002. pp 181-193 


\section{Appendix A - Scaling of internal wall temperatures}

The method of scaling used in the tests is the most widely used Froude scaling. The model is geometrically scaled. According to the Froude scaling, some important parameters should be scaled as shown in Table 1. The scaling of the heat fluxes and internal wall temperatures is analyzed in the following.

\section{A1. Scaling of heat conduction in the walls}

\section{A1.1 Thermally thick materials}

It is well known that the heat conduction normal to the wall surface dominates the heat conduction in the wall. Therefore, only the one dimensional heat conduction equation for the material temperature is analyzed here.

Here we classify the wall materials into two categories: thermally thick materials and thermally thin materials. For a thermally thick material, there is always a temperature gradient inside the materials, even after the thermal penetration, which is considered as being important for us. For a thermally thin material, the temperatures inside are homogeneous. It can be easily known that the thermally thin materials are only special examples of the thermally thick materials. There is no clear distinction between these two types of "materials", but depends on the specific case that is investigated. Generally, metal objects and very thin materials can be considered as thermally thin materials and others are thermally thick materials.

For thermally thick materials, the controlling equation for heat conduction can be written in:

$$
\rho_{s} c_{s} \frac{\partial T_{s}}{\partial t}=k_{s} \frac{\partial^{2} T_{s}}{\partial z^{2}}
$$

and the boundary conditions at $z=0$ and $\mathrm{z}=\delta_{s}$, can be expressed as:

$$
k_{s} \frac{\partial T_{s}}{\partial z}=\dot{q}_{c}^{\prime \prime}+\dot{q}_{r}^{\prime \prime}
$$

The above equations can be made dimensionless by introducing the following normalizing parameters: reference time $t_{o}$, reference temperature $T_{o}$, and reference thickness $\delta_{s}$. The dimensionless variables are denoted by $\left({ }^{\wedge}\right)$.

The controlling equation for heat conduction is normalized to:

$$
\frac{\partial T_{s}}{\partial \hat{t}}=\frac{k_{s} t_{o}}{\rho_{s} c_{s} \delta_{s}^{2}} \frac{\partial^{2} T_{s}}{\partial \hat{z}^{2}}
$$

with the boundary condition:

$$
\frac{\partial T_{s}}{\partial \hat{z}}=\frac{\left(\dot{q}_{c}^{\prime \prime}+\dot{q}_{r}^{\prime \prime}\right) \delta_{s}}{k_{s} T_{o}}
$$

Then we get $\pi$ groups: 


$$
\pi_{1}=\frac{k_{s} t_{o}}{\rho_{s} c_{s} \delta_{s}^{2}}
$$

and

$$
\pi_{2}=\frac{\left(\dot{q}_{c}^{\prime \prime}+\dot{q}_{r}^{\prime \prime}\right) \delta_{s}}{k_{s} T_{o}}
$$

Here we may assume that the convection and radiation heat fluxes are well scaled, that is, $\dot{q}_{c}^{\prime \prime} \propto l^{1 / 2}$ and $\dot{q}_{r}^{\prime \prime} \propto l^{1 / 2}$. Thus Eq. (A5) and Eq. (A6) indicate:

$$
\frac{\rho_{s} c_{s} \delta_{s}^{2}}{k_{s}} \propto l^{1 / 2}
$$

and

$$
\frac{\delta_{s}}{k_{s}} \propto l^{-1 / 2}
$$

Introducing (A8) into (A7) suggests:

$$
\begin{aligned}
& k_{s} \rho_{s} c_{s} \propto l^{3 / 2} \\
& \frac{k_{s}}{\delta_{s}} \propto l^{1 / 2}
\end{aligned}
$$

The wall materials and the noncombustible surface materials should be chosen according to the above two equations. Note that based on the scaling, the wall temperature at depth $\delta$ in full scale generally corresponds to that at a different position in model scale.

We may simply check the obtained scaling laws. At the beginning of an enclosure fire, that is, when the heat has not yet penetrated the walls, the conductive heat fluxes through wall surfaces can be written in:

$$
\dot{q}_{k}^{\prime \prime}=k_{s} \frac{d T_{s}}{d z} \propto \frac{k_{s}\left(T_{w}-T_{i}\right)}{\sqrt{k_{s} t / \rho_{s} c_{s}}}
$$

This indicates

$$
\dot{q}_{k}^{\prime \prime} \propto\left(k_{s} \rho_{s} c_{s}\right)^{1 / 2} l^{-1 / 4}
$$

Note that $k_{s} \rho_{s} c_{s} \propto l^{3 / 2}$, the above equation indicates

$$
\dot{q}_{k}^{\prime \prime} \propto l^{1 / 2}
$$

After a period when the thermal penetration occurs, the conductive heat flux through wall surfaces can be expressed as:

$$
\dot{q}_{k}^{\prime \prime}=\frac{k_{s}}{\delta_{s}}\left(T_{w}-T_{b w}\right)
$$


Note that $k_{s} / \delta_{s} \propto l^{1 / 2}$, the above equation indicates the same results.

Therefore it is clear that the heat conduction in thermally thick materials can be scaled well if Eq. (16) and Eq. (17) are fulfilled in model scale and the wall surface temperature scales well.

\section{A1.2 Thermally thin materials}

For thermally thin materials, e.g. thin wall materials or metal objects, the properties inside the materials can be assumed to be homogeneous, and the controlling equation can be simply expressed as:

$$
\rho_{s} V_{s} c_{s} \frac{\partial T_{s}}{\partial t}=\dot{q}_{n e t}^{\prime \prime} A_{s}
$$

The characteristic parameters are introduced to normalize the above equation:

$$
\frac{\partial T_{s}}{\partial \hat{t}}=\frac{\dot{q}_{n e t}^{\prime \prime} A_{s} t_{o}}{\rho_{s} V_{s} c_{s} T_{o}}
$$

Here we also assume that the convection and radiation heat fluxes follow are scaled well, that is, $\dot{q}_{n e t}^{\prime \prime} \propto l^{1 / 2}$. Thus the above equation indicate:

$$
\rho_{s} V_{s} \propto l^{3}
$$

This suggests that in order to scale the heat conduction in thermally thin materials, the same materials can be used, if the materials are geometrically scaled. In reality, the scaling of the thermally thick materials also fulfills Eq. (A17) assuming the temperatures inside are homogeneous. As discussed previously, a thermally thin material is only one special example of a thermally thick material.

In summary, to scale the heat conduction inside the thermally thick materials, Eqs. (A9) and (A10) need to be preserved while choosing the materials and the wall thickness. For thermally thin materials, the same materials can be used if the materials are geometrically scaled.

\section{A2. Scaling of convection heat transfer}

In an enclosure fire, the key pattern of the convection heat transfer is the forced heat transfer due to the movement of the ceiling jets in the upper layer and also the forced heat transfer in the lower layer due to the movement of fresh air through the openings. The main mechanism should be forced convection heat transfer. For turbulent flow, the convection heat transfer coefficient $h_{c}$ could be correlated with the Nusselt Number and the Prandtl number:

$$
\mathrm{Nu}=\frac{h_{c} l}{k}=0.037 \operatorname{Re}^{4 / 5} \operatorname{Pr}^{1 / 3}
$$

where

$$
\operatorname{Re}=\frac{u l}{v}, \quad \operatorname{Pr}=\frac{v}{a}
$$


The above equations indicate:

$$
\dot{q}_{c}^{\prime \prime} \propto h_{c} \propto l^{1 / 5}
$$

For laminar flow, the convection heat transfer coefficient $h_{c}$ can also be correlated with Nusselt Number and Prandtl number, which can be expressed as:

$$
\mathrm{Nu}=\frac{h_{c} l}{k}=0.66 \operatorname{Re}^{1 / 2} \operatorname{Pr}^{1 / 3}
$$

This means:

$$
\dot{q}_{c}^{\prime \prime} \propto h_{c} \propto l^{-1 / 4}
$$

For a heated wall at lower layer, the heat transfer could be natural convection, and the Nusselt number could be expressed as:

$$
\mathrm{Nu}_{L}=0.678 \mathrm{Ra}_{l}^{1 / 4}\left(\frac{\operatorname{Pr}}{\operatorname{Pr}+0.952}\right)
$$

where the Rayleigh number is defined as:

$$
\mathrm{Ra}_{l}=\frac{g \beta \Delta T l^{3}}{v^{2}} \operatorname{Pr}
$$

Thus we also obtain Eq. (A21).

Comparing Eqs. (A19) and (A21)with Eq. (8) shows that the convection heat transfer is overestimated in model scales, especially for laminar flow.

\section{A3. Scaling of Radiation heat transfer}

The radiation heat transfer coefficient on wall surfaces could be written in a similar form similar to convection heat transfer:

$$
h_{r}=\varepsilon_{g} \sigma\left(T_{g}^{2}+T_{o}^{2}\right)\left(T_{g}+T_{o}\right)
$$

where

$$
\varepsilon_{g}=1-e^{-\kappa_{m} L_{m}}
$$

It is clear that the emissivity of the gas in the model scale gets smaller than in large scale, however, the ratio is difficult to estimate. Here we just make a simple analysis of the optically thick and optically thin cases.

In the optically thick cases, the emissivity is close to unit. Therefore the scaling of radiation heat flux is:

$$
\dot{q}_{r}^{\prime \prime} \propto l^{0}
$$


In the optically thin case, $\kappa L_{e}$ approaches zero. Therefore the scaling of radiation heat flux is:

$$
\dot{q}_{r}^{\prime \prime} \propto \kappa_{m} L_{m} \propto l
$$

Note that the radiation heat flux is the inverse of the radiation resistance $R_{r}$. At the beginning of an enclosure fire, the scenario is optically thin. However, after a few minutes, the scenario could turn to be optically thick. Therefore, the radiation heat flux could be scaled as $l^{a}(0<a<1)$. Note that the scaling of the heat flux should scale as $l^{1 / 2}$. It could be expected that in model scale, the radiation heat flux may be underestimated at the beginning of the fire and overestimated a certain time after ignition. Based on tests data the coefficient $a$ in the optically thick case could be ascertained for some given locations.

The absorption coefficient is mainly depends on the soot yields and smoke volume flow rate. In other words, it depends on the fuel type, ventilation condition and the specific geometries of the room and the burner. In model scales, if the fuels are the same as in full scale, it can be expected that the absorption coefficient is also the same as in full scale, given that the ventilation conditions and specific geometries are well scaled. Therefore, the scaling of radiation heat flux is mainly related to the mean beam length. In engineering applications, the global radiation heat flux could be calculated using a mean beam length, $L_{e}$, which could be estimated by:

$$
L_{m}=3.6 \frac{V_{b}}{A_{b}}
$$

where $V_{b}$ is the volume of the hot gases and $A_{b}$ is its bounding area. The mean beam length in an enclosure fire is mainly related to the smoke depth. Therefore, it varies with the fire sizes. For large flames and sooty smoke, the emissivity always approach to unit and the mean beam length plays an insignificant role in the radiation. However, for small fires, both the absorption coefficient and the smoke depth are small values, and thus it could play a key role in the total emissivity.

We may chose different fuels in model scales to explicitly scale the radiation heat flux in enclosure fires. However, it should be kept in mind that the absorption coefficient is not only related to the fuel type and heat release rate, but also the length scale, the entrainment of the smoke flows, and the combustion conditions, e.g. an under-ventilated fire produces much more soot. In realistic fires, the absorption coefficient is a timedependent variable during a fire. In any case, the scaling of radiation is difficult based on the above analysis. However, in reality, the fire scales the radiation by itself. Note that limited heat is available to be lost by radiation. Therefore in a real fire, the temperature will decrease if the emissivity is too high in model scales. The radiation fraction in the total heat release rate in open fires and enclosure fires have been observed to be around $20 \%$ to $40 \%$. This mainly results from the self-adjustment of the heat radiation. Although the gas temperature could decrease in model scales, the different in the gas temperature between scales could still be insignificant since heat radiation is proportional to the fourth power of gas temperature. In short, the scaling of radiation heat transfer is still expected to be acceptable in model scales. 


\section{A4. Scaling of heat balance in an enclosure}

The total heat released in a lightweight material constructed room could be balanced by the heat loss by smoke flow out through openings, i.e. doors and windows, $Q_{c o n v}$, and the heat loss by conduction into the walls, $Q_{k}$, and the the radiation throught the openings, $Q_{r}$.

$$
Q=Q_{c}+Q_{k}+Q_{r}
$$

\section{A1.1 Heat loss by convection through vents}

The heat loss by convection through the vents can be expressed as:

$$
Q_{c}=\dot{m}_{g} c_{p}\left(T_{g}-T_{o}\right)
$$

In an enclosure fire, the mass flow rate of smoke flow through an opening normally can be written in such a way:

$$
\dot{m}_{g} \propto C_{d} A_{o} \sqrt{\Delta P}
$$

Assuming that the smoke layer can be scaled well and noting that the pressure difference are scaled as the length scale, the smoke mass flow rate through an opening should approximately be scaled as:

$$
\dot{m}_{g} \propto l^{5 / 2}
$$

For a flashover fire, which is probably ventilation controlled, the mass flow rate of the fresh air flowing into the enclosure should equal the smoke mass flow rate out of the opening, both of which can be approximately expressed:

$$
\dot{m}_{\text {air }}=\dot{m}_{g}=0.5 A_{o} \sqrt{H_{o}} \propto l^{5 / 2}
$$

The heat loss by smoke flow out through the openings can be expressed as:

$$
Q_{\text {conv }} \propto l^{5 / 2}
$$

According to this, it is clear that the heat loss by smoke flow out through openings can scale well even for a flashover fire.

\section{A1.2 Heat loss by conduction into the walls}

According to the analyses in Section 2.2.1 and 2.2.2, the convection heat flux and the radiation heat flux cannot be scaled precisely as $l^{1 / 2}$. This definitely will affect the heat conduction inside the wall, since all the heat into the walls comes from the wall surfaces. To analyze the influence of convection and radiation heat transfer on the heat conduction in the walls, the circuit analog of the heat loss to the walls in an enclosure fire is given, as shown in Fig. 1. 


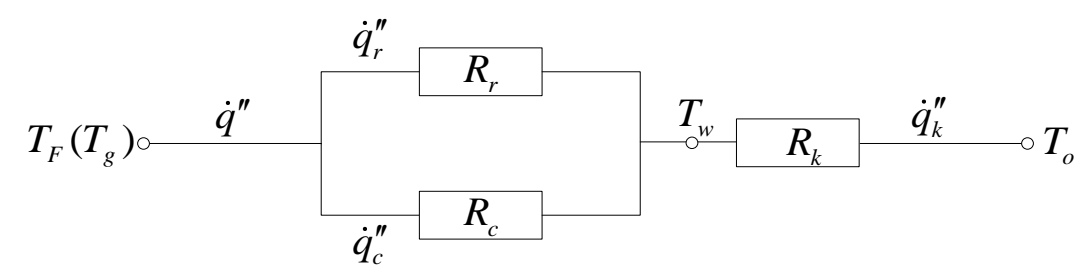

Figure A1. Circuit analog of heat loss to the walls in an enclosure fire

According to Fig. 1, the total heat flux to can be simply expressed as:

$$
\dot{q}^{\prime \prime}=\frac{\left(T_{g}-T_{o}\right)}{R_{t}}=\frac{\left(T_{g}-T_{o}\right)}{\bar{R}+R_{k}} \propto l^{1 / 2}
$$

where

$$
R_{k}=\frac{1}{h_{k}}, R_{c}=\frac{1}{h_{c}}, R_{r}=\frac{1}{h_{r}}, \bar{R}=\frac{R_{r} R_{c}}{R_{r}+R_{c}}
$$

The heat loss by conduction to the walls can also be expressed as:

$$
Q_{k}=h_{t} A_{w}\left(T_{g}-T_{o}\right)
$$

where the total heat transfer coefficient $h_{t}$ is defined as:

$$
\frac{1}{h_{t}}=\frac{1}{h_{c}+h_{r}}+\frac{1}{h_{k}}
$$

The heat flux in model scale should scale as:

$$
\dot{q}^{\prime \prime} \propto \frac{Q}{A} \propto l^{1 / 2}
$$

According to the previous analysis, it is clear that the conductive heat transfer is scaled as $1 / 2$ power of length scale, assume that Eq. (16) and Eq. (17) are fulfilled in model scale and the other parameters are scaled well. However, in practice the heat flux may not scale as $1 / 2$ power of the length scale due to the influence of the scaling of the convection heat flux and the radiation heat flux. This will be discussed in the following.

Note that it is assumed that the radiation heat transfer is directly related to the flame or gas temperature and is proportional to the temperature difference, i.e. has the same form as the convection heat transfer. However, the radiation heat transfer coefficient is not a constant except for a constant radiation temperature. The formation of the equation is only used for a clear expression of the interaction between different modes of heat transfer.

The above equation indicates that the all the heat resistances should be scaled as:

$$
R \propto l^{-1 / 2}
$$

Note that the smaller one between $R_{r}$ and $R_{c}$ dominates the heat transfer to the wall surfaces. Quintiere [36] gave typical ranges for the heat transfer coefficients where $h_{k} \approx$ 
$10-30 \mathrm{~W} / \mathrm{m}^{2} \cdot \mathrm{K}, h_{r} \approx 5-100 \mathrm{~W} / \mathrm{m}^{2} \cdot \mathrm{K}$, and $h_{c} \approx 5-60 \mathrm{~W} / \mathrm{m}^{2} \cdot \mathrm{K}$. For commonly used gypsum board, the conductive heat transfer coefficient is about $28 \mathrm{~W} / \mathrm{m}^{2} \cdot \mathrm{K}$ in half an hour after ignition and $14 \mathrm{~W} / \mathrm{m}^{2} \cdot \mathrm{K}$ in one hour. We can also calculate the radiation heat transfer coefficient using Eq. (36). Assume that the emissivity equals 0.8 and the wall surfaces are bounded by hot gases, the radiation heat transfer coefficient is about 34 $\mathrm{W} / \mathrm{m}^{2} \cdot \mathrm{K}$ for a gas temperature of $500{ }^{\circ} \mathrm{C}$ and $125 \mathrm{~W} / \mathrm{m}^{2} \cdot \mathrm{K}$ for $1000{ }^{\circ} \mathrm{C}$. It is clearly shown that after the gas temperature increases to about $500{ }^{\circ} \mathrm{C}$ the radiation dominates the heat transfer to the wall surface. It can be expected that for a large enclosure fire, the conductive heat transfer dominates the total heat transfer from the hot gases to the surrounding walls during a long period. Especially in this project, the walls are covered by insulating materials, i.e. mineral wools. This means that if the heat conduction scales well, the total or overall heat transfer should also scale well in such cases. In our cases, the wall temperatures between the wall surface $\left(T_{g}\right)$ and the backside $\left(T_{o}\right)$ are the focus.

Note that the total heat transfer corresponds to the heat transfer from the flame and hot gases $\left(T_{g}\right)$ to the penetration boundary inside the wall $\left(T_{o}\right)$. However, the penetration boundary moves deeper into the wall as the time goes by. Therefore, the effective thermal resistance of the wall is not constant but increases with time. For the wall temperatures at a position close to the wall surface (far away from the penetration boundary), the effective thermal resistance of the wall at the beginning of the fire could not dominate the overall heat transfer from the hot gases to the wall, and thus the internal wall temperature could not scale very well. However, as the penetration depth increases with time and the thermal resistance soon becomes the dominant term in the overall heat transfer from hot gases to the internal walls. In such cases, the internal wall temperatures should scale well.

\section{A1.3 Heat loss by radiation through the vents}

The heat loss by radiation through the vents can be written in:

$$
Q_{r}=\phi \varepsilon \sigma A_{o}\left(T_{g}^{4}-T_{o}^{4}\right)
$$

where $\phi$ is the ratio of the effective radiation area to the area of the opening, which could be approximately estimated based on the smoke layer depth at the opening.

For enclosures with small openings, the heat loss by radiation through the vents can be ignored, compared to the others. However, for enclosures with large openings, the heat loss by radiation through the vents should be taken into account.

The heat loss by radiation through the vents scales the same as the radiation heat flux. Its effect on the entire heat balance mainly depends on the vents area. In our cases, the heat loss by radiation through the vents is not so important.

\section{A5. Short summary}

Based on the above analysis, the scaling of the heat fluxes is given, as shown in Table A1. Note that at a certain time after ignition, especially for a sooty fire, the main thermal resistance of the heat transfer from the hot gases to the backside wall comes from the wall materials. Note that the materials used in the tests are related to thermally thick materials. It can be expected that the heat conduction and the temperature inside these wall materials can be scaled well if Eq. (A9) and Eq. (A10) are fulfilled in model scale.

In summary, it is known that a large amount of heat released in an enclosure fire is taken away by smoke flow out through openings, which is normally called the convective heat release rate. The fraction of the convective heat release rate in the total heat release rate is normally in a range of $60 \%$ to $80 \%$. This is the main reason why the simplified Froude 
scaling can scale the fire scenario well even in model scales even when all the heat fluxes are explicitly scaled.

Table A1 Extra list of scaling correlations for heat fluxes.

\begin{tabular}{lcc}
\hline \multicolumn{1}{c}{ Type of unit } & Scaling & $\begin{array}{c}\text { Equation } \\
\text { number }\end{array}$ \\
\hline Thermally thick materials & $\left(k_{s} \rho_{s} c_{s}\right)_{M} /\left(k_{s} \rho_{s} c_{s}\right)_{F} \propto\left(l_{M} / l_{F}\right)^{3 / 2}$ & $(A 41)$ \\
Thermally thin materials & $\left(\frac{k_{s}}{\delta_{s}}\right)_{M} /\left(\frac{k_{s}}{\delta_{s}}\right)_{F} \propto\left(l_{M} / l_{F}\right)^{1 / 2}$ & $(A 42)$ \\
Conductive heat flux $(\mathrm{kW})$ & $\rho_{s} V_{s} \propto l^{3}$ & $(A 43)$ \\
Radiation heat flux & $\dot{q}_{c o n d, M}^{\prime \prime} / \dot{q}_{c o n d, F}^{\prime \prime}=\left(l_{M} / l_{F}\right)^{1 / 2}$ & $(A 44)$ \\
Convection heat flux & $\dot{q}_{r, M}^{\prime \prime} / \dot{q}_{r, F}^{\prime \prime}=\left(l_{M} / l_{F}\right)^{a}(0 \leq a \leq 1)$ & $(A 45)$ \\
\hline
\end{tabular}




\section{Appendix B - Layout of instrumentation in model scales}

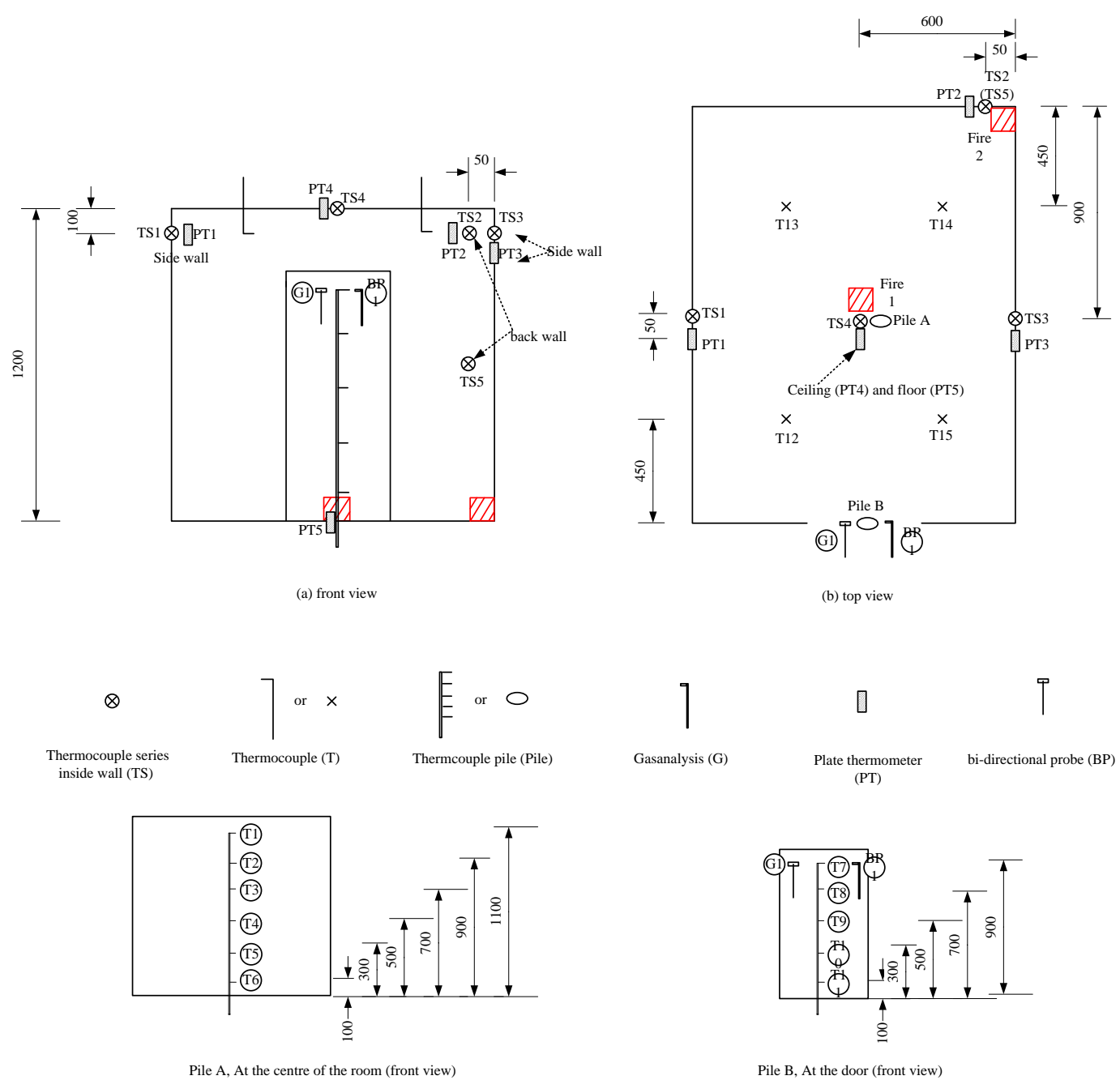

Figure B-1. Layout of instrumentation in 1:2 model tests. 


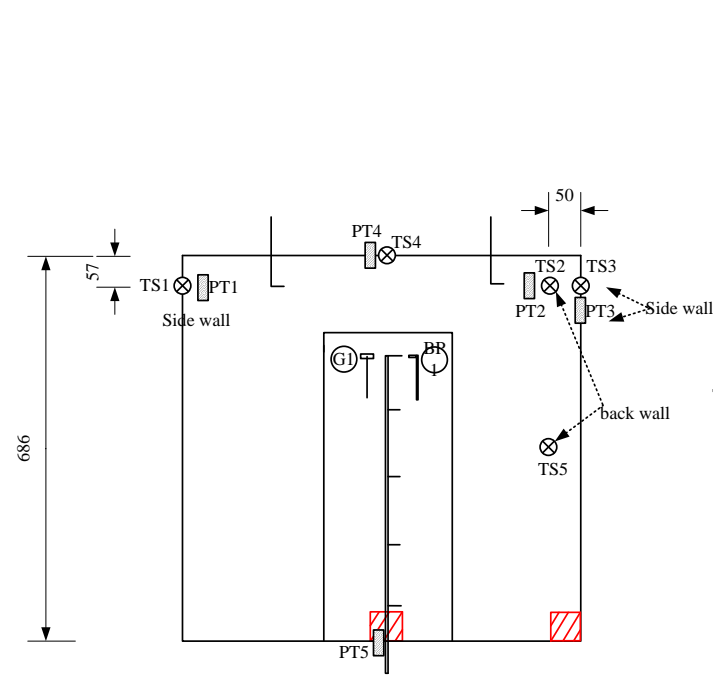

(a) front view

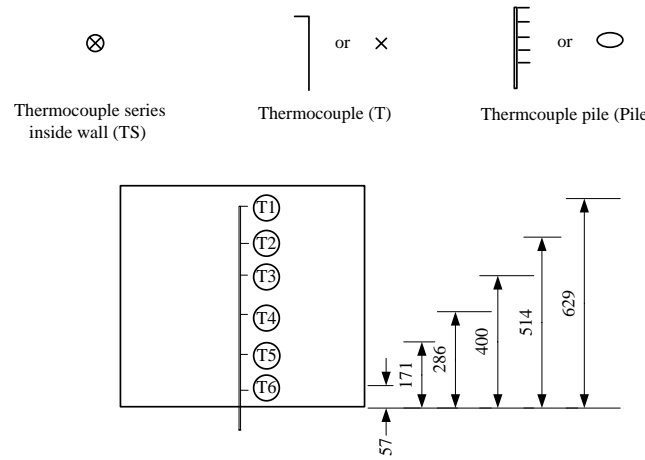

Pile A, At the centre of the room (front view)

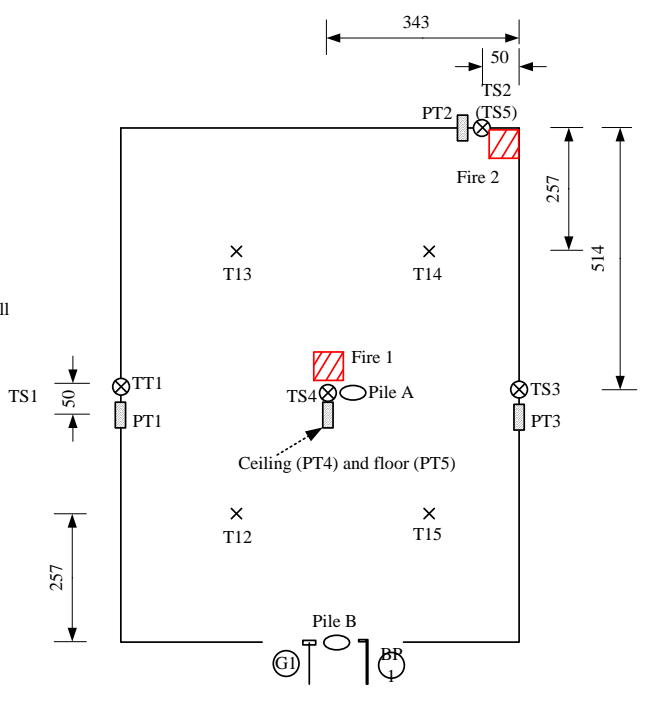

(b) top view

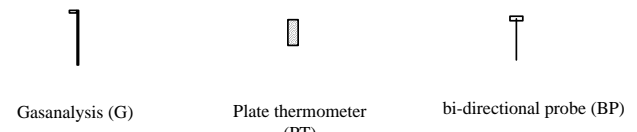

Pile B, At the door (front view)

Figure B-2. Layout of instrumentation in 1:3.5 model tests. 


\section{Appendix C - Photos of the tests}

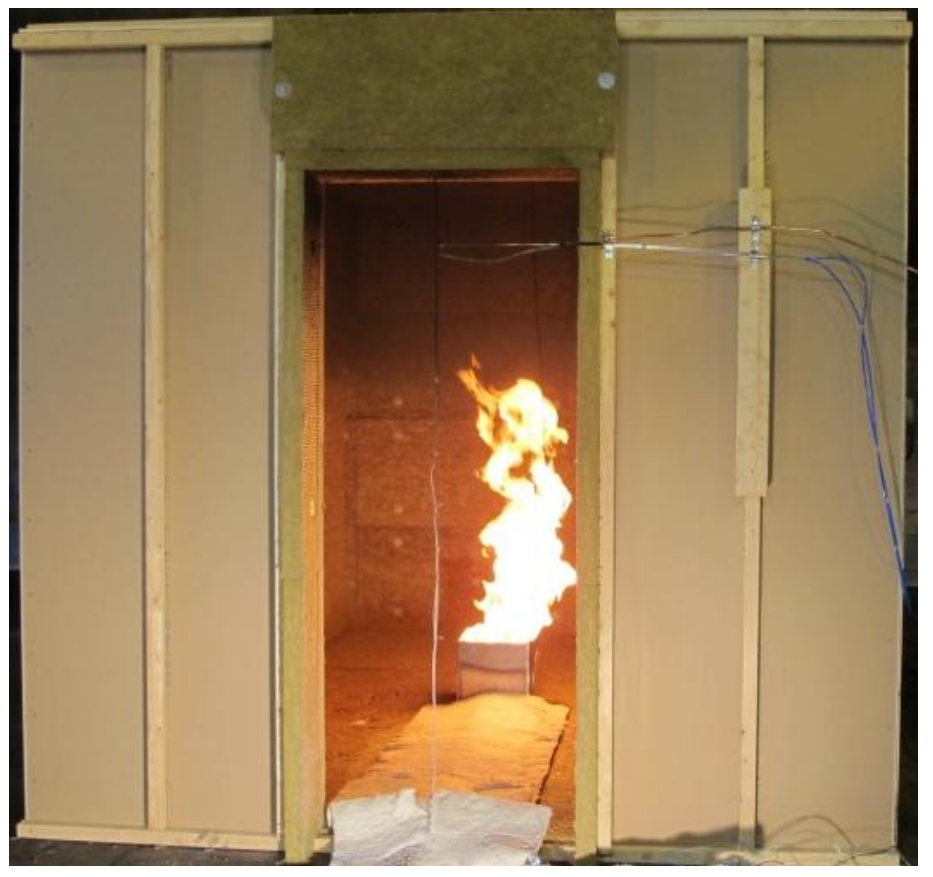

(a) First stage

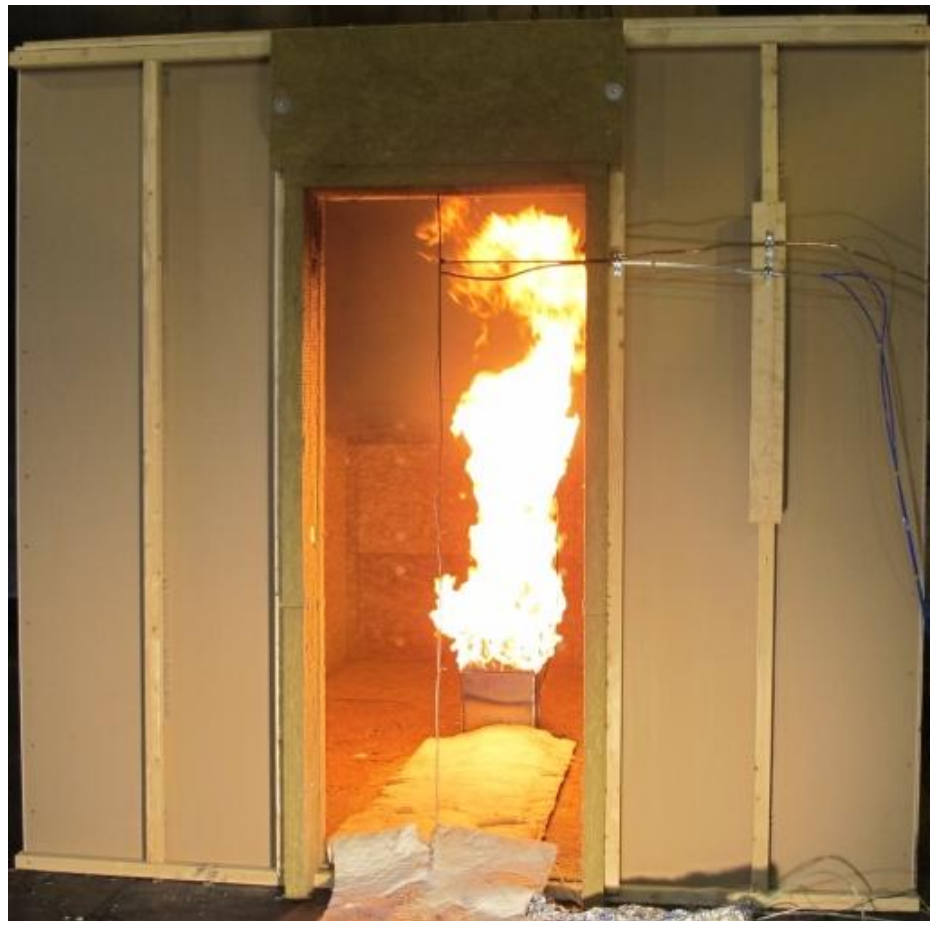

(b) Second stage

Figure C-1. Photos in full scale test 1. 


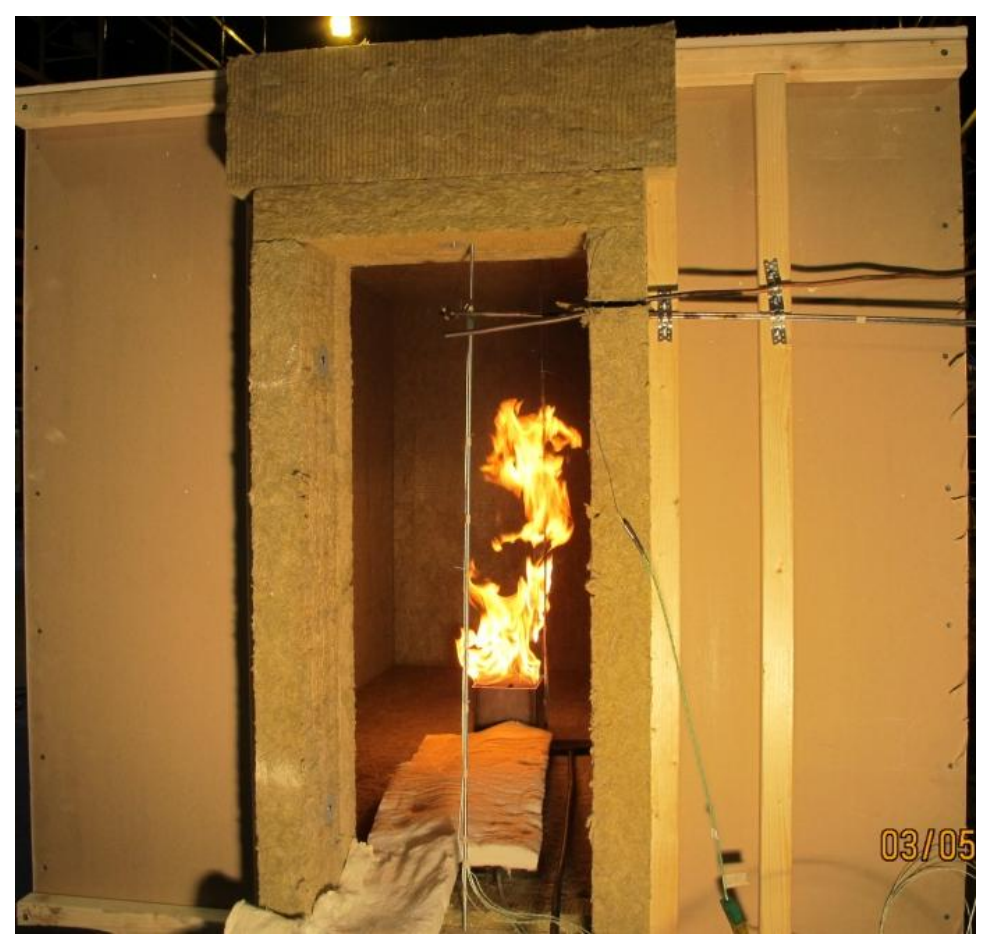

(a) First stage

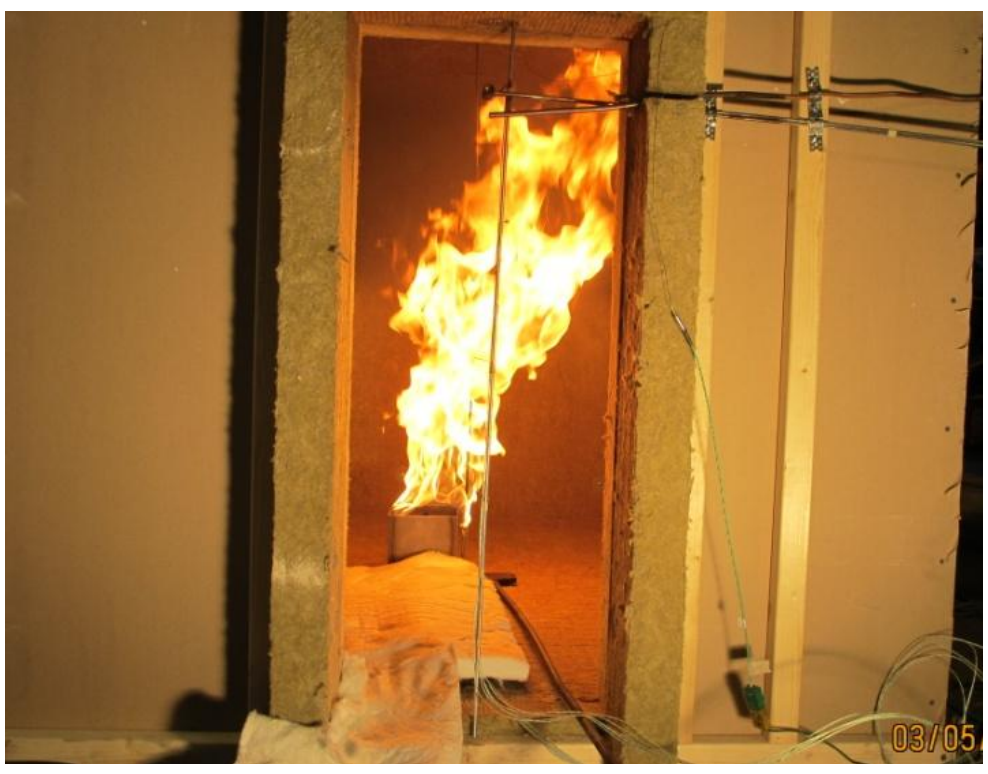

(b) Second stage

Figure C-2. Photos in medium scale test 2. 


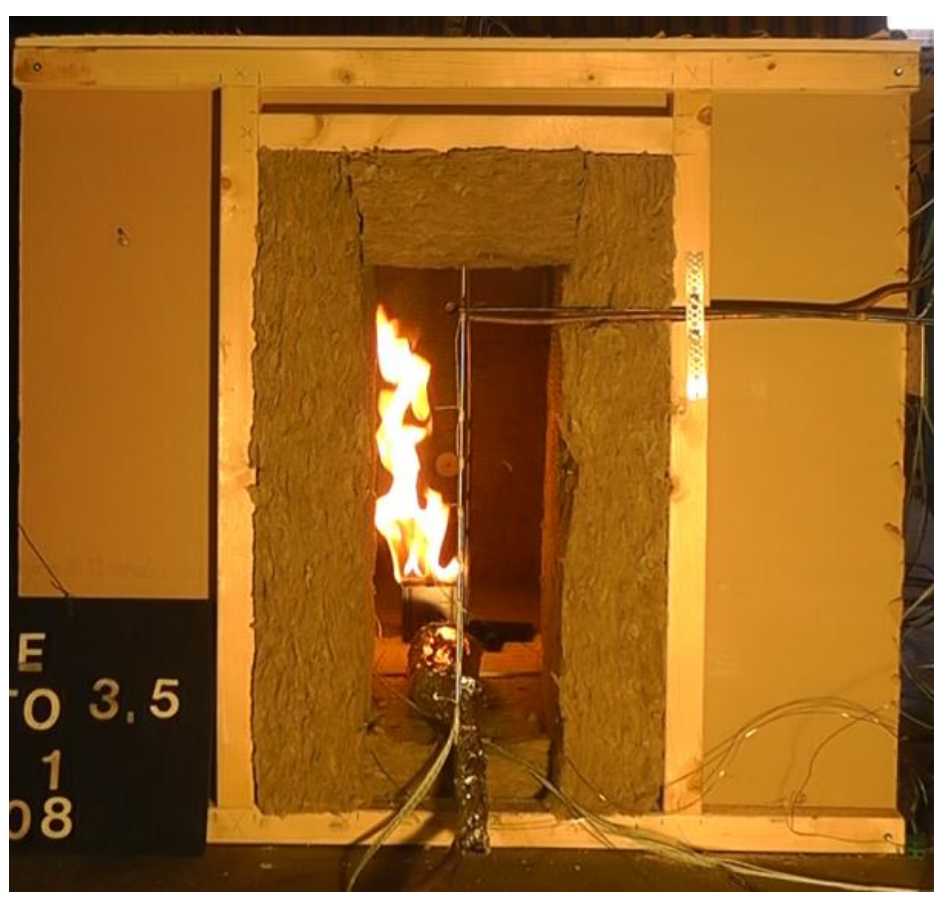

(a) First stage

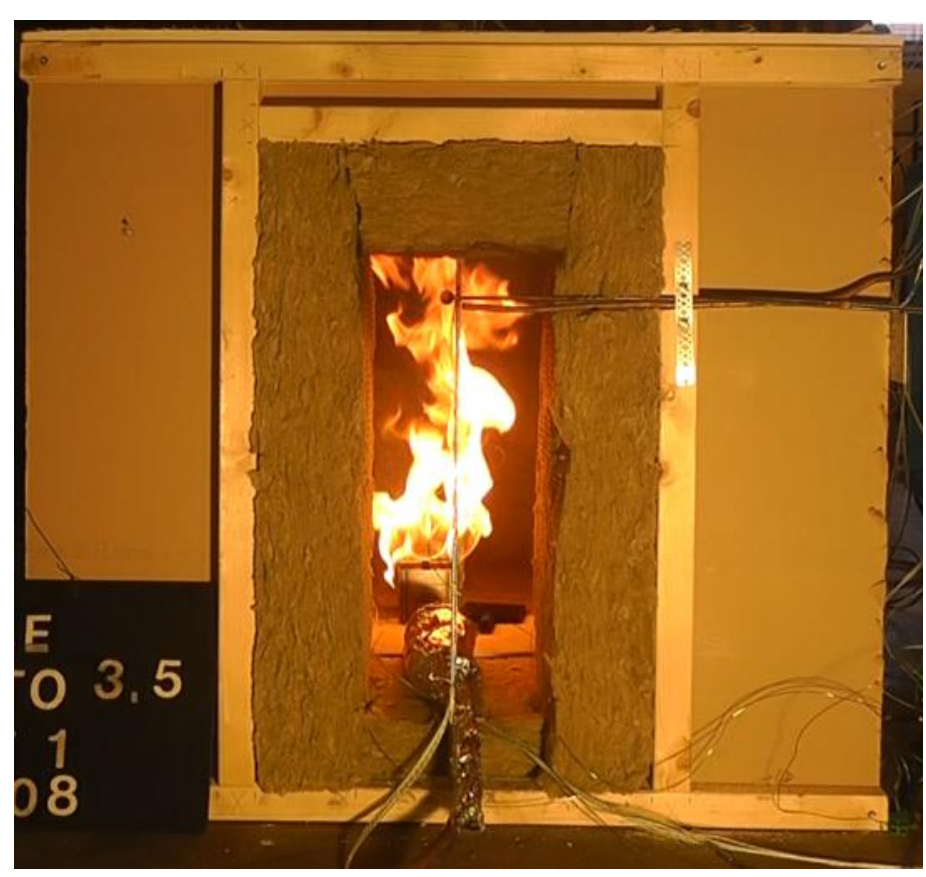

(b) Second stage

Figure C-3. Photos in medium scale test 3. 


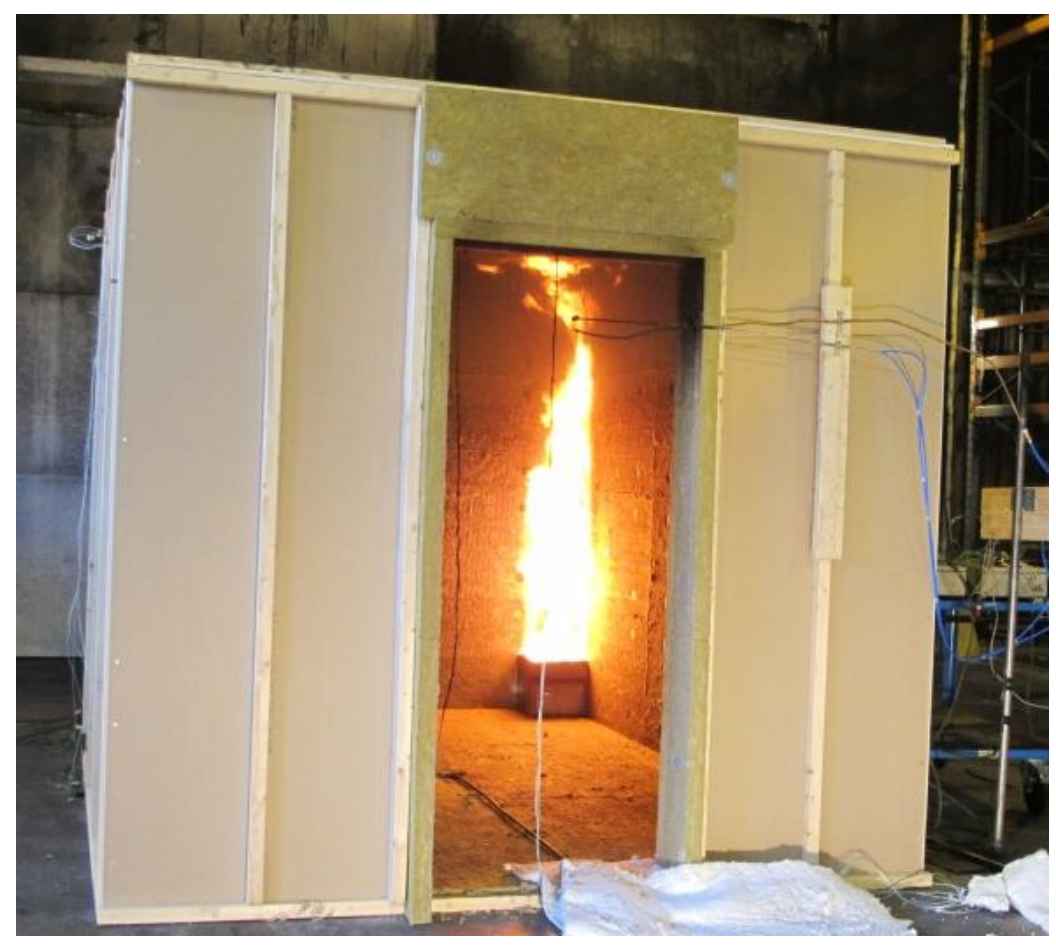

(a) First stage

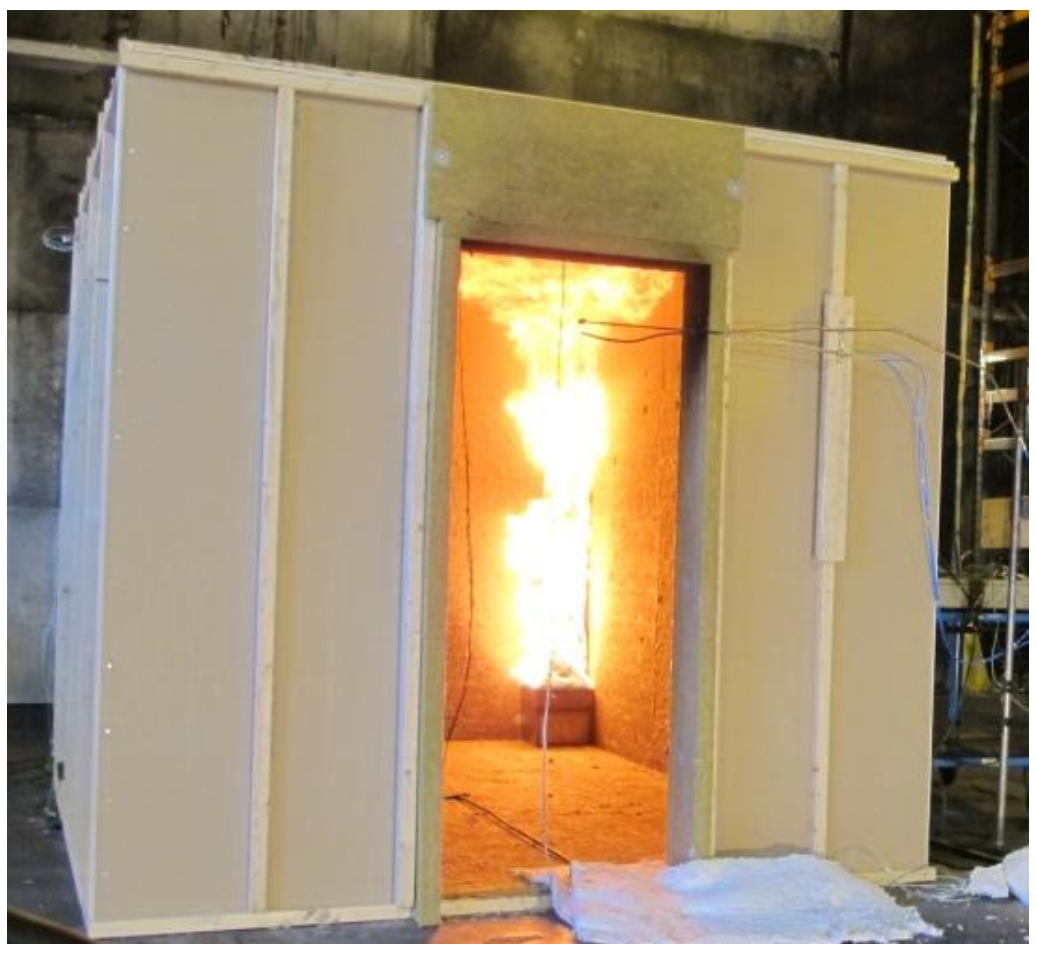

(b) Second stage 

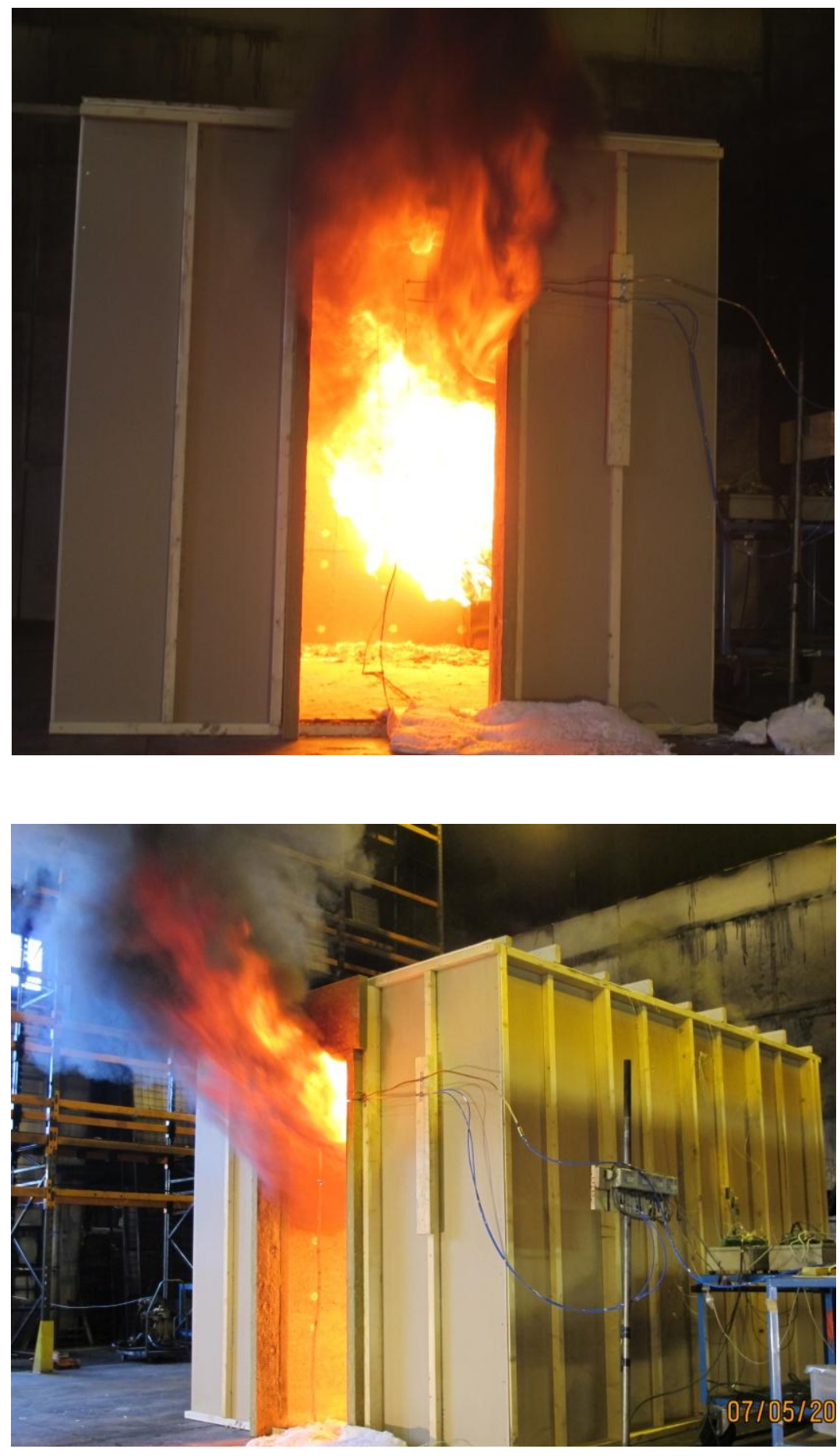

(c) Third stage

Figure C-4. Photos in full scale test 4. 


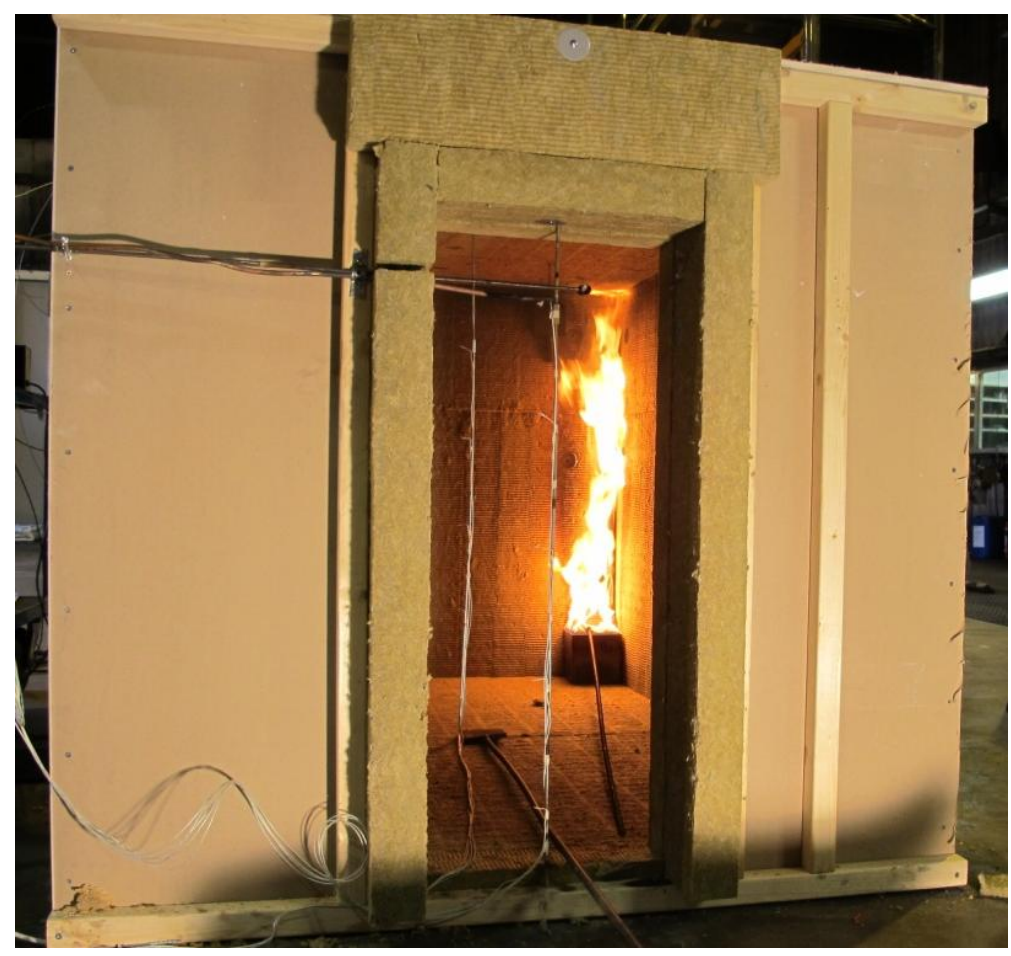

(a) First stage

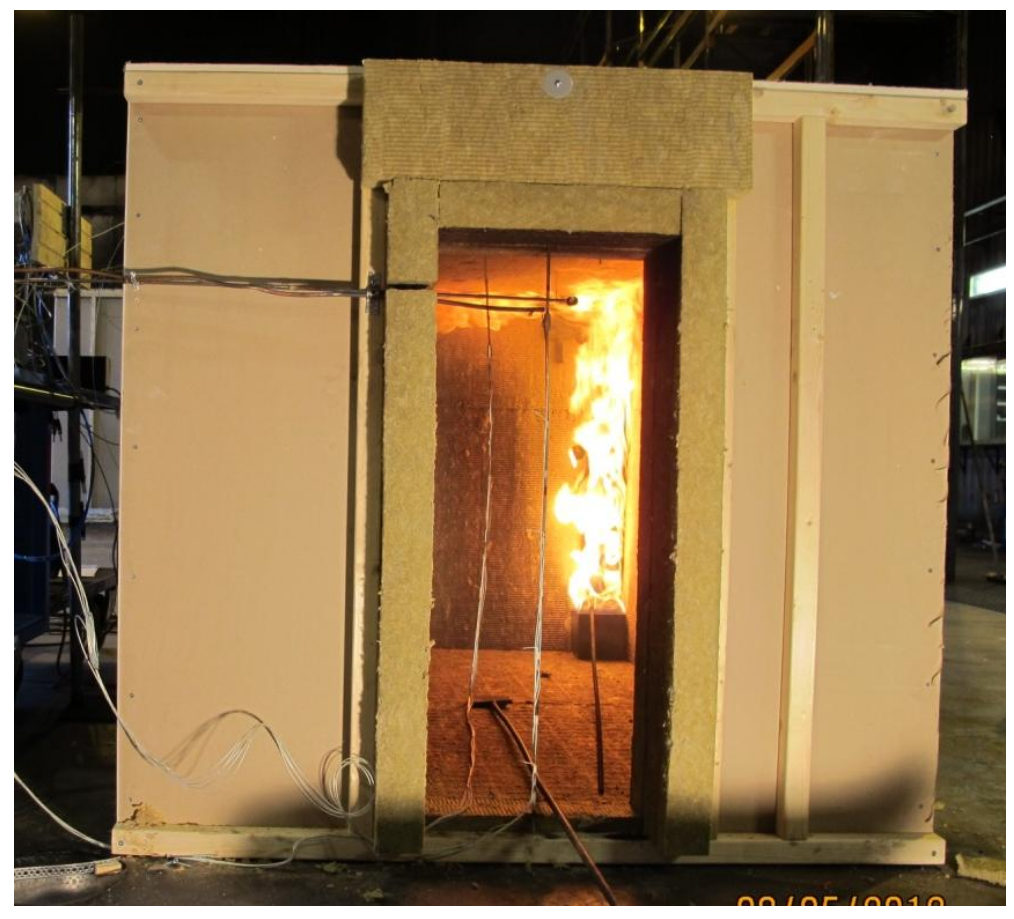

(b) Second stage 

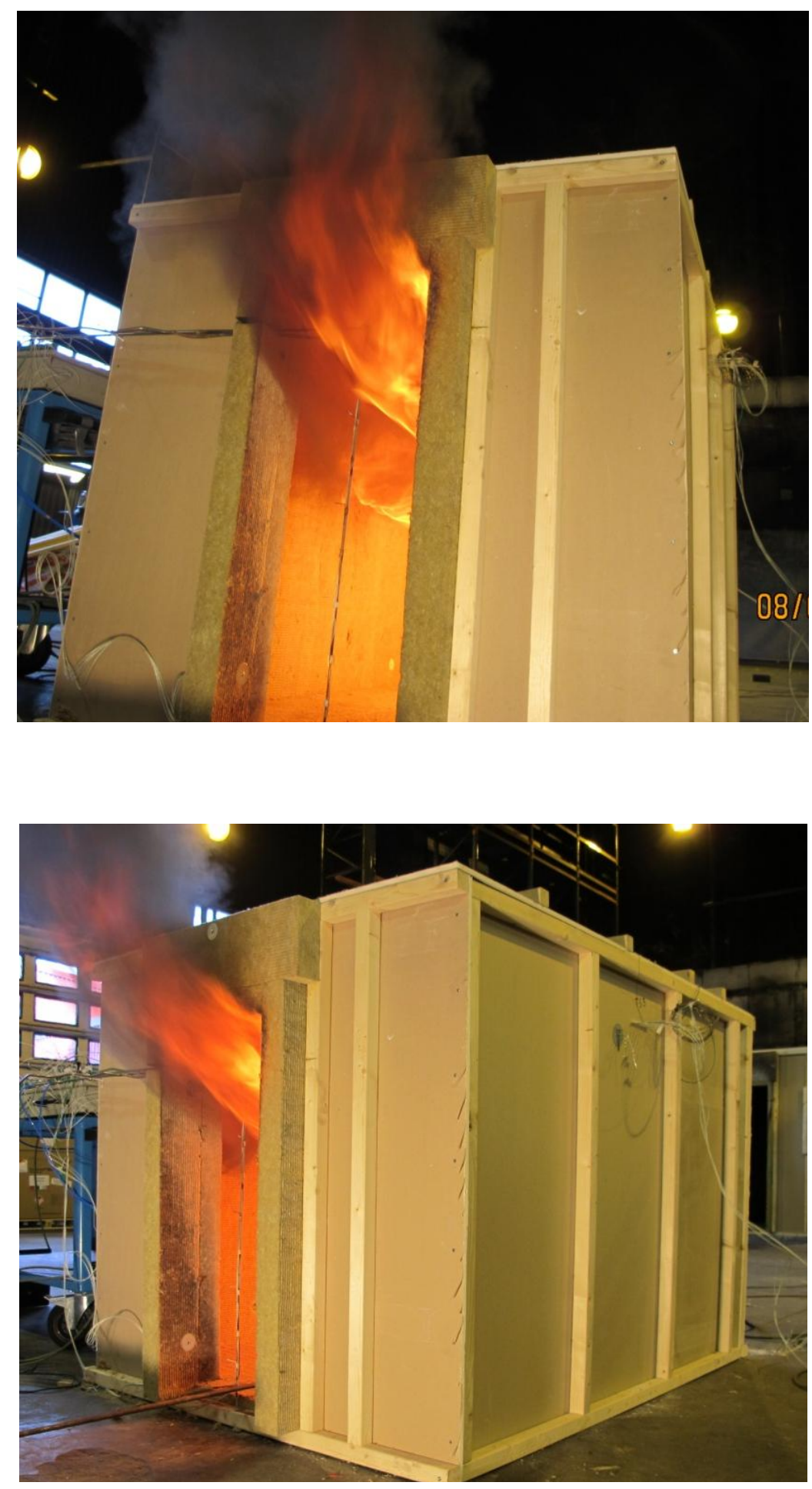

(c) Third stage

Figure C-5. Photos in medium scale test 5. 


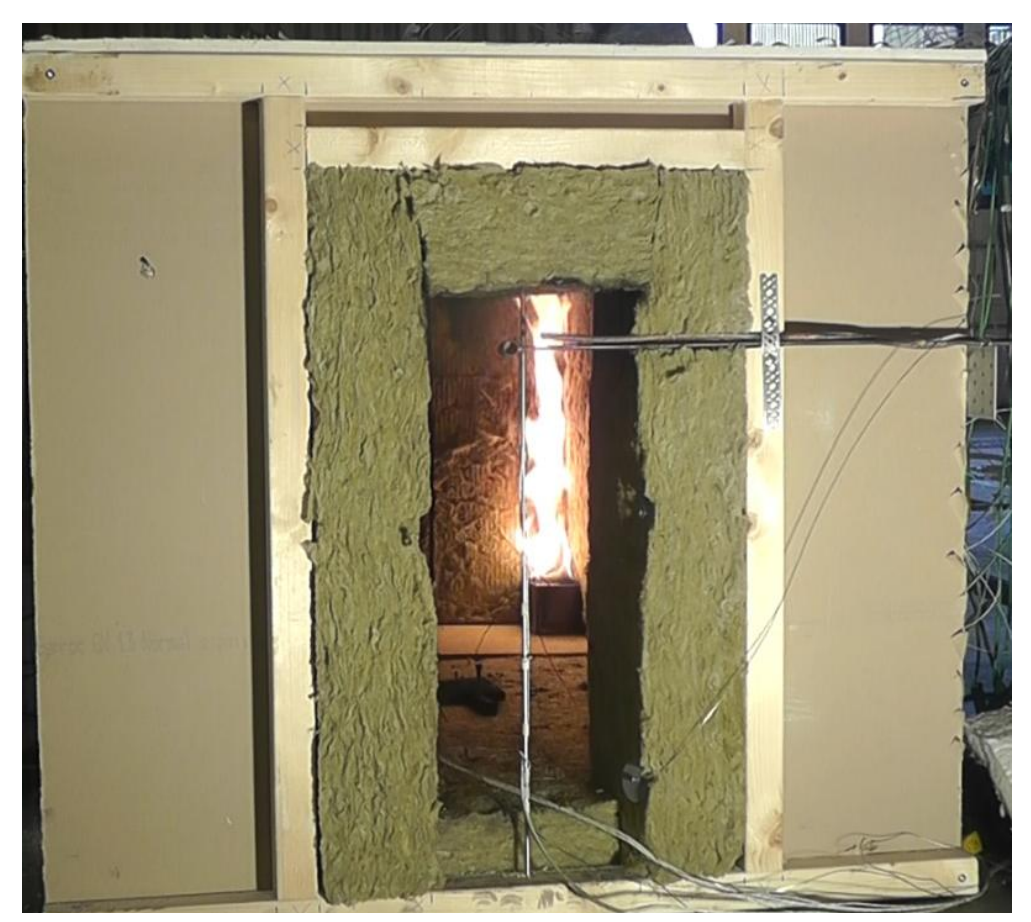

(a) First stage

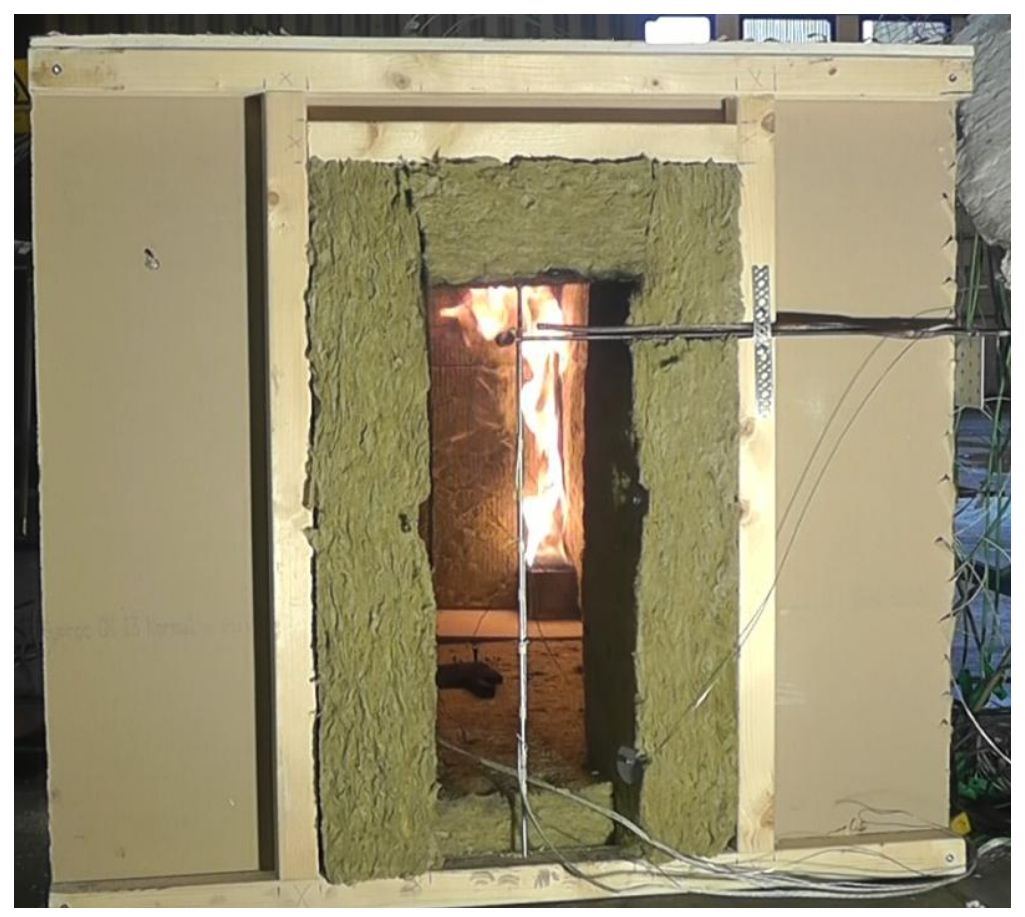

(b) Second stage 

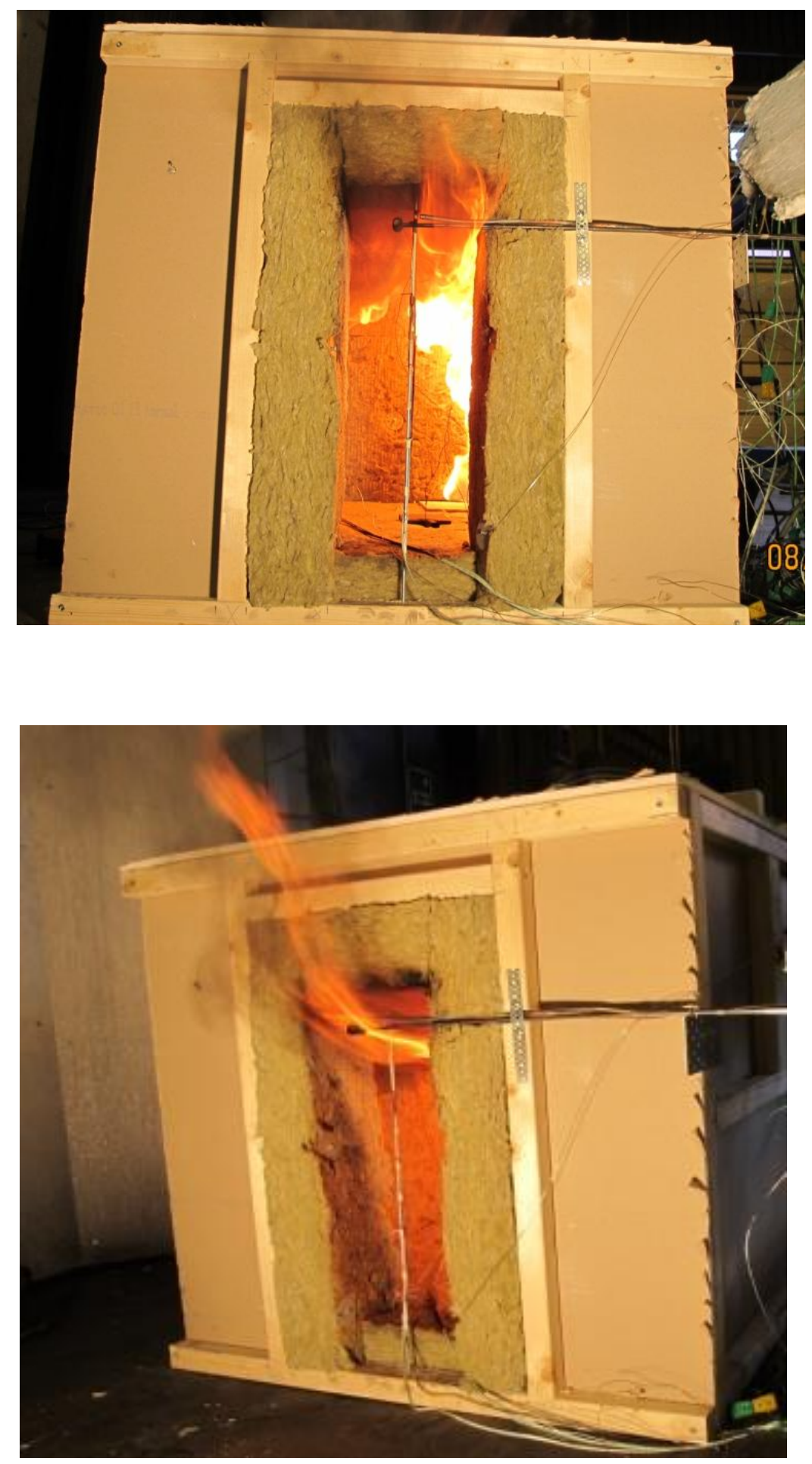

(c) Third stage

Figure C-6. Photos in small scale test 6. 


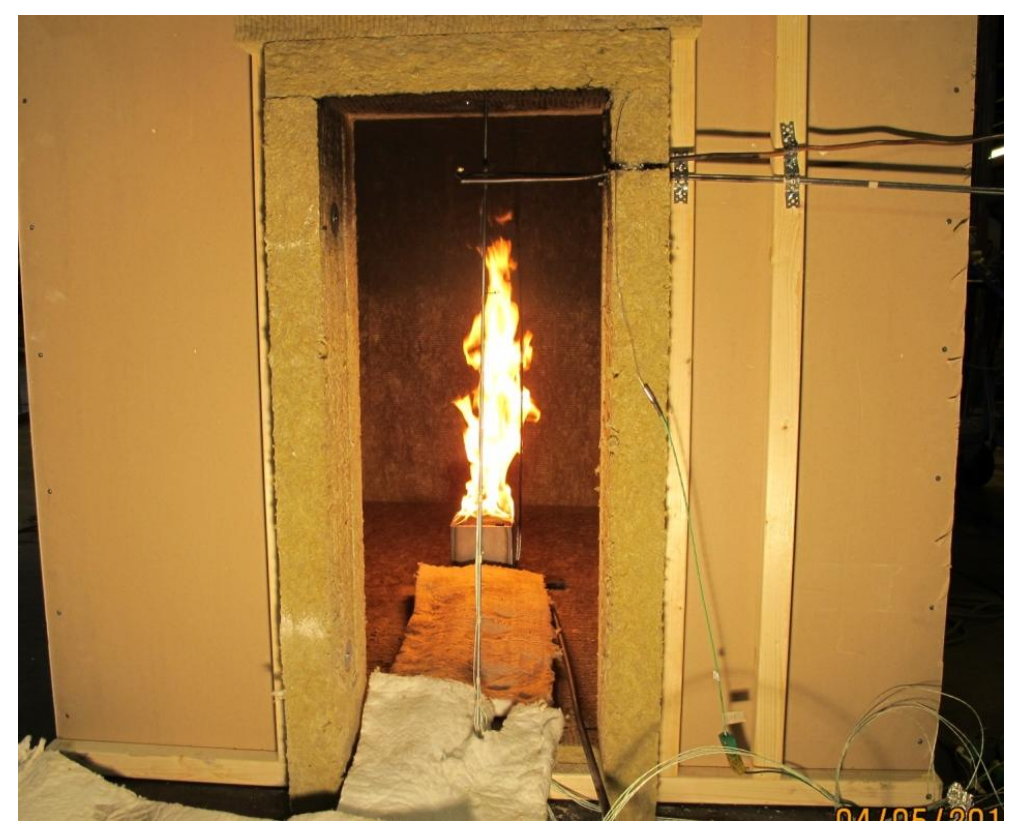

(a) First stage

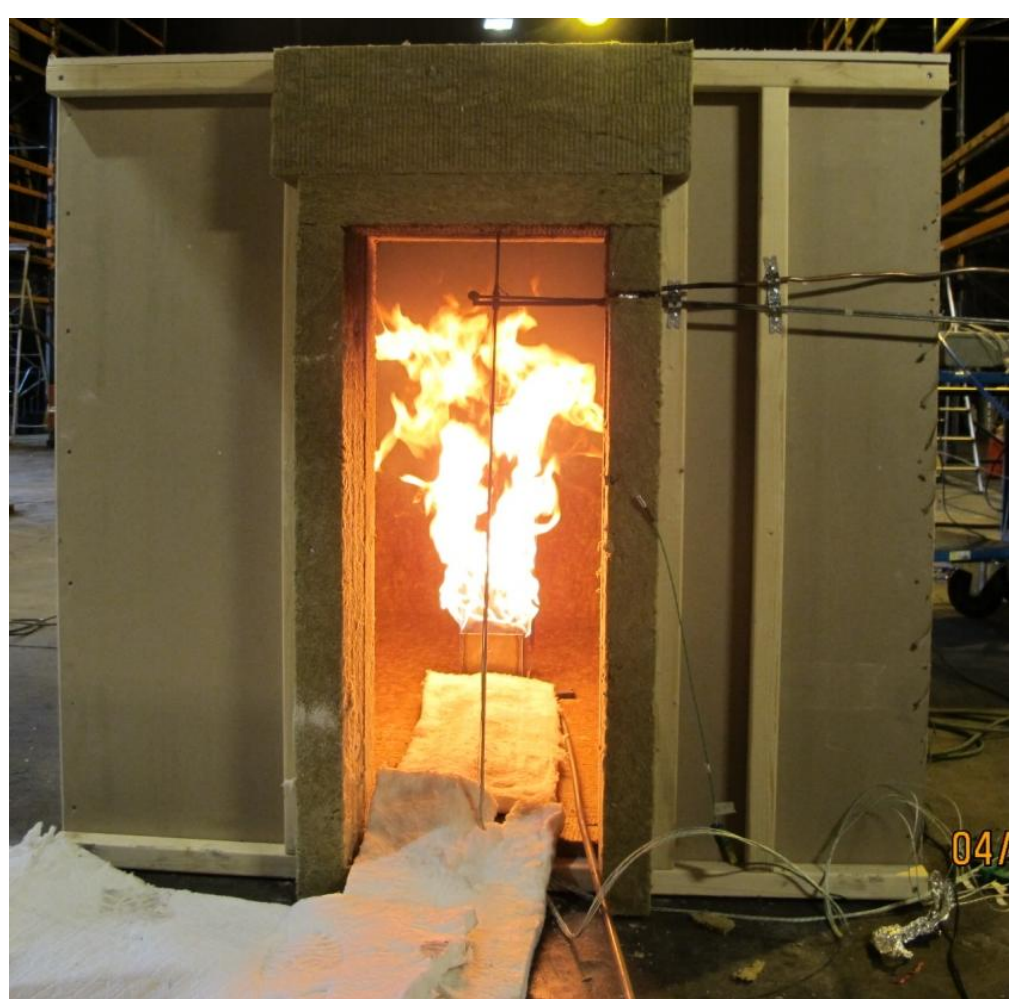

(b) Second stage 

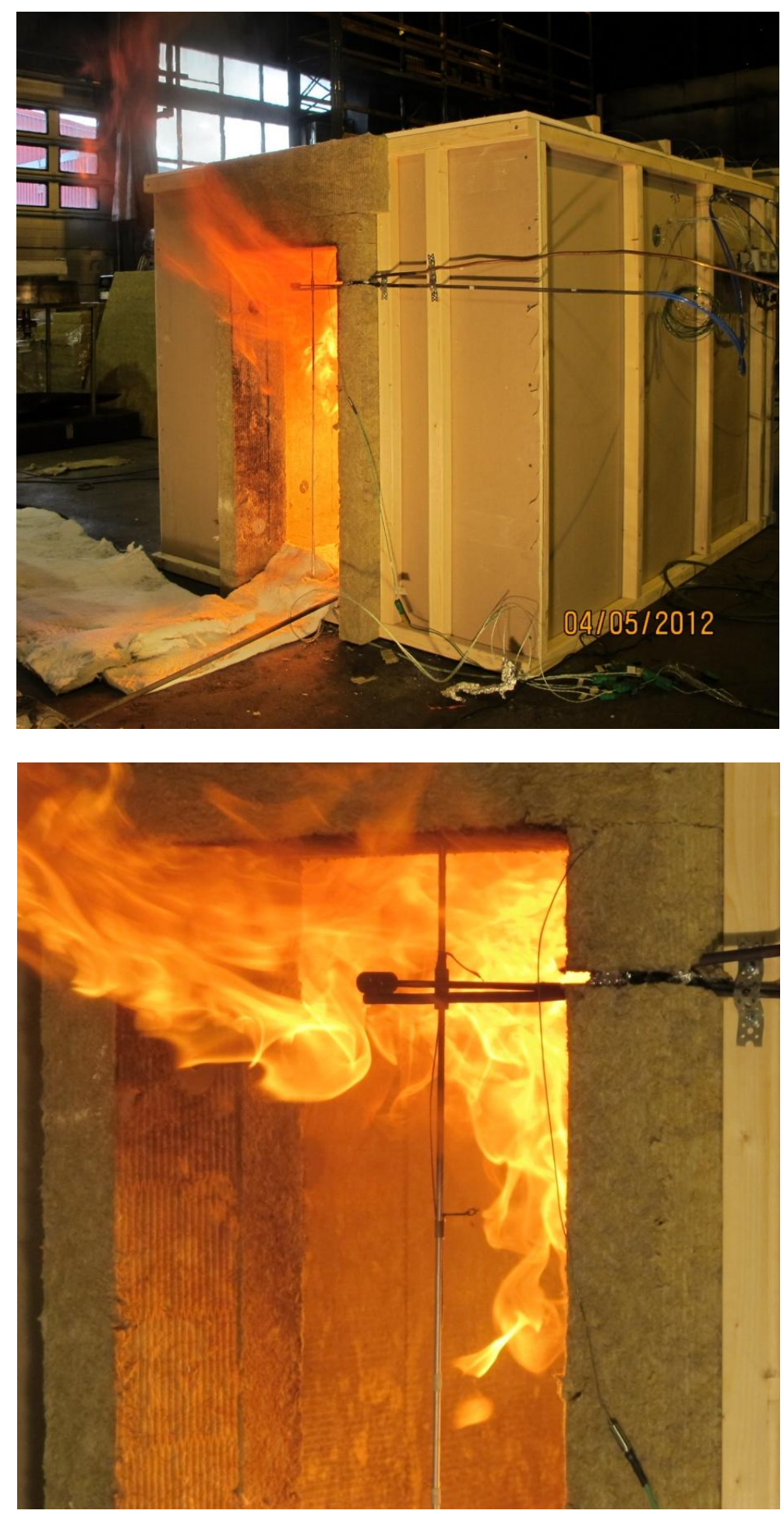

(c) Third stage

Figure C-7. Photos in medium scale test 7. 


\section{Appendix D - Test Results}

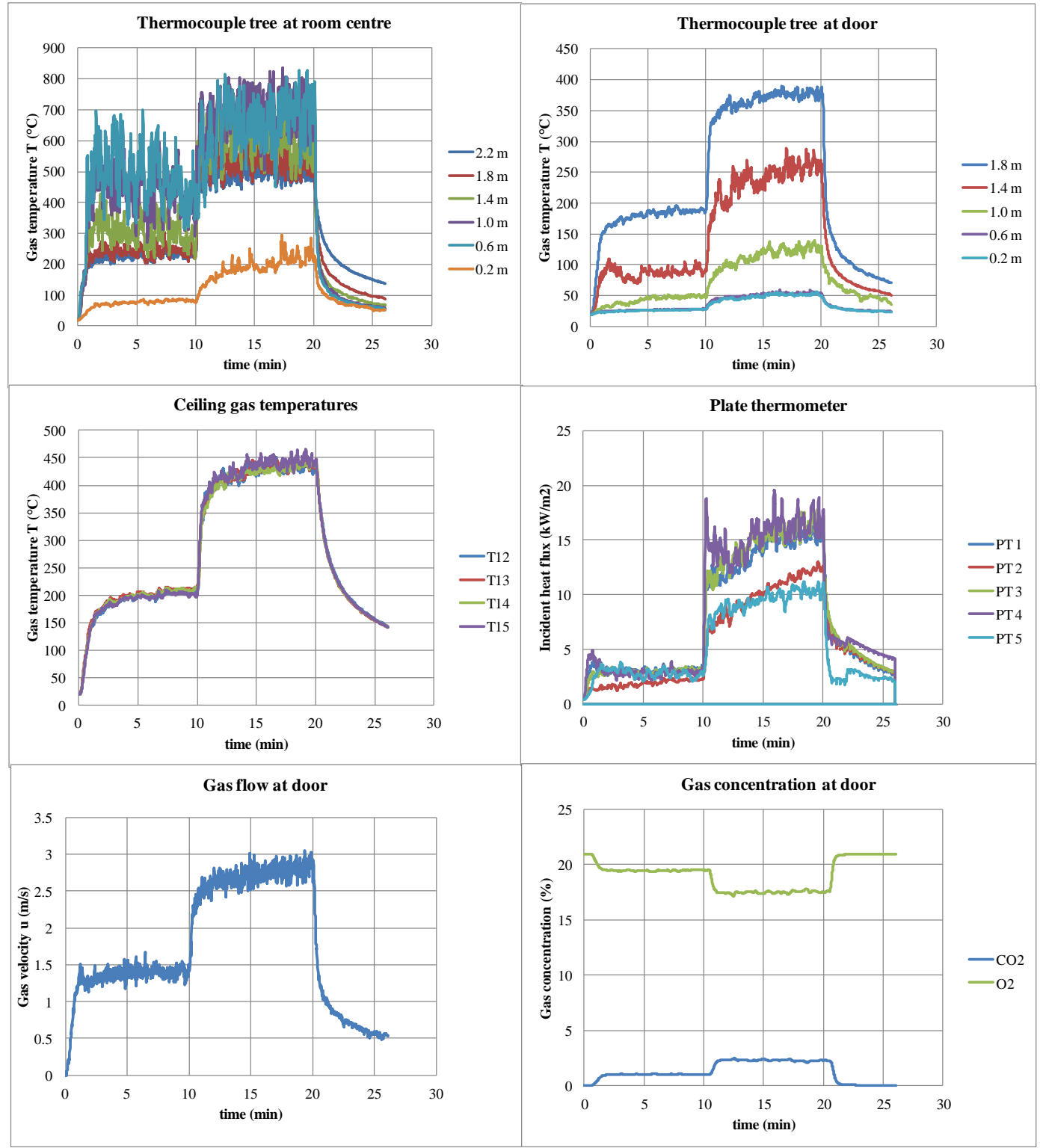

Figure D1 Gas temperatures, velocity, heat flux and concentration in Test 1. 


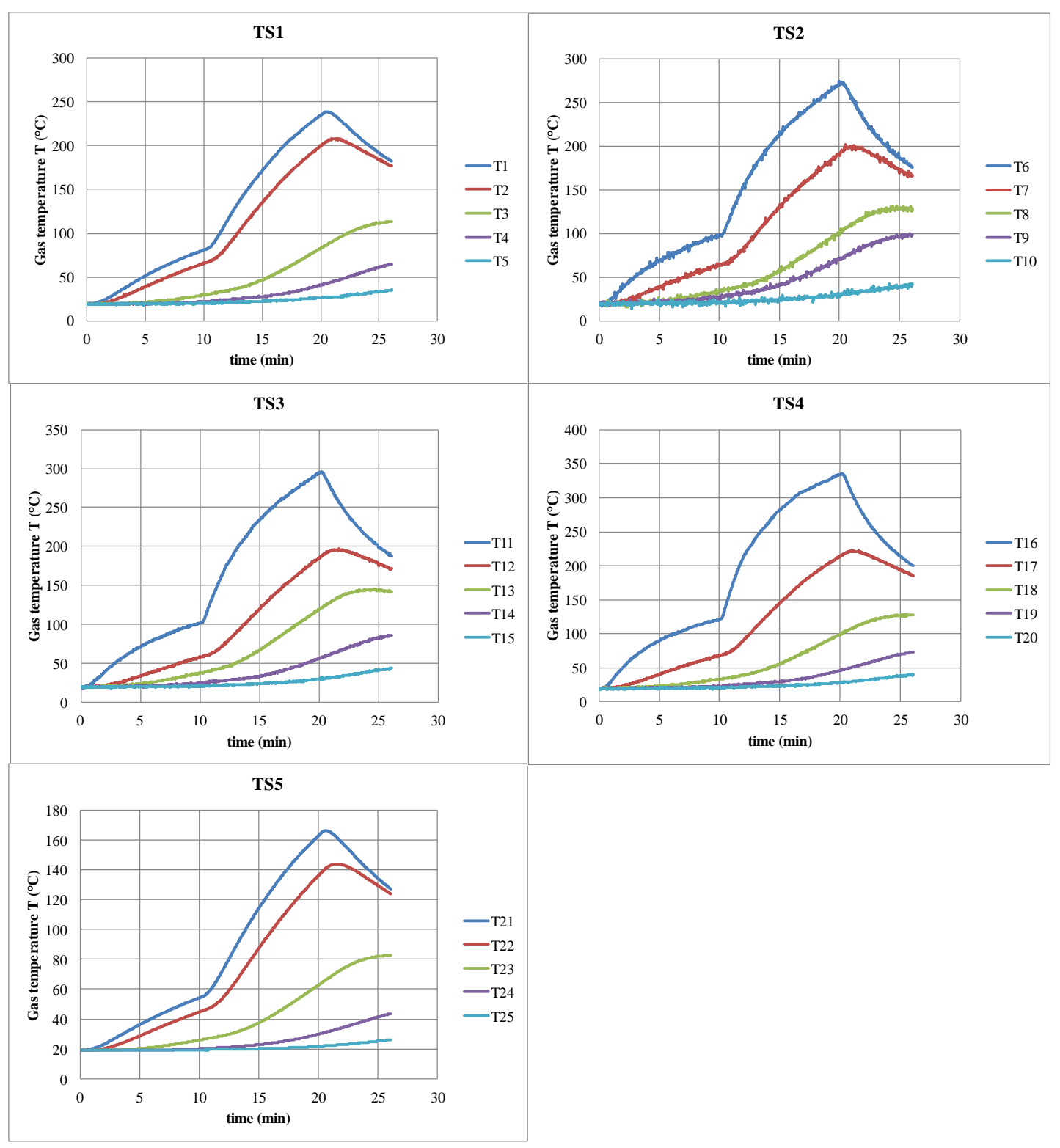

Figure D2 Measured temperatures inside the wall at positions TS1 to TS5 in Test 1. 

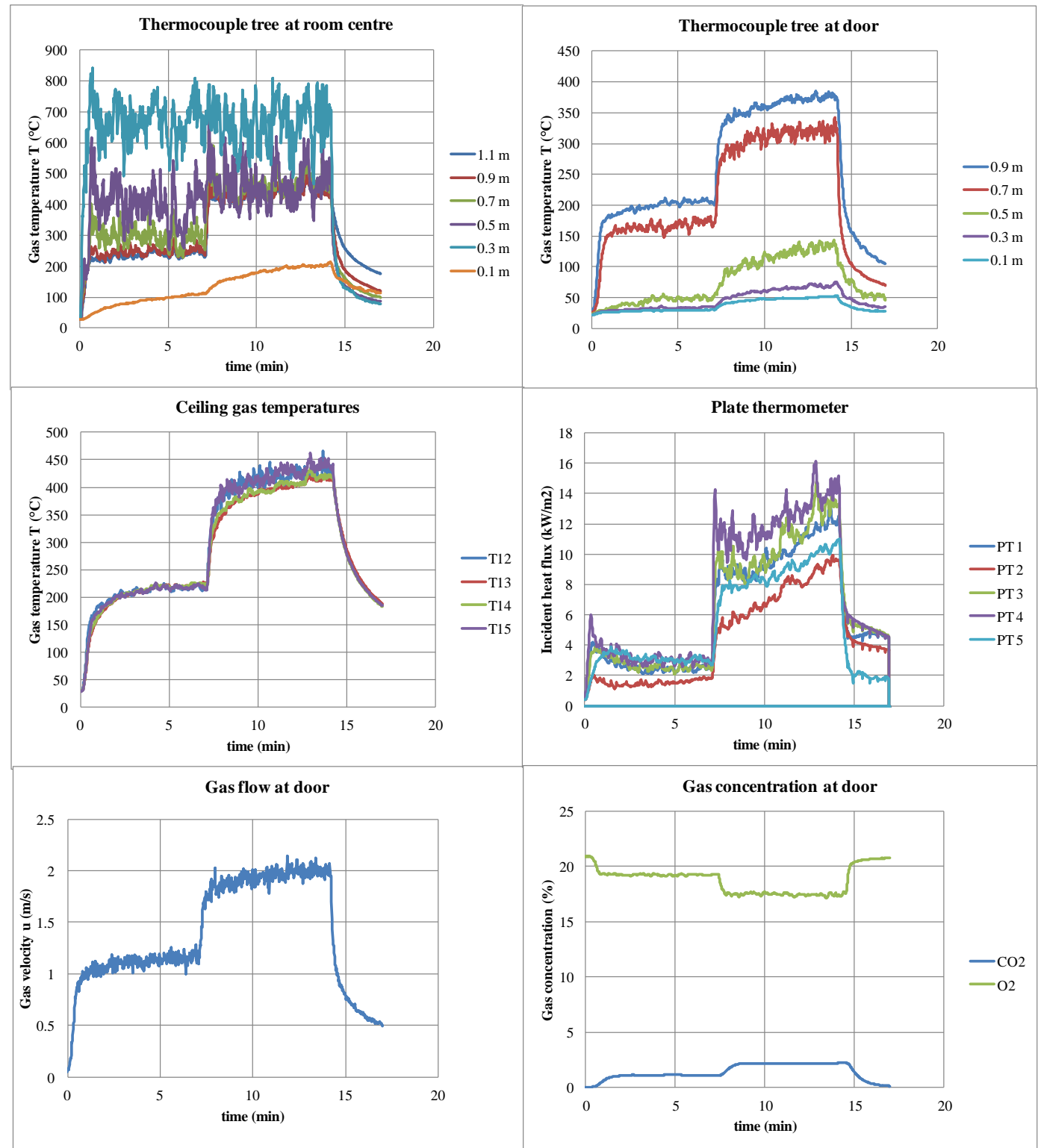

Figure D3 Gas temperatures, velocity, heat flux and concentration in Test 2. 


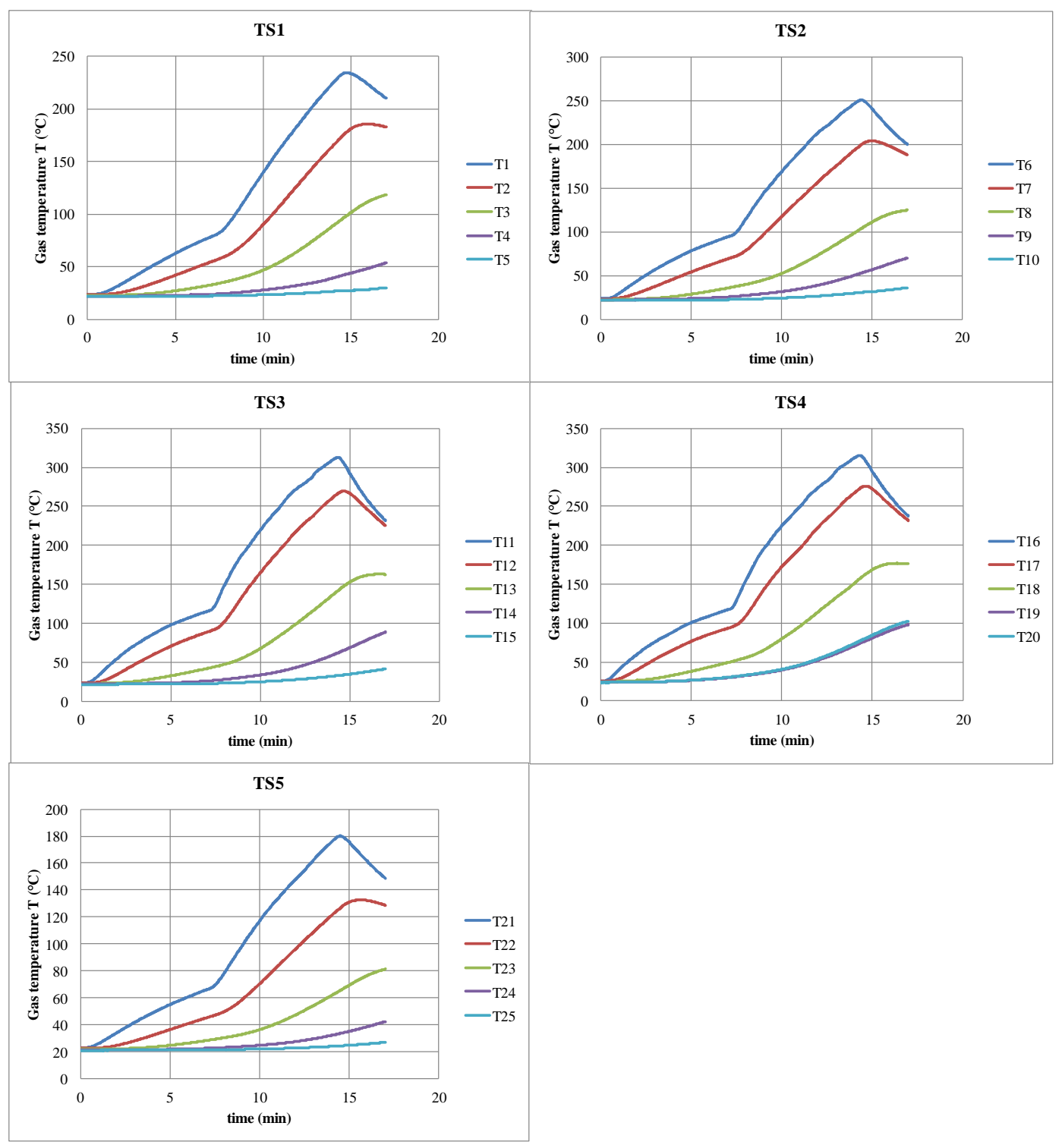

Figure D4 Measured temperatures inside the wall at positions TS1 to TS5 in Test 2. 

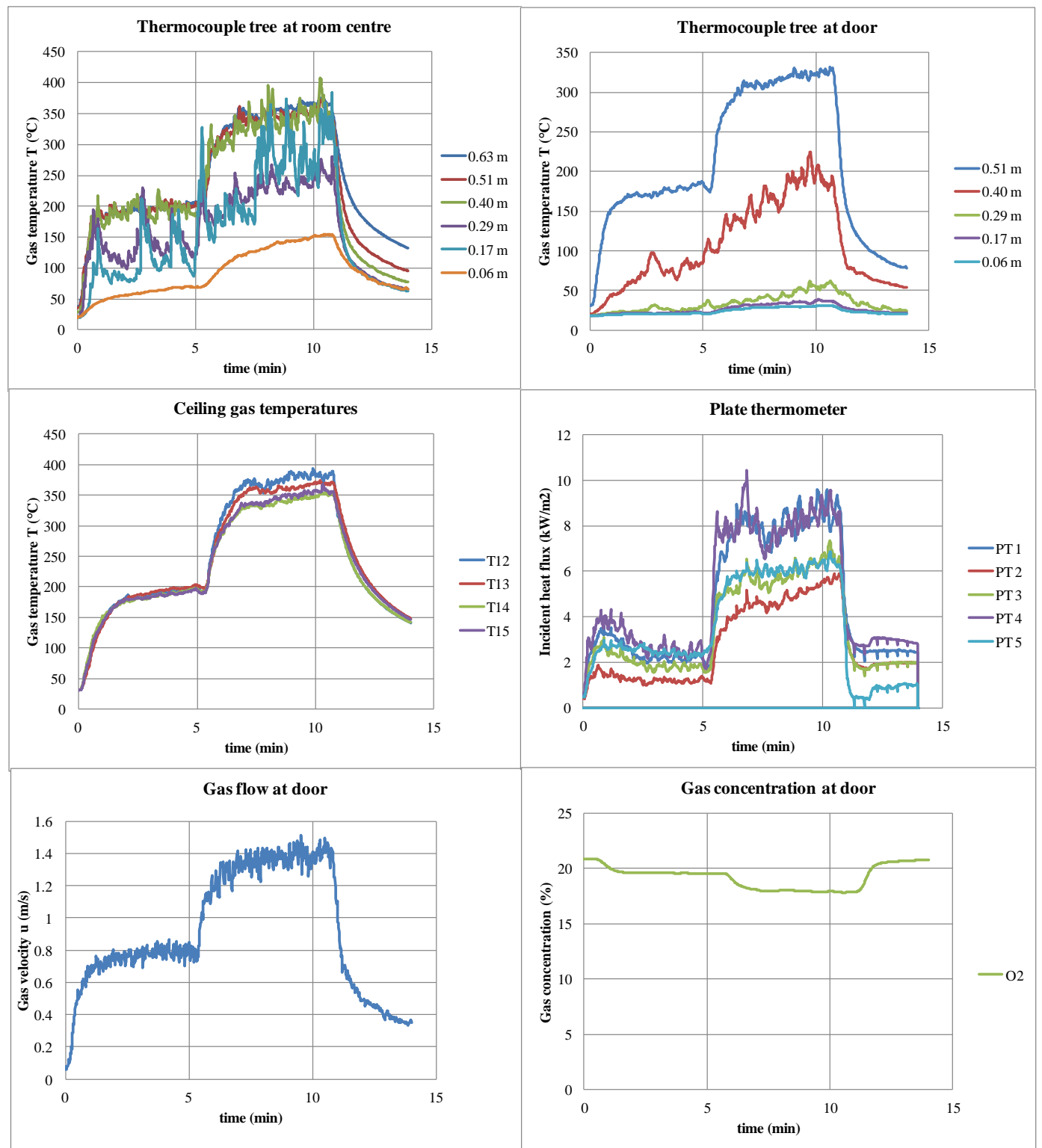

Figure D5 Gas temperatures, velocity, heat flux and concentration in Test 3. 


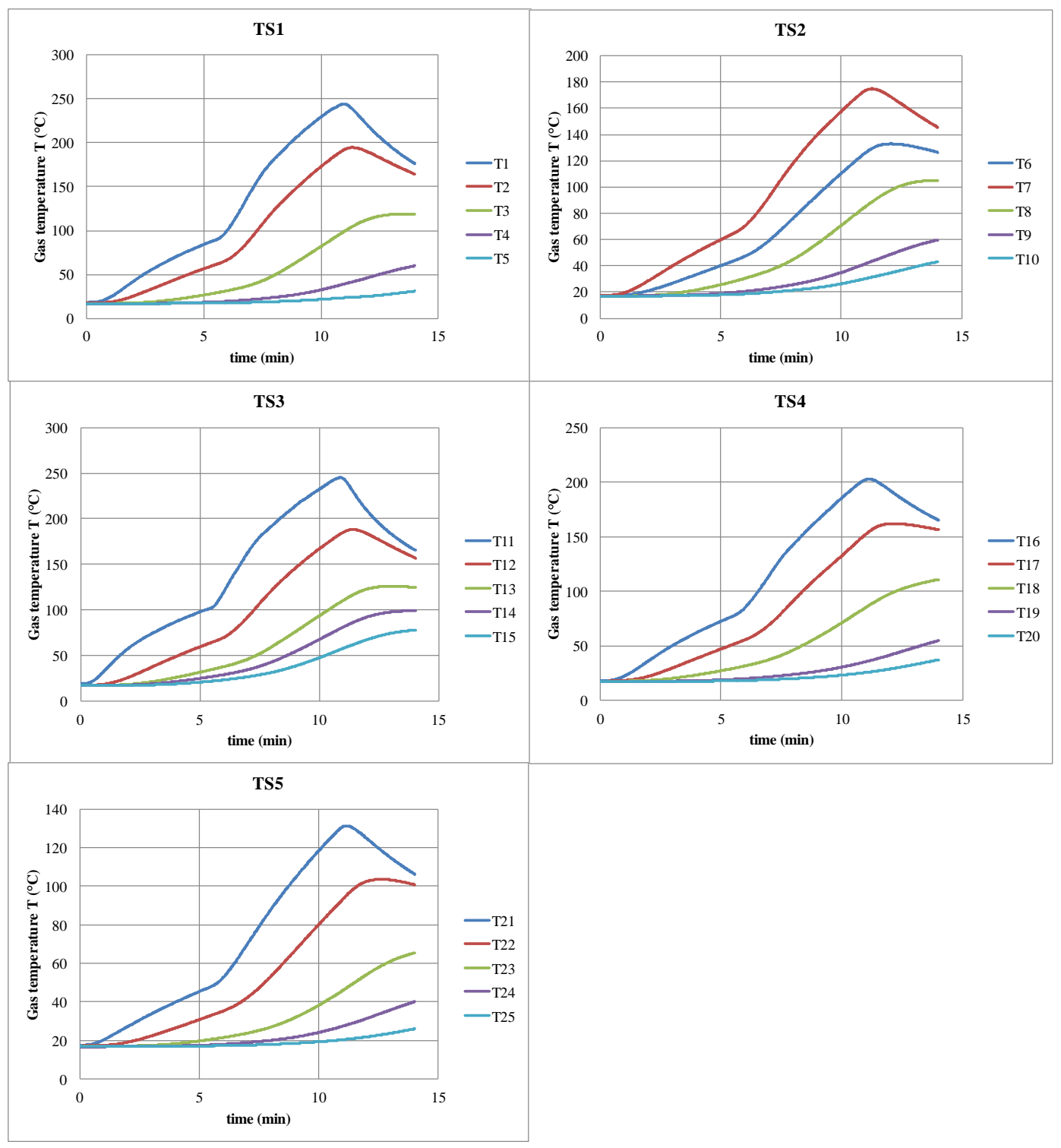

Figure D6 Measured temperatures inside the wall at positions TS1 to TS5 in Test 3. 

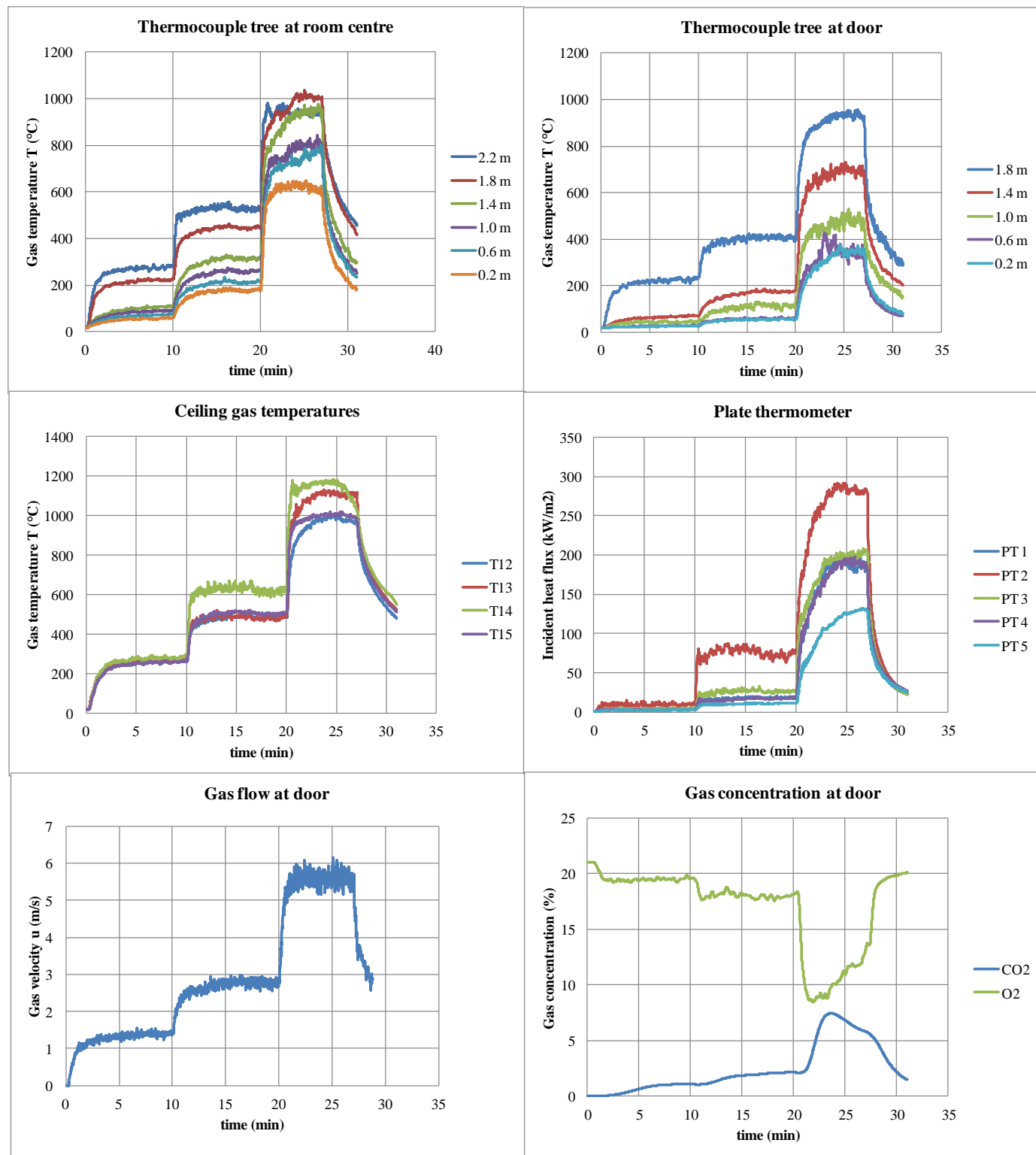

Figure D7 Gas temperatures, velocity, heat flux and concentration in Test 4. 

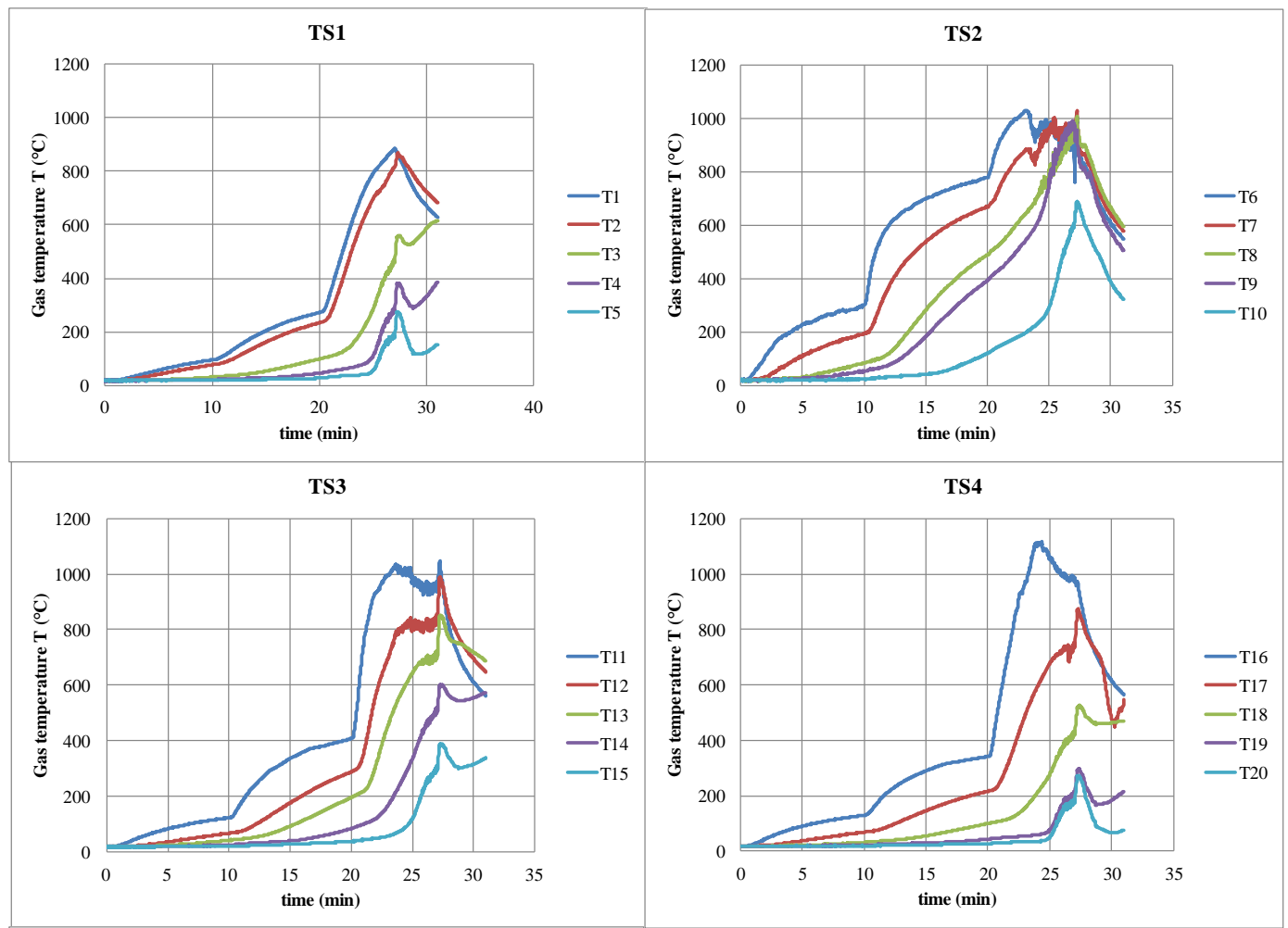

TS5

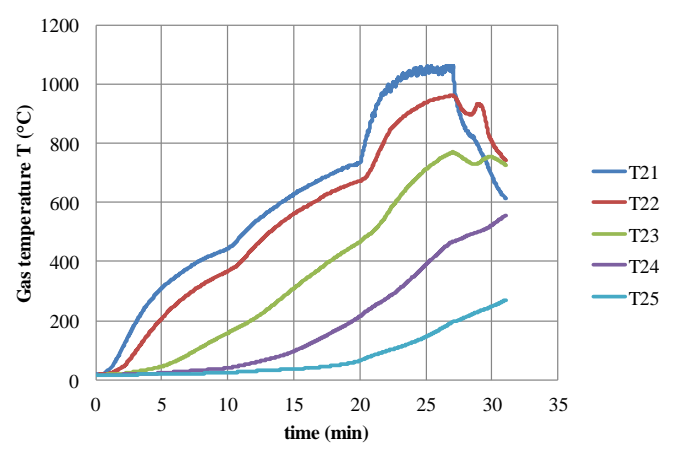

Figure D8 Measured temperatures inside the wall at positions TS1 to TS5 in Test 4. 

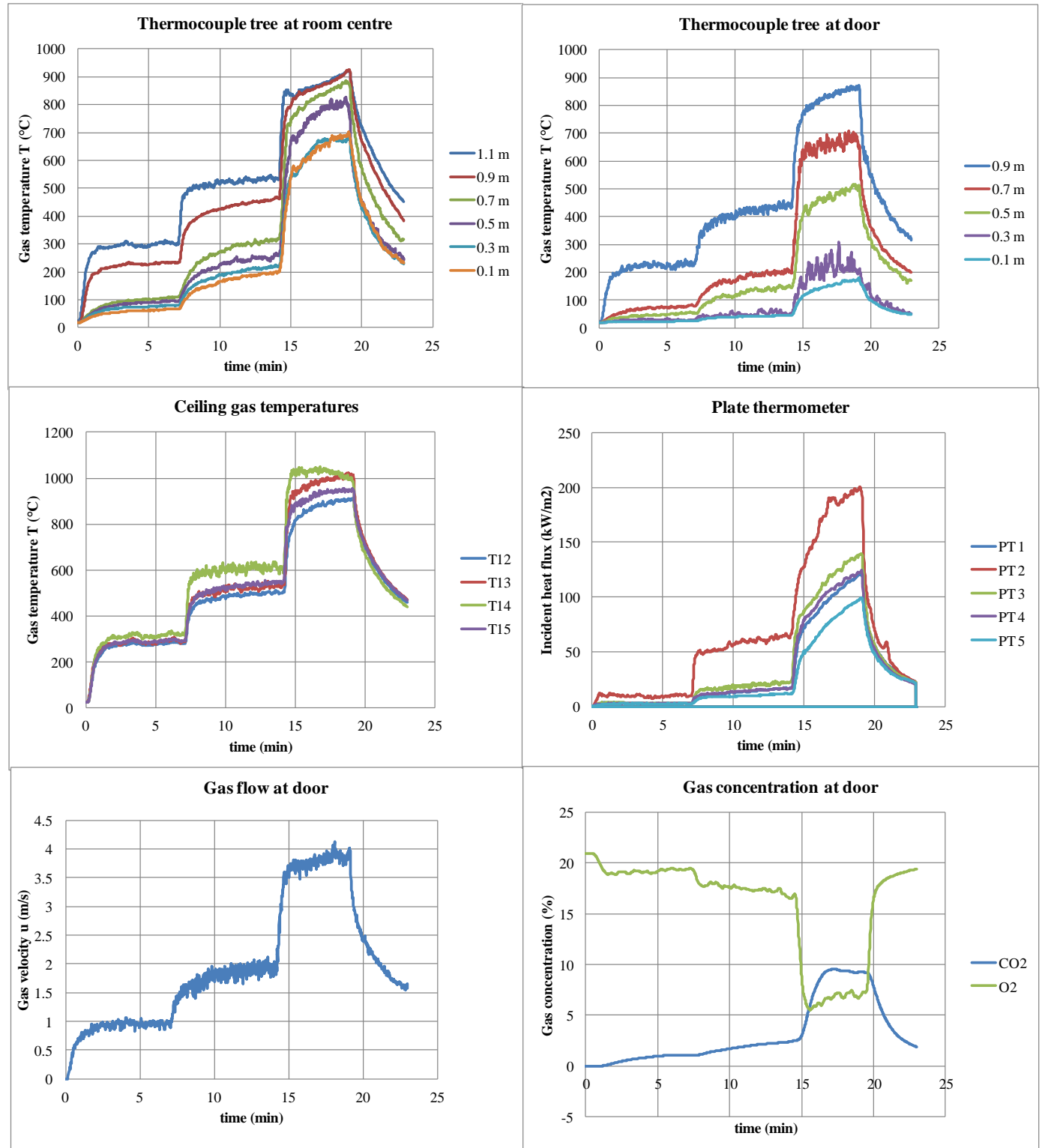

Figure D9 Gas temperatures, velocity, heat flux and concentration in Test 5. 

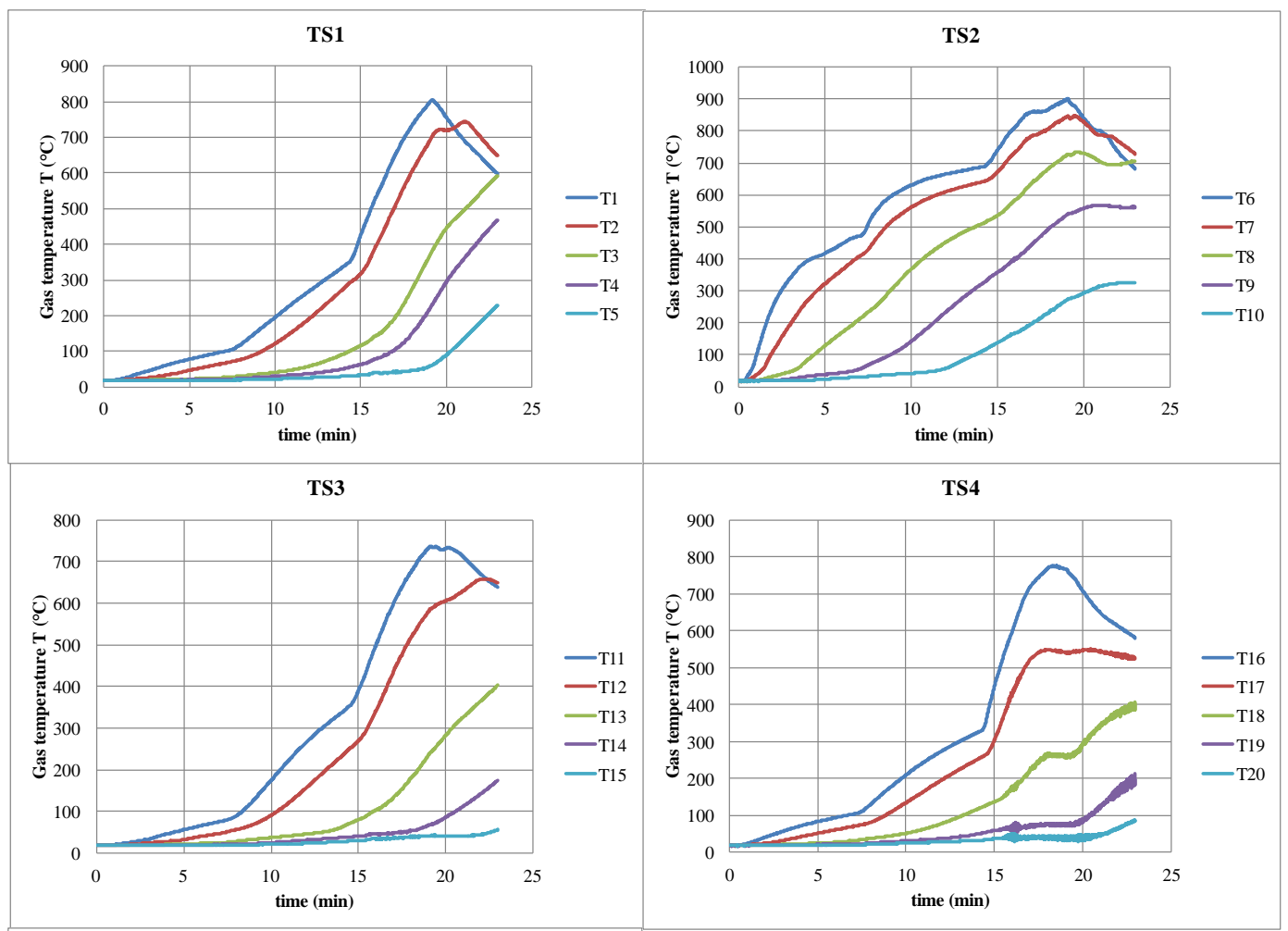

TS5

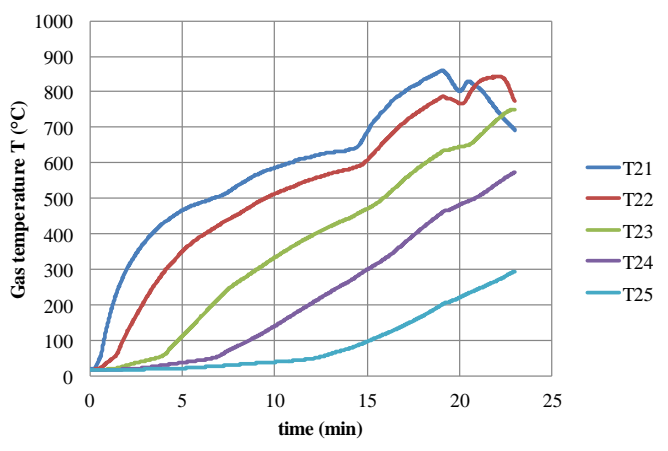

Figure D10 Measured temperatures inside the wall at positions TS1 to TS5 in Test 5. 

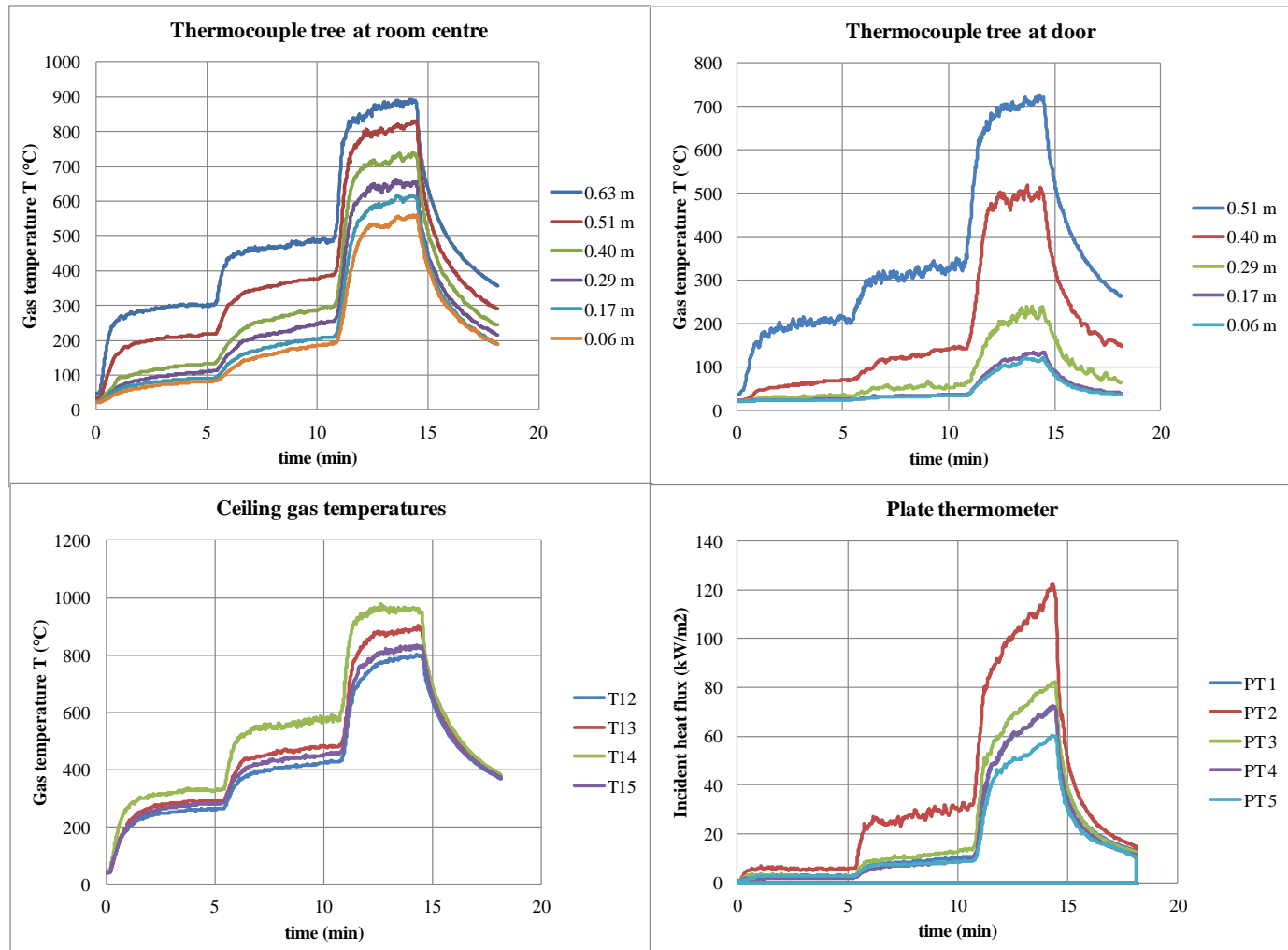

Plate thermometer
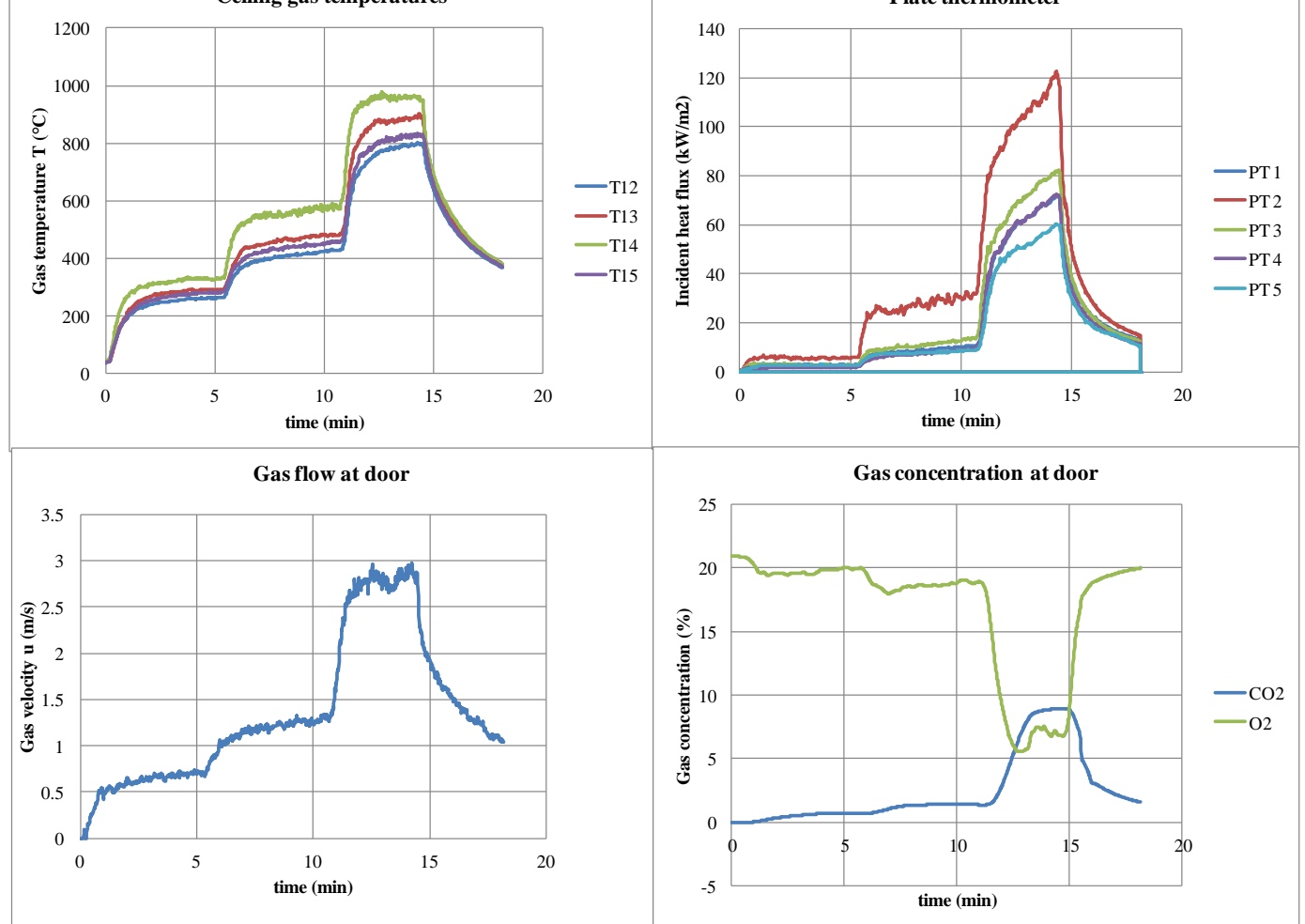

Figure D11 Gas temperatures, velocity, heat flux and concentration in Test 6. 


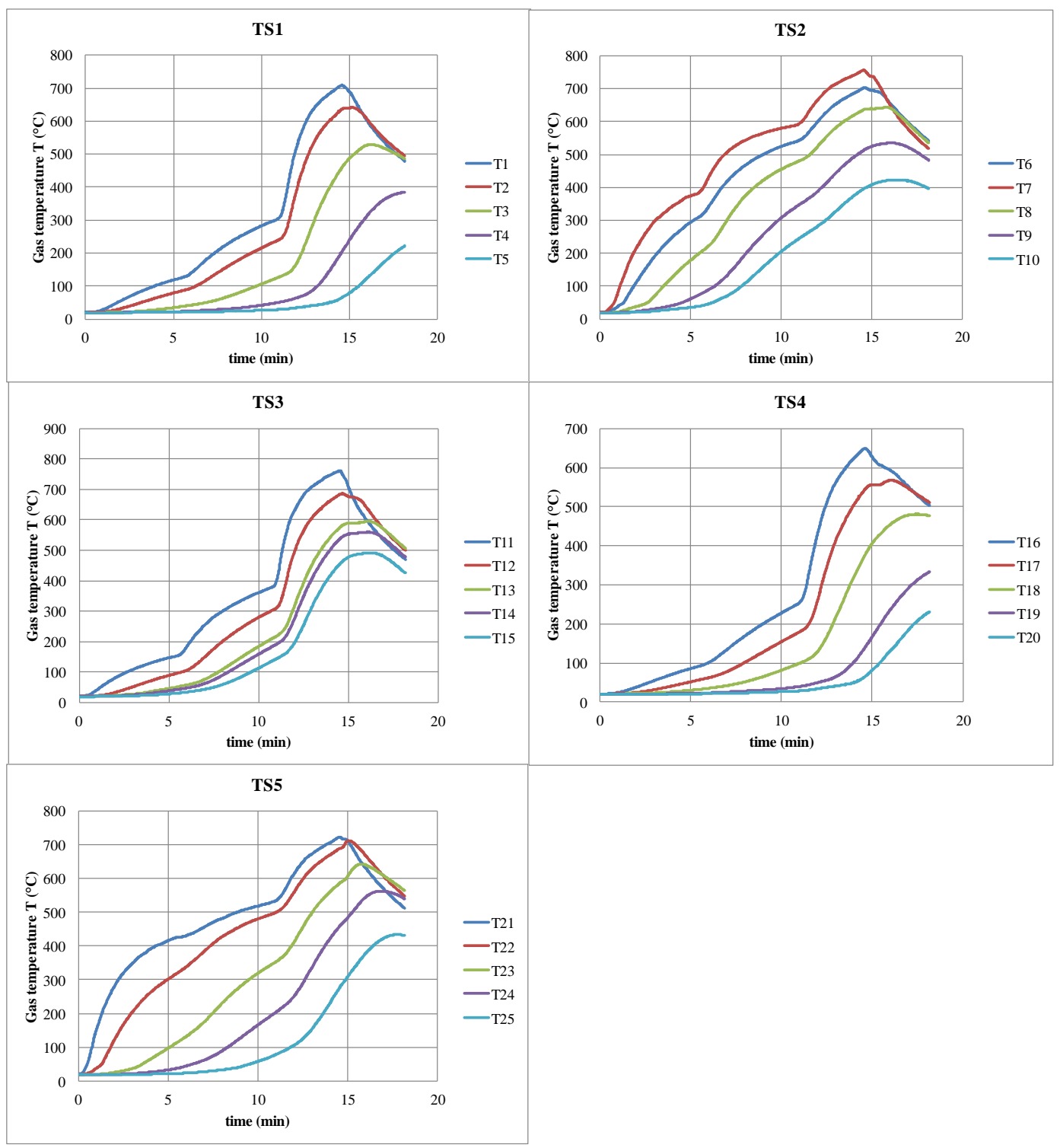

Figure D12 Measured temperatures inside the wall at positions TS1 to TS5 in Test 6. 

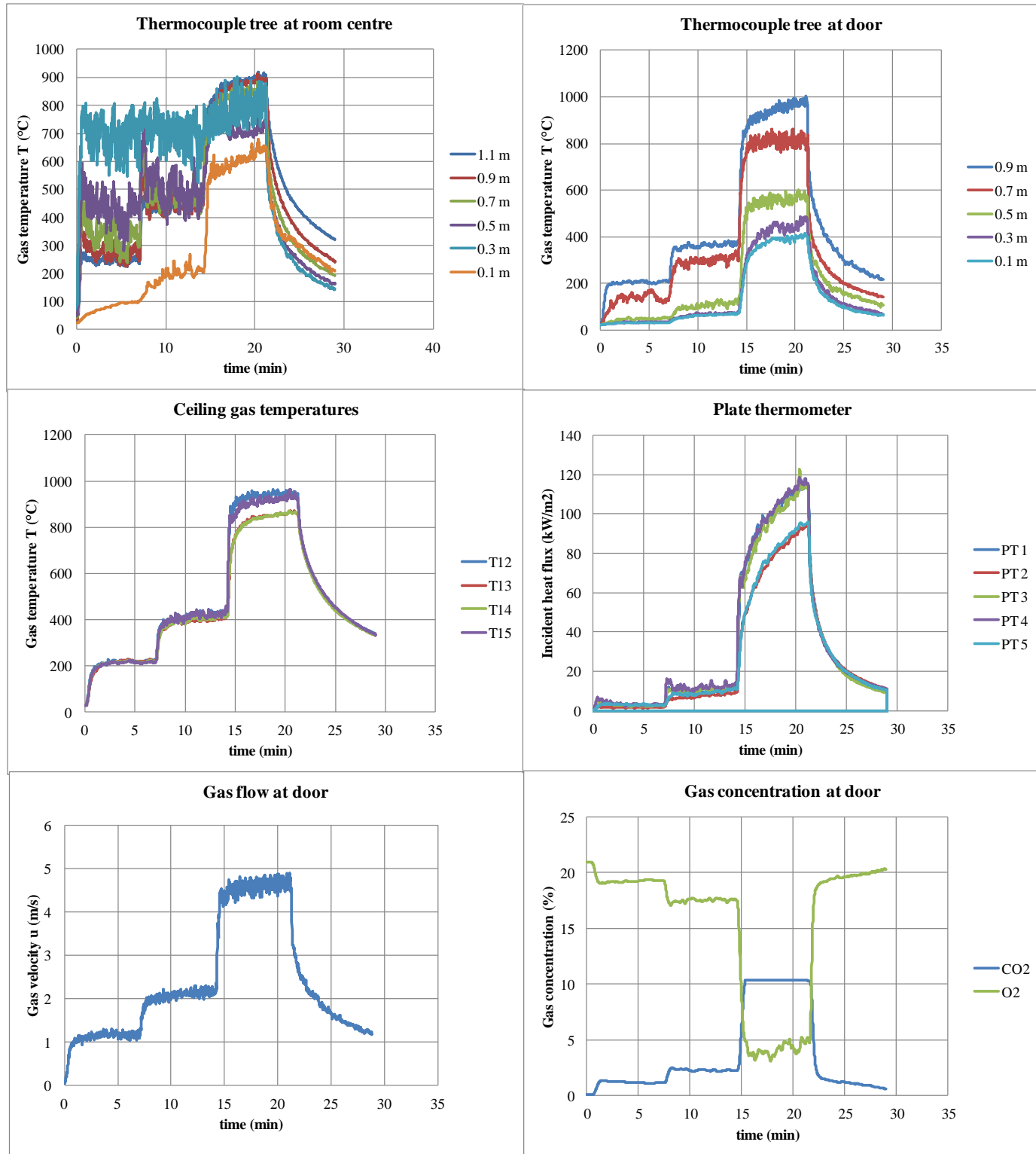

Figure D13 Gas temperatures, velocity, heat flux and concentration in Test 7. 

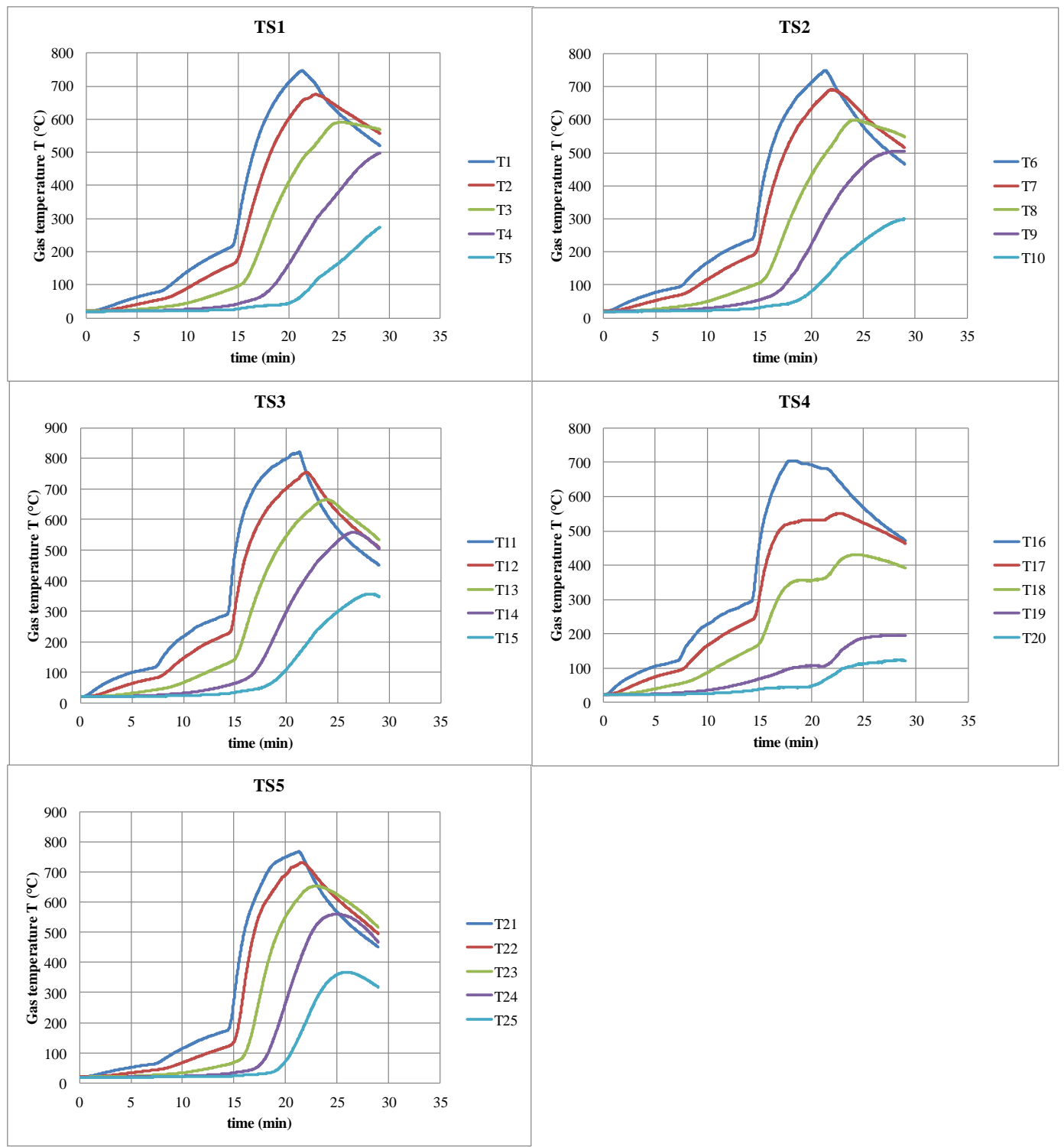

Figure D14 Measured temperatures inside the wall at positions TS1 to TS5 in Test 7. 


\section{SP Technical Research Institute of Sweden}

Our work is concentrated on innovation and the development of value-adding technology. Using Sweden's most extensive and advanced resources for technical evaluation, measurement technology, research and development, we make an important contribution to the competitiveness and sustainable development of industry. Research is carried out in close conjunction with universities and institutes of technology, to the benefit of a customer base of about 9000 organisations, ranging from start-up companies developing new technologies or new ideas to international groups.

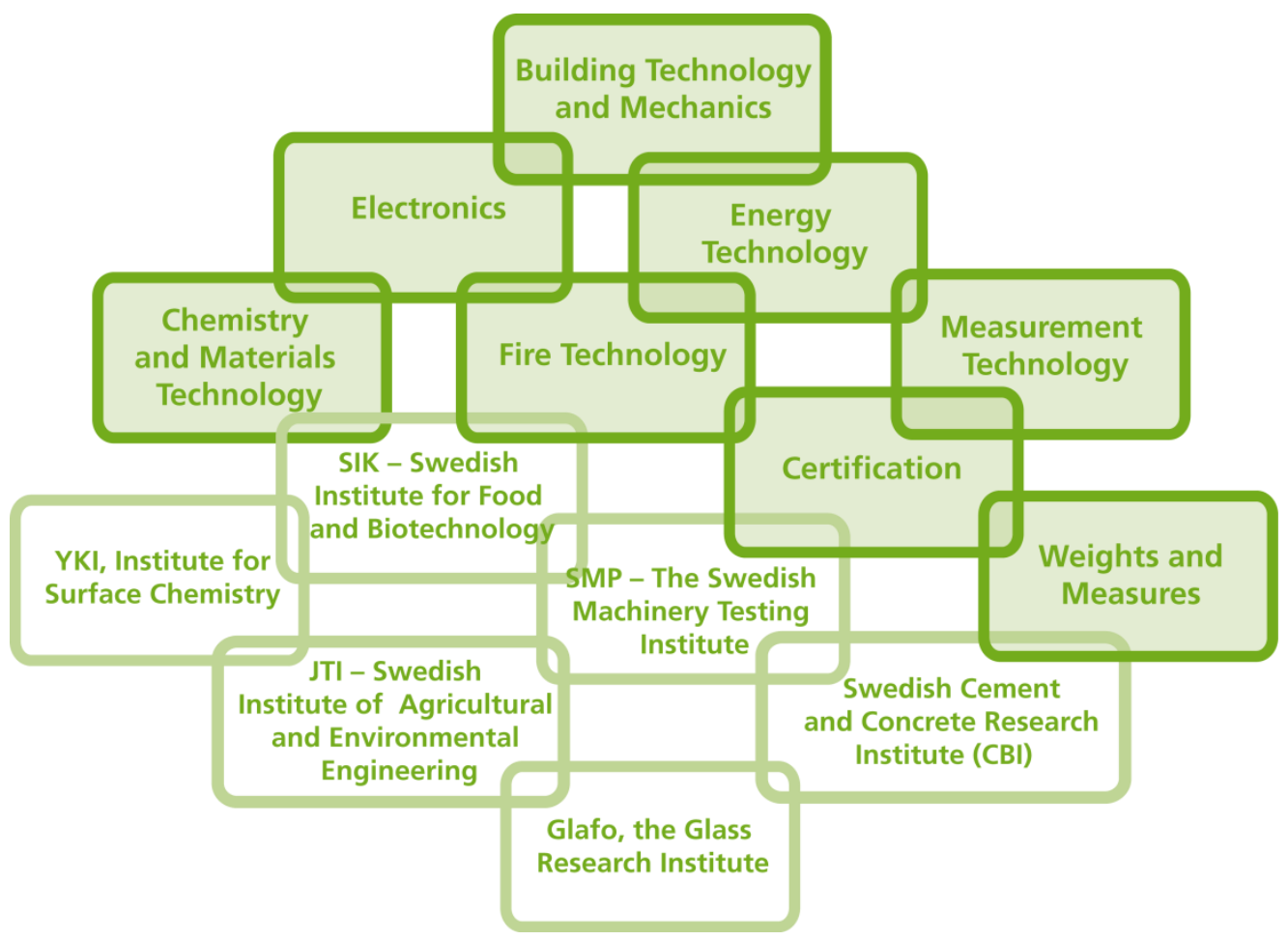

SP Technical Research Institute of Sweden 